RENAN CERQUEIRA AFONSO ALVES

Achieving efficient routing in constrained networks with unidirectional links through Software Defined Networking

São Paulo 
RENAN CERQUEIRA AFONSO ALVES

\section{Achieving efficient routing in constrained networks with unidirectional links through Software Defined Networking} versidade de São Paulo para obtenção do Título de Doutor em Ciências.

Orientadora: $\operatorname{Prof}^{a} \operatorname{Dr}^{a}$ Cíntia Borges Margi

Versão Corrigida

São Paulo

2020 
Autorizo a reprodução e divulgação total ou parcial deste trabalho, por qualquer meio convencional ou eletrônico, para fins de estudo e pesquisa, desde que citada a fonte.

Este exemplar foi revisado e corrigido em relação à versão original, sob responsabilidade única do autor e com a anuência de seu orientador.

São Paulo, de de

Assinatura do autor:

Assinatura do orientador:

\section{Catalogação-na-publicação}

\section{Alves, Renan Cerqueira Afonso}

Achieving efficient routing in constrained networks with unidirectional links through Software Defined Networking / R. C. A. Alves -- versão corr. -São Paulo, 2020.

$119 \mathrm{p}$.

Tese (Doutorado) - Escola Politécnica da Universidade de São Paulo. Departamento de Engenharia de Computação e Sistemas Digitais.

1.Protocolos de comunicação 2.Wireless 3.Internet das coisas 4.Sistemas distribuídos I.Universidade de São Paulo. Escola Politécnica. Departamento de Engenharia de Computação e Sistemas Digitais II.t. 


\section{Agradecimentos}

Aos meus amigos Jany, Pedro e Yasmine, pela companhia diária, pequenas alegrias do dia-a-dia e amor incondicional.

À minha família que, apesar da distância, sempre me apoiou.

Aos amigos que fiz no período de doutorado sanduíche, Billy, Ramon, Dustin, Eric, Belma, Jorik, para citar alguns, pelo acolhimento e todos os momentos de diversão.

À Maria José, pelos valiosos conselhos.

Aos colegas de laboratório, pelas conversas, trocas e incentivo.

À Cíntia Margi, minha orientadora, pela longa parceria, conselhos e paciência.

Ao Fernando Kuipers, por ser meu orientador durante estágio de pesquisa no exterior, oportunidade que me proporcionou grande crescimento pessoal e maturidade como pesquisador.

A FAPESP, pelo apoio financeiro, processos n n 2016/21088-1 e 2018/11295-5, Fundação de Amparo à Pesquisa do Estado de São Paulo (FAPESP).

Ao CNPq, pelo apoio financeiro, processo $n^{o}$ 155372/2016-5, Conselho Nacional de Desenvolvimento Científico e Tecnológico.

O presente trabalho foi realizado com apoio da Coordenação de Aperfeiçoamento de Pessoal de Nível Superior - Brasil (CAPES) - Código de Financiamento 001. 
不怕慢, 就怕停 Be not afraid of growing slowly, be afraid of standing still. Chinese saying 


\section{Resumo}

ALVES, Renan Cerqueira Afonso. Achieving efficient routing in constrained networks with unidirectional links through Software Defined Networking. 2020. Tese (Doutorado) Escola Politécnica, Universidade de São Paulo, São Paulo, 2020.

As Redes de Sensores Sem Fio são compostas por dispositivos restritos, sujeitos a baixa oferta de energia, capacidade de processamento limitada e quantidade de memória reduzida. O enlace de comunicação entre dois dispositivos pode se tornar unidirecional devido a diferenças de potência de transmissão, antenas não isotrópicas ou heterogeneidade de dispositivos; contudo, o uso destes enlaces pode reduzir o tamanho de rotas de comunicação, economizando recursos. O objetivo desta pesquisa é projetar protocolos que permitam usar estes enlaces, atendendo aos requisitos impostos pela limitação de recursos dos dispositivos. O paradigma de Redes Definidas por Software foi utilizado para solucionar este desafio, uma vez que o controle centralizado deste paradigma facilita o cálculo de rotas com enlaces unidirecionais, de forma genérica e sem recorrer a estratégias de inundação de pacotes. Para tanto, foram projetados algortimos de descoberta de vizinhos e descoberta de controlador necessários para o funcionamento de Redes de Sensores Definidas por Software. Primeiramente, algoritmos simples foram projetados para demonstrar a viabilidade da proposta; em seguida, estes algortimos foram aprimorados para proporcionar escalabilidade. Experimentos com dispositivos foram executados para verificar a ocorrência de enlaces unidirecionais, enquanto que os algoritmos propostos foram implementados e testados em um ambiente simulado/emulado. Os testes foram realizados em redes de 16 até 100 nós, dispostos em grade ou aleatoriamente. Cada cenário foi variado de quatro formas: todos os enlaces bidirecionais, enlaces unidirecionais selecionados aletoriamente, dispositivos com alcance aumentado e controlador da rede com alcance para todos os dispositivos da rede. Como base de comparação, foi utilizado o algoritmo de descoberta baseado no protocolo Collect, comumente utilizado em Redes de Sensores Definidas por Software. O uso de enlaces unidirecionais proporcionou taxa de entrega de pacotes de ao menos 90\%, enquanto que o protocolo Collect apresentou taxa de entrega inferior a 50\%, considerando os cenários com enlaces unidirecionais contemplados pelos experimentos executados. Por fim, um algoritmo de ciclo de trabalho de rádio assíncrono foi projetado, tanto quanto sabemos, o primeiro a oferecer suporte a enlaces unidirecionais. A análise dos experimentos contemplando ciclo de trabalho de rádio revelou que o uso de enlaces unidirecionais traz benefícios se os enlaces forem de longo alcance.

Keywords: Protocolos de comunicação. Wireless. Internet das coisas. Sistemas distribuídos. 


\section{Abstract}

ALVES, Renan Cerqueira Afonso. Achieving efficient routing in constrained networks with unidirectional links through Software Defined Networking. 2020. Tese (Doutorado) Escola Politécnica, Universidade de São Paulo, São Paulo, 2020.

Wireless Sensor Networks are composed of constrained devices subjected to low energy budget, limited processing capability, and reduced memory availability. A wireless communication link between two devices may become unidirectional due to transmission power disparity, non-isotropic antennas, or device heterogeneity; using such links holds the potential to shorten communication routes, saving resources. Our objective is to design a set of protocols that enable routing in a network with unidirectional links, while attending the requirements imposed by the limited resources available. We used the Software Defined Networking paradigm to solve this challenge, as the centralized control enables calculating routes with unidirectional links in a general fashion without relying on flooding-based techniques. We designed the underlying Neighbor Discovery and Controller algorithms required by Software Defined Wireless Sensor Networks. First we designed simple discovery algorithms to demonstrate the feasibility of our approach; next, we provided enhanced discovery algorithms that dealt with scalability issues. We executed experiments to check the occurrence of unidirectional links in a testbed, and implemented and tested our algorithms in a simulated/emulated environment. The discovery algorithms were tested on networks containing from 16 to 100 nodes, deployed randomly or as a grid. Each topology was tested in four link configurations: bidirectional links only, randomly selected unidirectional links, randomly selected nodes with increased range, and unidirectional links from the network controller to all other nodes. As a baseline, we considered a standard discovery algorithm used in Software Defined Wireless Sensor Networks, the Collect-based discovery. Employing unidirectional links yielded at least $90 \%$ packet delivered, whereas the Collectbased discovery yielded less $50 \%$ delivery on most cases, considering our experiments on topologies with unidirectional links. The final contribution is an asynchronous Radio Duty Cycling algorithm, to the best of our knowledge, the first to work under unidirectional links. When Radio Duty Cycling is present, the analysis showed that using unidirectional links is beneficial mostly in scenarios with long-reaching links.

Keywords: Communication protocols. Wireless. Internet of things. Distributed systems. 


\section{List of Figures}

Figure 1 - Graph example. . . . . . . . . . . . . . . . . . . . 19

Figure 2 - Communication patterns. . . . . . . . . . . . . . 22

Figure 3 - Types of radio duty cycling. . . . . . . . . . . . . . . . . . 23

Figure 4 - Software Defined Networking Architecture. . . . . . . . . . . . . . . 25

Figure 5 - IT-SDN node behavior. . . . . . . . . . . . . . . . . . . . 29

Figure 6 - Asynchronous slots example. . . . . . . . . . . . . . . 34

Figure 7 - Example of Searchligh schedule . . . . . . . . . . . . . . . 35

Figure 8 - Corner case. Circles represent successful packet delivery, the letter $\mathrm{x}$ represents delivery failure. . . . . . . . . . . . . . . . . . . . 42

Figure 9 - Example of delivery probability over time. . . . . . . . . . . . . . 43

Figure 10 - Nodes positioning used in the experiments. . . . . . . . . . . . . . 46

Figure 11 - Link quality estimation example. . . . . . . . . . . . . . . . . . 61

Figure 12 - Examples of topologies with unidirectional links: . . . . . . . . . . 67

Figure 13 - Sample network to controller discovery. . . . . . . . . . . . . . . . . . 69

Figure 14 - Examples of preamble sampling techniques. ContikiMAC uses techniques to reduce energy footprint. . . . . . . . . . . . . . . . . 70

Figure 15 - Unidirectional RDC: the controller informs the sender when it should start preamble transmission. . . . . . . . . . . . . . . . . . 72

Figure 16 - Unidirectional RDC detailed timing diagram. . . . . . . . . . . . 72

Figure 17 - Example of packets missed due to large clock drift. . . . . . . . . . . . 73

Figure 18 - Pure CSMA. Metric: Data delivery. Link setting: Bidirectional links only. 81

Figure 19 - Pure CSMA. Metric: Data delivery. Link setting: Controller to all. . . . 82

Figure 20 - Pure CSMA. Metric: Data delivery. Link setting: Nodes with increased range. . . . . . . . . . . . . . . . . . . . . 82

Figure 21 - Pure CSMA. Metric: Data delivery. Link Setting: Random unidirectional links. . . . . . . . . . . . . . . . . . . . 83

Figure 22 - Pure CSMA. Metric: Data delay. Link setting: Bidirectional links only. 84

Figure 23 - Pure CSMA. Metric: Data delay. Link setting: Controller to all. . . . . 84

Figure 24 - Pure CSMA. Metric: Data delay. Link setting: Nodes with increased range. . . . . . . . . . . . . . . . . . . . 85

Figure 25 - Pure CSMA. Metric: Data delay. Link setting: Random unidirectional links. . . . . . . . . . . . . . . . . . 85

Figure 26 - Pure CSMA. Metric: Link discovery rate. Link setting: Bidirectional links only. . . . . . . . . . . . . . . . . . . . . . . 87

Figure 27 - Pure CSMA. Metric: Link discovery rate. Link setting: Controller to all. 88 
Figure 28 - Pure CSMA. Metric: Link discovery rate. Link setting: Nodes with increased range. . . . . . . . . . . . . . . . . . . . . . . 88

Figure 29 - Pure CSMA. Metric: Link discovery rate. Link setting: Random unidirectional links.

Figure 30 - Pure CSMA. Metric: Control overhead. Link setting: Bidirectional links only. . . . . . . . . . . . . . . . . . . . . . 9 90

Figure 31 - Pure CSMA. Metric: Control overhead. Link setting: Controller to all. . 90

Figure 32 - Pure CSMA. Metric: Control overhead. Link setting: Nodes with increased range. . . . . . . . . . . . . . . . . . . . . . . . 91

Figure 33 - Pure CSMA. Metric: Control overhead. Link setting: Random unidirectional links. . . . . . . . . . . . . . . . . . . . . . 991

Figure 34 - Duty cycled. Metric: Data delivery. Link setting: Bidirectional links only. 92

Figure 35 - Duty cycled. Metric: Data delivery. Link setting: Controller to all. . . . 93

Figure 36 - Duty cycled. Metric: Data delivery. Link setting: Nodes with increased range. . . . . . . . . . . . . . . . . . . . 93

Figure 37 - Duty cycled. Metric: Data delivery. Link setting: Random unidirectional links. . . . . . . . . . . . . . . . . . . . . 94

Figure 38 - Duty cycled. Metric: Link discovery rate. Link setting: Bidirectional links only. . . . . . . . . . . . . . . . . . . . . 995

Figure 39 - Duty cycled. Metric: Link discovery rate. Link setting: Controller to all. 96

Figure 40 - Duty cycled. Metric: Link discovery rate. Link setting: Nodes with increased range. . . . . . . . . . . . . . . . . . . . . 996

Figure 41 - Duty cycled. Metric: Link discovery rate. Link setting: Random unidirectional links. . . . . . . . . . . . . . . . . . . . . . . . 97

Figure 42 - Duty cycled. Metric: Data delay. Link setting: Bidirectional links only. 98

Figure 43 - Duty cycled. Metric: Data delay. Link setting: Controller to all. . . . . 98

Figure 44 - Duty cycled. Metric: Data delay. Link setting: Nodes with increased range. . . . . . . . . . . . . . . . . . . 99

Figure 45 - Duty cycled. Metric: Data delay. Link setting: Random unidirectional links. . . . . . . . . . . . . . . . . . . . . 99

Figure 46 - Duty cycled. Metric: Data delay after convergence. Link setting: Bidirectional links only. . . . . . . . . . . . . . . . . . . . 100

Figure 47 - Duty cycled. Metric: Data delay after convergence. Link setting: Controller to all. . . . . . . . . . . . . . . . . . . . . . . . . . . . 101

Figure 48 - Duty cycled. Metric: Data delay after convergence. Link setting: Nodes with increased range. . . . . . . . . . . . . . . . . . . . . . . 101

Figure 49 - Duty cycled. Metric: Data delay after convergence. Link setting: Random unidirectional links. . . . . . . . . . . . . . . . . . . . 102 
Figure 50 - Duty cycled. Metric: Control overhead. Link setting: Bidirectional links only. . . . . . . . . . . . . . . . . . . . . 103

Figure 51 - Duty cycled. Metric: Control overhead. Link setting: Controller to all. . 103

Figure 52 - Duty cycled. Metric: Control overhead. Link setting: Nodes with increased range. . . . . . . . . . . . . . . . . . . . . . 104

Figure 53 - Duty cycled. Metric: Control overhead. Link setting: Random unidirectional links. . . . . . . . . . . . . . . . . . . . . . . . . . . . . . 104

Figure 54 - Duty cycled. Metric: Energy usage. Link setting: Bidirectional links only.105

Figure 55 - Duty cycled. Metric: Energy usage. Link setting: Controller to all. . . . 106

Figure 56 - Duty cycled. Metric: Energy usage. Link setting: Nodes with increased range. . . . . . . . . . . . . . . . . . 106

Figure 57 - Duty cycled. Metric: Energy usage. Link setting: Random unidirectional links. . . . . . . . . . . . . . . . . . . 107 


\section{List of Tables}

Table 1 - Classification of Neighbor Discovery algorithms . . . . . . . . . . . 38

Table 2 - Parameters Values. . . . . . . . . . . . . . . . 44

Table 3 - Power and distance values. . . . . . . . . . . . . . . . 45

Table 4 - Asymmetry metric $(\mathrm{m})$ values - Power combination $1 \mathrm{x} 1 \ldots$. . . . . . 46

Table 5 - Packet delivery Node 1 - Power combination $1 \mathrm{x} 1 \ldots$. . . . . . . . 47

Table 6 - Packet delivery Node 2 - Power combination $1 \mathrm{x} 1 \ldots$. . . . . . . . 47

Table 7 - Asymmetry metric $(\mathrm{m})$ values - Power combination $1 \mathrm{x} 2 \ldots$. . . . . 47

Table 8 - Packet delivery Node 1 - Power combination $1 \times 2 \ldots$. . . . . . . 47

Table 9 - Packet delivery Node 2 - Power combination $1 \times 2 \ldots$. . . . . . . . 48

Table 10 - Asymmetry metric $(\mathrm{m})$ values - Power combination $3 \mathrm{x} 3 \ldots$. . . . . . . 48

Table 11 - Packet delivery Node 1 - Power combination $3 \times 3$. . . . . . . . . . . . . 48

Table 12 - Packet delivery Node 2 - Power combination $3 \times 3$. . . . . . . . . . . . . 49

Table 13 - Asymmetry metric (m) values - Power combination $3 \mathrm{x} 4$. . . . . . . . . 49

Table 14 - Packet delivery Node 1 - Power combination $3 \mathrm{x} 4$. . . . . . . . . . . . . 49

Table 15 - Packet delivery Node 2 - Power combination $3 \mathrm{x} 4$. . . . . . . . . . . . . 49

Table 16 - Asymmetry metric values - Heterogeneous setting: TelosB vs SensorTag (radio power $-15 d B m) \ldots \ldots . \ldots . \ldots 50$

Table 17 - Simple Controller Discovery algorithm example with unidirectional links only . . . . . . . . . . . . . . . . . . . . 56

Table 18 - Simple Controller Discovery algorithm example with bidirectional links . 56

Table 19 - Influence of history size $(\mathrm{N})$ on LQE metrics. . . . . . . . . . . . . . . 64

Table 20 - Reactivity study. . . . . . . . . . . . . . . . . 66

Table 21 - Loss threshold to remove a neighbor from the neighbor table. . . . . . . 66

Table 22 - Enhanced Controller Discovery algorithm example . . . . . . . . . . . 69

Table 23 - Combinations of algorithms tested. . . . . . . . . . . . . . . 77

Table 24 - Simulation parameters. . . . . . . . . . . . . . . . 78

Table 25 - IT-SDN parameters. . . . . . . . . . . . . . . . . 78

Table 26 - Memory usage. . . . . . . . . . . . . . . . . . . . 79

Table 27 - Energy parameters. . . . . . . . . . . . . . . . . . . . 80 


\section{List of abbreviations and acronyms}

\begin{tabular}{|c|c|}
\hline AODV & Ad hoc On-Demand Distance Vector \\
\hline API & Application Programming Interface \\
\hline BRA & Bidirectional Routing Abstraction \\
\hline CAL & Control Abstraction Layer \\
\hline $\mathrm{CD}$ & Controller Discovery \\
\hline $\mathrm{CTP}$ & Collection Tree Protocol \\
\hline DAL & Device Abstraction Layer \\
\hline DP & Delivery Probability \\
\hline ETX & Expected Transmission Count \\
\hline IoT & Internet of Things \\
\hline IETF & Internet Engineeting Task Force \\
\hline $\mathrm{IPv} 4$ & Internet Protocol version 4 \\
\hline IPv6 & Internet Protocol version 6 \\
\hline ISM & Industrial, Scientific and Medical (frequency band) \\
\hline IT-SDN & Improved TinySDN \\
\hline LLDP & Link Layer Discovery Protocol \\
\hline LLN & Low power and Lossy Networks \\
\hline LQE & Link Quality Estimation \\
\hline LQI & Link Quality Estimator \\
\hline MAC & Medium Access Layer \\
\hline MAL & Management Abstraction Layer \\
\hline ND & Neighbor Discovery \\
\hline NSAL & Network Services Abstraction Layer \\
\hline
\end{tabular}


OS

Operating System

PDR Packet Delivery Rate

PRR Packet Reception Rate

RDC Radio Duty Cycling

RFC Request For Comments

RPL Routing Protocol for Low power and lossy networks

SaaS Sensing as a Service

SDN $\quad$ Software Defined Networking

SDWSN Software Defined Wireless Sensor Networking

SNR Signal to Noise Ratio

TDMA Time Division Multiple Access

TSCH Time Slotted Channel Hopping

ULC Unidirectional Link Counter

USP Universidade de São Paulo

WSN Wireless Sensor Network 


\section{Contents}

$1 \quad$ INTRODUCTION $\ldots \ldots \ldots \ldots \ldots \ldots \ldots \ldots$

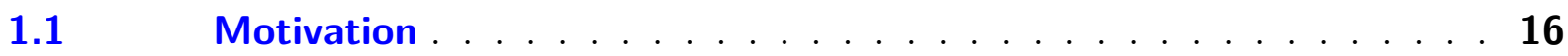

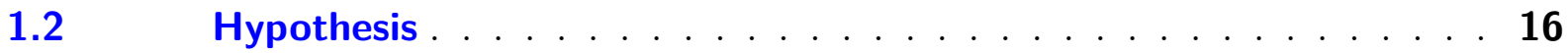

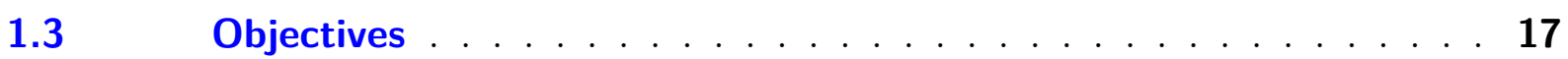

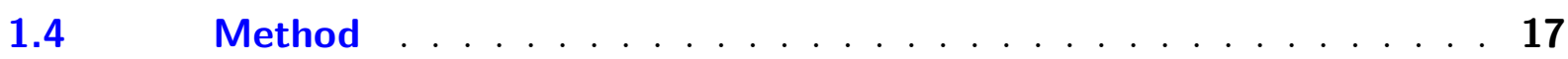

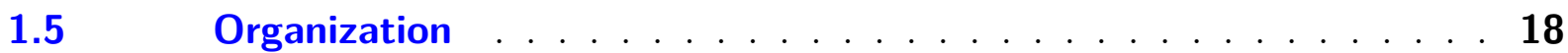

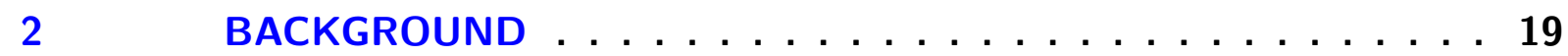

$2.1 \quad$ Graphs . . . . . . . . . . . . . . . . . . . 19

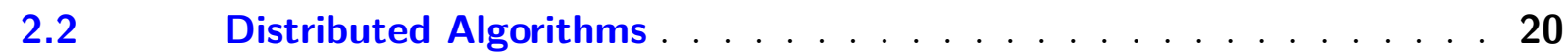

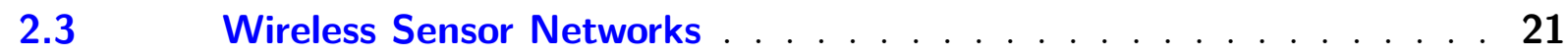

2.4 Software Defined Networking . . . . . . . . . . . . . . . . . 24

2.4.1 Software Defined Wireless Sensor Networks . . . . . . . . . . . . . . 26

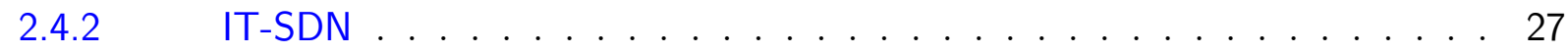

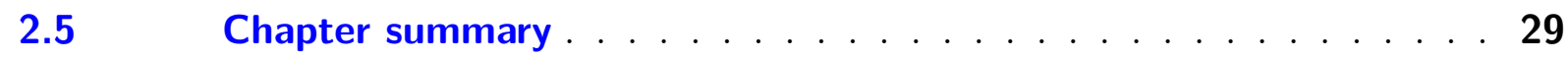

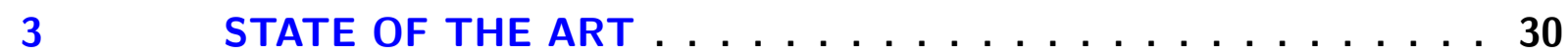

3.1 Studies on link asymmetry $\ldots \ldots \ldots \ldots$

$3.2 \quad$ Protocols adapted to asymmetric links . . . . . . . . . . 31

$3.3 \quad$ Neighbor Discovery $\ldots \ldots \ldots 33$

3.3.1 Generic Neighbor Discovery . . . . . . . . . . . . . . . . . 33

3.3.2 Neighbor Discovery in SDWSN . . . . . . . . . . . . . . . . . 37

$3.4 \quad$ Controller Discovery . . . . . . . . . . . . . . . . . 39

$3.5 \quad$ Chapter summary . . . . . . . . . . . . . . 40

$4 \quad$ LINK ASYMMETRY $\ldots \ldots \ldots \ldots \ldots \ldots \ldots \ldots$

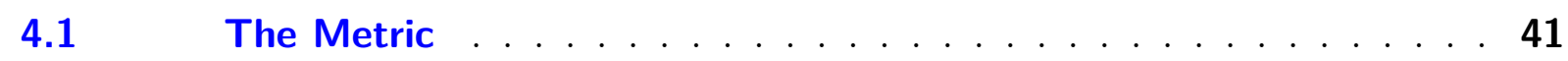

4.2 Method . . . . . . . . . . . . . . . . . 43

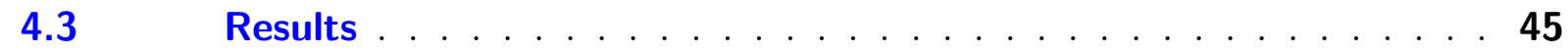

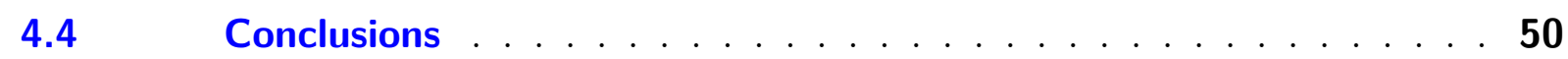

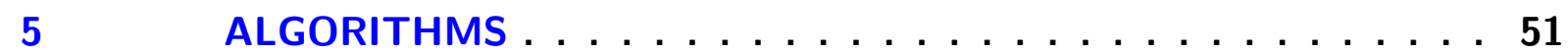

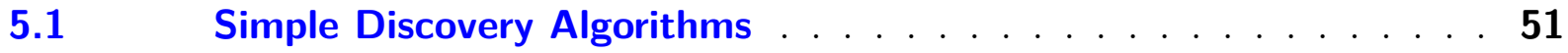

$5.1 .1 \quad$ Simple Neighbor Discovery Algorithm . . . . . . . . . . . . . . 51

5.1 .1 Implementation details . . . . . . . . . . . . . . . . 53

$5.1 .2 \quad$ Simple Controller Discovery Algorithm . . . . . . . . . . . . 53 
5.1.2.1 Implementation details . . . . . . . . . . . . . . . . 57

$5.2 \quad$ Enhanced Discovery Algorithms . . . . . . . . . . . . . 58

$5.2 .1 \quad$ Enhanced Neighbor Discovery . . . . . . . . . . . . . . 59

5.2.1.1 Neighbor discovery by overhearing . . . . . . . . . . . . . . . 59

5.2.1.2 Adaptive beacon interval . . . . . . . . . . . . . . . . 60

5.2.1.3 Link quality estimation $(\mathrm{LQE}) \ldots \ldots \ldots$. . . . . . . . . . . 60

5.2.1.4 Node Unreachability Detection . . . . . . . . . . . . . . . . . 61

5.2.1.5 Condensed Algorithmic description . . . . . . . . . . . . . . . . . 62

5.2.1.6 Implementation details . . . . . . . . . . . . . . . . . 62

5.2.2 Enhanced Controller Discovery . . . . . . . . . . . . . . . 67

5.2.2.1 Implementation details . . . . . . . . . . . . . . . . 69

$5.3 \quad$ Radio duty Cycling . . . . . . . . . . . . . . . . . . 69

5.3.1 Implementation details . . . . . . . . . . . . . . 74

$5.4 \quad$ Chapter summary . . . . . . . . . . . . . . 74

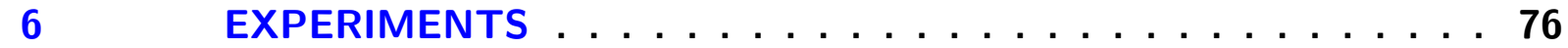

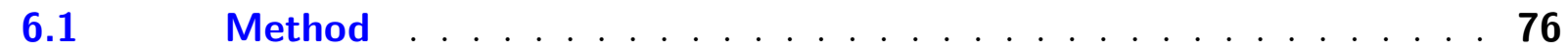

$6.1 .1 \quad$ Tools . . . . . . . . . . . . . . . . . 77

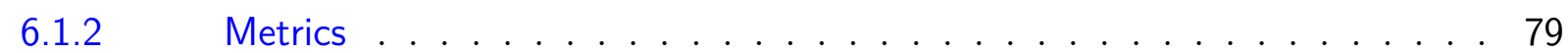

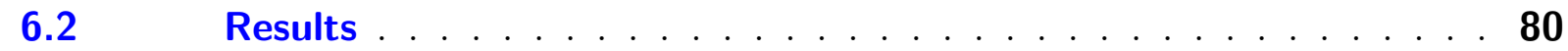

6.2.1 Simulation results without duty cycling . . . . . . . . . . 81

6.2.2 Simulation results with duty cycling . . . . . . . . . . . . 92

6.3 Conclusions . . . . . . . . . . . . . . . . . 107

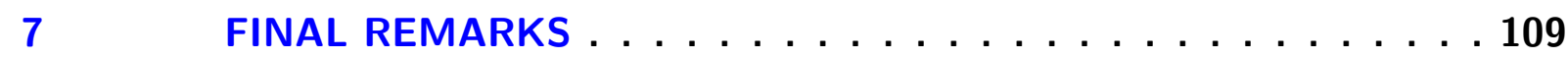

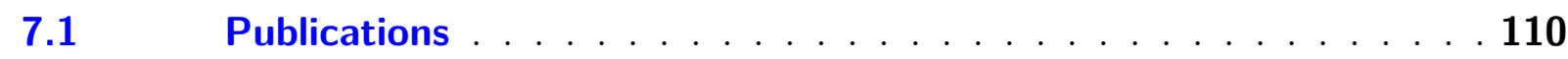

REFERENCES . . . . . . . . . . . . . . . . 112 


\section{Introduction}

The term Internet of Things (IoT) was coined in the late 90's, referring to the connection of everyday objects, sensors, and actuators to the internet. At first it was an abstract concept with science fiction undertones, now, it is a prolific research topic and a fast growing business, with billions of devices deployed around the world (AL-FUQAHA et al., 2015).

In parallel, Wireless Sensor Networks (WSN) started in the early 2000's as a thriving research area. Its main application is harvesting data from the environment, such as luminosity, humidity, temperature, and others. Although it was not focused on connecting devices to the internet, both WSN and IoT hold similar goals and requirements.

In 2003, the first version of the IEEE 802.15.4 standard was released (IEEE, 2011). This standard specifies both link and physical layers tailored for low power and low cost devices. It reduced the average power consumption from around one watt of IEEE 802.11 radios to tens of miliwatts. As a tradeoff, the IEEE 802.15.4 standard presents lower data rate and smaller maximum frame size, in comparison to IEEE 802.11 standard.

A key IoT requirement is a large enough addressing space to support the expected number of connected devices, in other words, the adequate version of the Internet Protocol is version $6(\operatorname{IPv} 6)$ instead of version $4(\mathrm{IPv} 4)$. To fulfill this requirement, the 6lowpan work group was formed in 2005 at the Internet Engineering Task Force (IETF), with the objective of providing a compatibility layer to compress long IPv6 headers into restricted IEEE 802.15.4 frames (KUSHALNAGAR; MONTENEGRO; SCHUMACHER, 2007). This research group's documents and specifications sealed the close bound between WSN and IoT.

While IoT and WSN applications are diverse (e.g., industry automation, eHealth, smart home, smart city), they all share common characteristics. The devices are batterypowered and cheap, incurring in limited processing capability and limited available memory.

The applications demand devices to be scattered over an area larger than the radio ranges, thus, a multihop communication strategy is required to send packets to a data sink. The research community developed a myriad of routing protocols with the unique WSN/IoT characteristics in mind. We classify the protocols in three waves.

The first wave of protocols aimed at balancing the tradeoff between reducing power consumption while increasing delivery rate (e.g. SPIN (HEINZELMAN; KULIK; BALAKRISHNAN, 1999)). Some protocols of this generation are even tied to the application, lacking generality, such as Directed Diffusion (INTANAGONWIWAT; GOVINDAN; 
ESTRIN, 2000). The second wave emerged with RPL (WINTER et al., 2012), the IPv6 Routing Protocol for Low-Power and Lossy Networks. It provided a standardized and generic protocol, which spanned a lot research towards its optimization.

The third wave of protocols focuses on implementing Software Defined Networking (SDN), a flexible routing paradigm that decouples the control plane from the data plane. On SDN, the control plane, responsible for the decision-making of packet forwarding routes, is logically centralized. This characteristic facilitates network management, enforcing network-wide policies, and calculating optimal routes.

\subsection{Motivation}

Presence of highly asymmetric or unidirectional links is an often neglected characteristic of low power wireless networks, which are often formed by IEEE 802.15.4 compliant devices. The formation of such links stem from many factors, for example, device positioning, signal reflection, non-isotropic antennas, and device heterogeneity, the latter being expected on Internet of Things deployments. We study some of these asymmetry-causing factors in Chapter 4, confirming the spontaneous formation of asymmetric links, whereas device diversity increases its occurrence and intensity.

Medium access and routing protocols are exposed to failures if oblivious to the possibility of unidirectional links existence. For example, any steps in the protocol execution requiring to send a message back to the sender are bound to fail. The extent of the disruption caused in the services provided ranges from performance degradation to complete failure, depending on the protocol specification.

Unidirectional links can be purposely formed by increasing the power output of selected network devices. Increasing transmission power holds the potential to reduce the average end-to-end path length, which saves energy at relay nodes and also reduces end-to-end packet delay. Devices with increased energy supply are good candidates to transmit at high power, extending the network lifetime.

In spite of the pitfalls and potential gains of unidirectional links, most protocols do not directly address this issue. At the same time, protocols that attempt to leverage unidirectional links are either non-efficient or case-specific, as we discuss in Chapter 3. The lack of thereof motivated us on pursuing an efficient and general solution for routing over unidirectional links.

\subsection{Hypothesis}

We believe that Software Defined Networking is an efficient and general-purpose solution for routing on Wireless Sensor Networks with unidirectional links. Our belief is 
based in the following observations:

1. The centralized control plane allows for easier route calculation than a distributed algorithm,

2. SDN has been successfully implemented in sensor networks, considering fully bidirectional networks, as we discuss in Chapter 3,

3. Intentional unidirectional links can be beneficial to the combination of SDN and WSN; since the controller has typically plenty of energy available, its transmission power can be increased, consequently, facilitating controller-to-node communication. Our experiments confirmed this conjecture, as increasing the controller transmission range reduced data delay in $17.9 \%$, in comparison to an equivalent fully bidirectional scenario.

\subsection{Objectives}

Our objective is to design an SDN-based WSN protocol that supports unidirectional links, and evaluate its performance. The main goal is dissected into the following secondary objectives:

1. Design a Controller Discovery algorithm that supports unidirectional links;

2. Design a Neighbor Discovery algorithm that supports unidirectional links;

3. Design a Radio Duty Cycling algorithm that supports unidirectional links;

4. Design experiments to demonstrate the algorithms effectiveness, while assessing the metrics of interest.

Neighbor discovery and controller discovery algorithms are underlying pieces of the SDN approach, Radio Duty Cycling algorithms are medium access techniques to reduce energy spent on communication. These topics are further discussed in Chapter 3.

\subsection{Method}

This section contains the method employed to accomplish the objectives.

First, we surveyed the discovery algorithms available in the literature. The goal was to check if any previous work deals with unidirectional links, and to identify commonly employed techniques. We discovered the existing algorithms rely on bidirectional links to work properly. 
Next, we adopted an iterative approach to develop our algorithms. We started by designing simple algorithms as a first likelihood indicator of our hypothesis confirmation. The results revealed SDN is a promising solution for routing in the presence of unidirectional links, once appropriate discovery protocols are provided.

However, the results also pointed scalability issues, the performance indicators degraded as the network size increased. Therefore, the simple algorithms were modified to decrease their control overhead. The same experiment scenarios were repeated, showing better scalability.

The last algorithm designed was the Radio Duty Cycling protocol; to the best of our knowledge, the first asynchronous RDC to support unidirectional links. The combination of discovery algorithms and RDC were also tested.

The experimental method is overviewed below, and thoroughly detailed in Chapter 6. All experiments included networks up to 100 nodes, varying network topology and types of link asymmetry. We assessed data delivery, data delay, control overhead, energy consumption, and link discovery rate metrics.

The experiments consisted of simulations executed on COOJA simulation tool. Each combination of factors was run 10 times with randomized simulation seeds, while average and confidence interval were calculated for statistical validity.

\subsection{Organization}

This document is organized as follows:

- Chapter 2, Background: basic concepts that are used throughout the thesis;

- Chapter 3, State of the art: a thorough analysis of relevant research papers;

- Chapter 4, Link asymmetry: an study on the causes of link asymmetry on Wireless Sensor Networks;

- Chapter 5, Algorithms: description of the algorithms developed and implementation details;

- Chapter 6, Experiments: experimental method and performance analysis;

- Chapter 7, Final remarks. 


\section{Background}

This chapter provides basic information and definitions that are used throughout the thesis. First, graphs and distributed algorithms are covered in Sections 2.1 and 2.2. Next, we present the main network-related concepts on Wireless Sensor Networks (Section 2.3), Software Defined Networks (Section 2.4), and, the intersection of those, Software Defined Wireless Sensor Networks (Section 2.4.1).

\subsection{Graphs}

In this thesis, graphs are a tool to represent computer networks: a vertex represents a network device, usually identified by its network address, edges represent a communication link between two network devices, and the edge weight represents link quality (for example, packet delivery rate). Since our study concerns undirectional links, we focus on directed graphs.

A graph is an abstract mathematical object composed of vertices and edges, which are connected to each other alternately. Vertices are graphically represented as circles, while edges are represented as lines connecting two vertices. Edges may be weighted, representing the cost of moving from one vertex to another. Figure 1 displays a graph example, containing 7 vertices and 8 edges.

Figure 1 - Graph example.
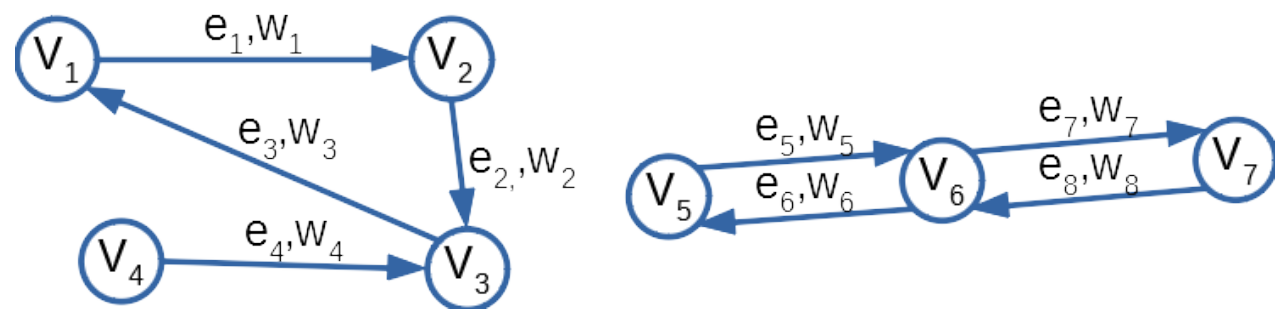

Source: author

More precisely, a graph is defined as a triple $G=(V, E, w)$, where $V$ is a set of vertices, $E \subseteq\left\{\left(v_{1}, v_{2}\right) \mid v_{1}, v_{2} \in V\right\}$ is a set of edges, an edge being an ordered pair of vertices, and $w: E \rightarrow \mathbb{R}_{+}^{*}$ is a weight function that assigns a positive number to an edge. Considering the example given in Figure $1, V=\left\{V_{1}, V_{2}, V_{3}, V_{4}, V_{5}, V_{6}, V_{7}\right\}, E=\left\{e_{1}:\left(V_{1}, V_{2}\right)\right.$, $\left.e_{2}:\left(V_{2}, V_{3}\right), e_{3}:\left(V_{3}, V_{1}\right), e_{4}:\left(V_{4}, V_{3}\right), e_{5}:\left(V_{5}, V_{6}\right), e_{6}:\left(V_{6}, V_{5}\right), e_{7}:\left(V_{6}, V_{7}\right), e_{8}:\left(V_{7}, V_{6}\right)\right\}$, and $w=\left\{\left(e_{1}, w_{1}\right),\left(e_{2}, w_{2}\right),\left(e_{3}, w_{4}\right),\left(e_{4}, w_{4}\right),\left(e_{5}, w_{5}\right),\left(e_{6}, w_{6}\right),\left(e_{7}, w_{7}\right),\left(e_{8}, w_{8}\right)\right\}$.

Next, let us define some graph concepts and graph properties used throughout the document: 
1. Path: a sequence of edges, in which the end point and starting point of consecutive edges must be the same. Example: $e_{4}, e_{3}, e_{1}$ in Figure 1;

2. Reverse path: given a path $p=\left\{\left(V_{1}, V_{2}\right),\left(V_{2}, V_{3}\right), \ldots\left(V_{n-1}, V_{n}\right),\right\}$, its reverse path $p_{r}=\left\{\left(V_{n}, V_{n-1}\right),\left(V_{n-1}, V_{n-2}\right), \ldots\left(V_{2}, V_{1}\right)\right\}$, if it exists, goes through the same vertices, but in the opposite direction. The path $\left(e_{8}, e_{6}\right)$ is the reverse path of $\left(e_{5}, e_{7}\right)$, considering Figure 1;

3. Reachability: a vertex $V_{A}$ is reachable from vertex $V_{B}$ if there is a path from $V_{B}$ to $V_{A}$. Example: $V_{1}$ is reachable from $V_{2}$ through the path $\left(e_{2}, e_{3}\right)$, in Figure 1 ;

4. Strongly connected: a directed graph is strongly connected if every vertex is reachable from every other vertex in the graph;

5. Strongly connected component: a strongly connected subgraph $G_{S}=\left(V_{S}, E_{S}\right)$ of a graph $G=(V, E)$. It is common practice to consider only the largest possible strongly connected subgraphs. Considering the graph in Figure 1, the set $S=\left\{\left\{V_{1}, V_{2}, V_{3}\right\},\left\{V_{4}\right\},\left\{V_{5}, V_{6}, V_{7}\right\}\right\}$ contains the vertex sets of all three strongly connected components;

6. Bidirectionally connected: a directed graph is bidirectionally connected if every vertex is reachable by a reversible path from every other vertex in the graph;

7. Bidirectionally connected component: a bidirectionally connected subgraph $G_{S}=\left(V_{S}, E_{S}\right)$ of a graph $G=(V, E)$. It is common practice to consider only the largest possible subgraphs. Considering the graph in Figure 1 , the vertices $\left\{V_{5}, V_{6}, V_{7}\right\}$ form the only non-unitary bidirectionally connected component.

\subsection{Distributed Algorithms}

A distributed system is a collection of nodes that collaborate on the execution of a computation by exchanging messages through a communication medium. A graph is used to represent the system structure, with nodes as vertex and communication links as edges (RAYNAL, 2013).

Each node is abstracted as a running process, able to receive messages, execute instructions, and send messages. All nodes in the distributed system run the same distributed algorithm, which is the description of these procedures (RAYNAL, 2013).

A set of assumptions are presupposed when designing a distributed algorithm. Any prior knowledge nodes may have must be explicitly stated, for example, if the identification number of its immediate neighbors are known or not. It is common practice to assume a node knows its own identification number. The communication channels should be specified according to its capacity, error rate, message ordering, and directionality (RAYNAL, 2013). 
Other properties and criteria to describe distributed algorithms are locality, synchronicity, termination, and complexity.

Locality. A distributed algorithm is local if every node in the distributed system only requires information from other nodes within a bounded hop distance. Otherwise, the algorithm is global, that is, a node may need information from any other node in the system (JHUMKA; MOTTOLA, 2016).

Synchronicity. Synchronous algorithms assume a synchronous distributed system, i.e, all nodes have access to a globally synchronized time base (clock). In addition, the algorithm execution occurs in rounds. At every round, there is a round of message transmissions, the next step only starts after all received messaged have been processed. On asynchronous algorithms, each node has its own separate clock, no synchronization is assumed, and the concept of execution round is not enforced (RAYNAL, 2013).

Termination. An algorithm terminates at the moment no more processing is required and there are not any messages scheduled for transmission. The nodes participating in the computation may or may not be aware it has ended, depending on the algorithm design. However, showing the algorithm terminates is an important step to demonstrate its correctness.

Complexity. The complexity of a distributed algorithm can be measured as the time cost or message cost. The first, time cost, is how long the algorithm takes to terminate. It can be assessed as the number of rounds for synchronous algorithms, while on asynchronous systems it can be assessed by the longest message transmission chain sequence. The latter, message cost, is total number of messages transmitted until the algorithm termination. On WSNs, the message cost closely relates to the amount of energy required to run the algorithm, since the radio represents most of the power consumption.

\subsection{Wireless Sensor Networks}

Wireless sensor networks (WSNs) are composed of nodes that use wireless radio technology to communicate, operating in a multihop fashion without any infrastructure, such as fixed access points. Nodes are typically resource constrained in terms of processing power, storage and memory, and rely on limited energy supply, e.g., batteries or energy harvesting techniques (MARGI, 2015).

As the purpose of a WSN deployment is usually harvesting information from the environment, the many-to-one communication pattern prevails. In this communication pattern, a set of nodes are designated as data sinks, responsible for collecting and storing the data gathered by other nodes, as exemplified in Figure 2a. Nonetheless, other communication patterns are also useful, albeit requested less often. For example, the one-to-many 
communication pattern is used for parameter configuration (Figure 2b); the many-to-many communication pattern can be used for data aggregation on application-specific settings (Figure 2c).

Figure 2 - Communication patterns.
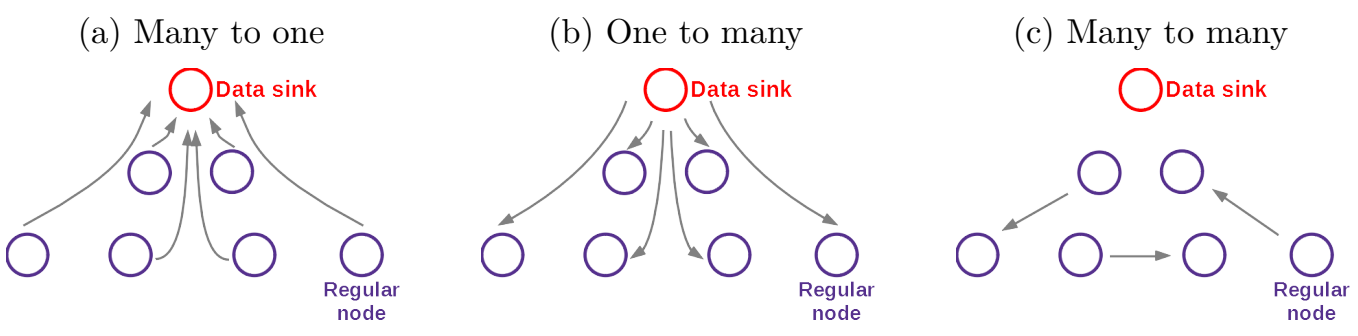

Source: author

The diversity of WSNs incurred in a lack of standardized upper layer protocols, with a multitude of proposed solutions, seeking to address unique characteristics of each deployment, application, or environment (AKKAYA; YOUNIS, 2005). This plurality reveals a noticeable difference to the layer 3 protocol landscape of the well known internet architecture. The narrow waist model defines the one-size-fits-all IP-based protocol suite, tailored for widespread connectivity. With that vision in mind, the IETF specified a set of IPv6 protocols tailored for constrained devices, such as the RPL routing protocol (WINTER et al., 2012) and a set of header compression rules (HUI; THUBERT, 2011).

The lower layers of the protocol stack present established standards, with IEEE 802.15.4 (IEEE, 2011) as the most widely used. The IEEE 802.15.4 defines physical layer parameters, and Medium Access Control (MAC) procedures (ALVES, 2014). Although it defines a few operation modes with varying energy-saving features, it is common to use IEEE 802.15.4 frame formats combined with its simplest mode of operation, while introducing a custom Radio Duty Cycling (RDC) layer on top of it.

There are three basic archetypes of RDC protocols: slot-based synchronous time multiplexing, asynchronous random access, and simultaneous transmissions. The overall goal is to minimize the number of transmitted packets and minimize the energy spent on idle listening, therefore, maximizing the time the radio is on low power state.

Synchronous time multiplexing uses dedicated time slots to transmit data, reducing collisions and delivery delay, at the risk of under-utilizing the available resources. Figure $3 \mathrm{a}$ exemplifies the transmission of a packet from node A to node B: upon a transmission request from upper protocol layers, the transmitter is required to wait until a previously scheduled transmission slot, dedicated to these two nodes, is available. An example is the Time Slotted Channel Hopping (TSCH) mode from IEEE 802.15.4e (IEEE, 2012).

Asynchronous random access performs best at low traffic load, since its working principle consists on periodic wake-ups and strobing packets. As shown in Figure 3b, a sender node A starts transmitting a wake-up signal as soon as the transmission request 
arrives. The wake-up signal eventually finds the wake-up window at node B, accomplishing the packet transmission. Since the medium is not dedicated to the communication between these two nodes, it is possible that packet collisions occur. B-MAC is a classic example of asynchronous RDC (POLASTRE; HILL; CULLER, 2004).

RDCs based on simultaneous transmission leverage constructive radio interference to ensure a packet transmission is received by all nodes in the network. The main drawback is only one node is able to disseminate information at a given time. For example, in Figure 3c, node $\mathrm{A}$ is required to wait until it is the sender of a simultaneous transmission round. Next, it transmits its packets to all its neighbors (nodes B and C), which, in turn, re-transmits the same packet immediately and simultaneously. The combined radio signals of both transmissions reach a common neighbor, node $\mathrm{D}$, which successfully receives the packet. Glossy marks the debut of simultaneous transmission protocols (FERRARI et al., 2011).

Figure 3 - Types of radio duty cycling.

(a) Slot-based

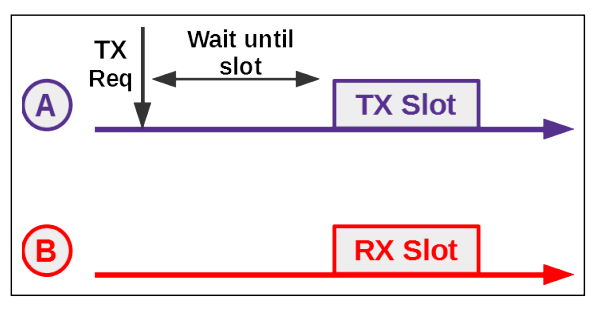

(b) Random access

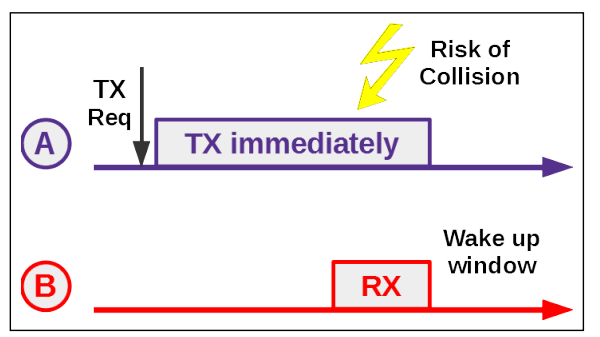

(c) Simultaneous transmission

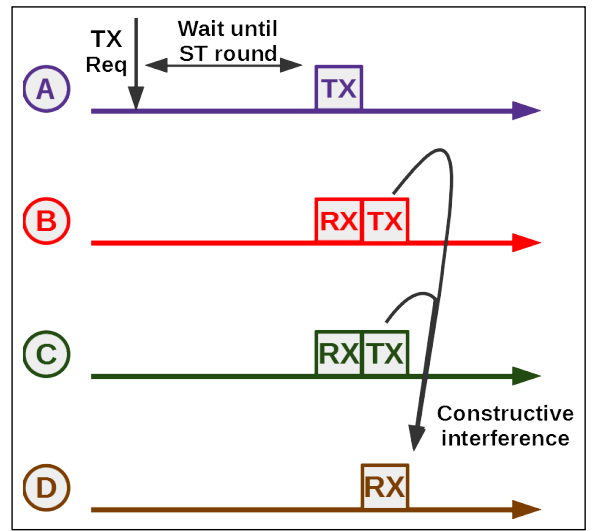

Source: author

Reducing energy consumption is important due to limited availability of this resource. For example, the TelosB platform operates on two AA batteries, the capacity of which is approximately $2000 \mathrm{~m} A h$ for alkaline type. A TelosB draws $\approx 20 \mathrm{~mA}$ while transmitting, $\approx 1.8 \mathrm{~mA}$ while processing, and $\approx 54.4 \mu \mathrm{A}$ on idle state. Therefore, reducing the radio duty cycle increases the network life time in many hours.

The CC2650 Sensortag platform, although it is a more modern and energy efficient platform, consuming only $\approx 6 \mathrm{~mA}$ on transmitting state, $\approx 50 \mu A$ while processing, and $\approx 1 \mu A$ on idle state, is even more energy constrained, as it operates on a CR2032 battery, whose typical capacity is $220 \mathrm{mAh}$.

WSN nodes are constrained not only in energy, but also on computational capacity. 
Using TelosB and CC2650 Sensortag as examples once more, their specifications are, respectively, as follows: $8 \mathrm{MHz}$ 16-bit microcontroller with $10 \mathrm{kB}$ of RAM and $48 \mathrm{kB}$ of ROM memory; $48 \mathrm{MHz} 32$-bit microcontroller with $20 \mathrm{kB}$ of RAM and $128 \mathrm{kB}$ of ROM memory.

Specialized operating systems (OSs) were crafted to address these limitations while providing a higher level abstraction to program these devices. TinyOS is an event driven OS developed by Berkeley University (LEVIS et al., 2005). It employs its own flavor of the $\mathrm{C}$ programming language, $\mathrm{Nes}$, with a large library of protocols ready to use. Its last official release was in 2012, without much development since then.

Contiki is another popular OS for constrained devices (DUNKELS; GRONVALL; VOIGT, 2004). It is implemented on C programming language, displaying its own protocol suit, called RIME stack, and implementations of IETF protocols. Its version 3.0 marked the end of its official development, in 2015. Two years later, in 2017, a fork project named Contiki NG was first released, focusing only on IETF protocols.

A more recent trend focuses on battery-less devices, operating exclusively from energy harvested from the environment. This class of networks requires specialized protocols, algorithms, and operating systems (YILDIRIM et al., 2018).

Wireless sensor networks have come a long way as a research area. This section provided a brief overview on the networks aspect, in terms of structure, protocols, and operating systems.

\subsection{Software Defined Networking}

Software Defined Networking (SDN) is a network routing paradigm with the objective of providing programmability and flexibility to multihop networks. The main characteristic of SDN is the separation between the control plane (routing decisions) and the data plane (actual packet switching). The control plane is logically centralized in the controller, an entity that determines the network behavior, while the network devices simply relay the packets based on the rules received from the controller. Unlike traditional routing, flow-based routing rules may consider any combination of packet fields, not only the destination address (KREUTZ et al., 2015).

The first action of an SDN-enabled device once it starts up is to establish communication with the network controller. This is trivial if the device is directly connected to the controller, otherwise an auxiliary controller discovery protocol is used. Next, the controller needs to build a local network representation. For that purpose, all nodes execute a neighbor discovery protocol to collect neighborhood information and send it to the controller. Once the controller has a representation of the network state, it sets the initial 
flow table rules. From this point on, the network is on a steady state, that is, SDN-enabled devices update the controller on topology changes, and the controller update flow rules according to the current policies.

Controller programmability enables easily changing routing criteria and enforcing policies, avoiding error-prone manual configuration. Additionally, the controller may provide an API so external applications can change operation parameters and current network behavior.

According to RFC 7426 - Software-Defined Networking (SDN): Layers and Architecture Terminology (HALEPLIDIS et al., 2015), a general model of the SDN architecture may be composed of several planes, as depicted in Figure 4. The Forwarding Plane or data plane concerns packet handling by the network devices, including forwarding, modification and dropping. The Control Plane is responsible for deciding the actions taken by the forwarding plane. The decision is informed to the forwarding plane by the means of a Southbound interface assuming a certain Device Abstraction Layer (DAL), i.e., an abstraction of the set of APIs and resources a network device contains.

Figure 4 - Software Defined Networking Architecture.

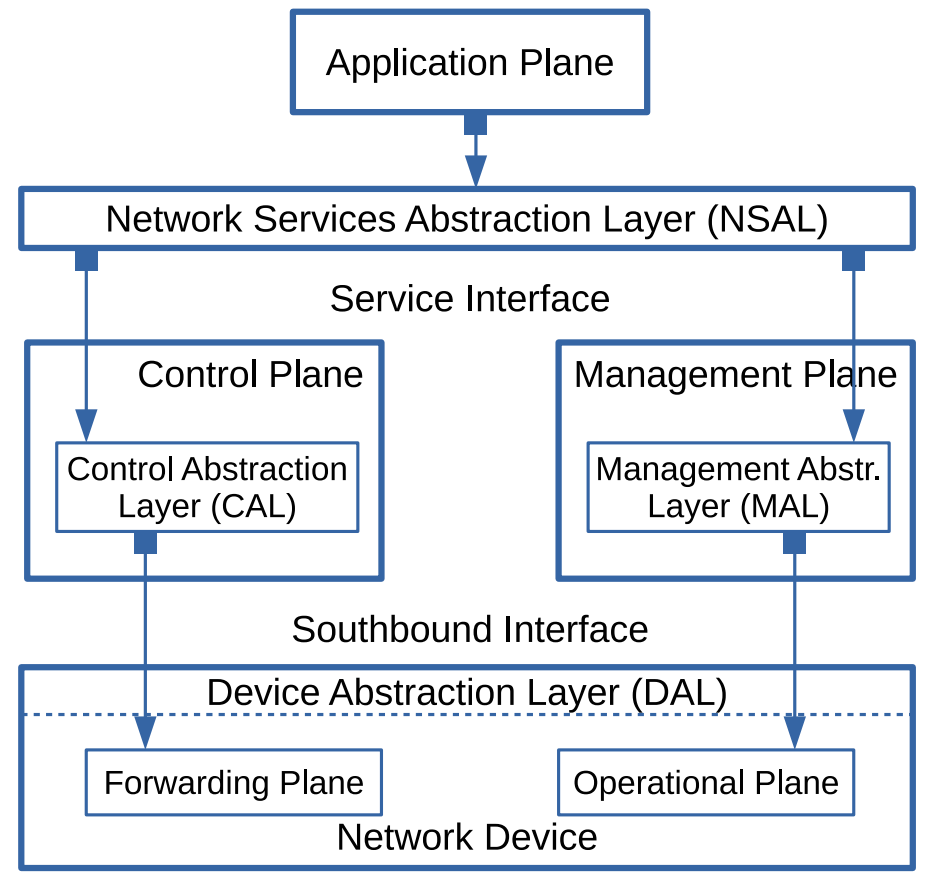

Source: adapted from Haleplidis et al. (2015, Figure 1)

The Operational Plane relates to information about the network device state, for example, the port status, queue lengths, remaining energy and temperature. The Management Plane is expected to be centralized, similar to the control plane. It takes decisions based on the operational plane status, and communicates with the device through the southbound interface.

The Application Plane contains the network applications that may use the control 
and management planes through the appropriate interfaces, namely the Network Services Abstraction Layer (NSAL), which may be used for communication between applications, the Control Abstraction Layer (CAL), which enables applications to manipulate the control plane and the Management Abstraction Layer (MAL), which provides access to the management plane information base. Network applications include path reservation, load balancing, and firewall.

Openflow is the first southbound protocol designed for wired networks (MCKEOWN et al., 2008). It first appeared in the context of the Clean Slate Program, as a way to rethink internet infrastructure and avoid its ossification. It is interesting to notice that although the term SDN was coined in the context of OpenFlow, the idea of network programmability have already been discussed before (CAMPBELL et al., 1999).

\subsubsection{Software Defined Wireless Sensor Networks}

The flexibility offered by SDN drew the attention of the WSN and IoT research community, as it could foster infrastructure reuse, node re-tasking, and increase the diversity of routing metrics. It is also a key technology for enabling the concept of Sensing as a Service (MARGI; ALVES; SEPULVEDA, 2017).

Early attempts to give SDN capabilities to Wireless Sensor Networks focused on porting OpenFlow to the constrained wireless scenario. Mahmud and Rahmani (2011) proposed FlowSensor, and used specific modeling tools to validate it. Simulation results show performance improvement in comparison to a traditional WSN routing algorithm, although the reference protocol was not specified. Sensor OpenFlow is another OpenFlowbased architecture for Software Defined Wireless Sensor Networks (LUO; TAN; QUEK, 2012). Sensor OpenFlow provides in-network data processing, and a mechanism to limit control traffic overhead. However, none of these works address the following issues of porting SDN to WSN: reduced maximum MAC layer frame size, node addressing scheme, and specification of discovery protocols.

The next Software Defined WSN approaches proposed their own southbound protocols. The main objective of SDN-Wise (GALLUCCIO et al., 2015) is to reduce the amount of information exchanged between the controller and the network nodes by introducing stateful flow tables. The authors present performance results from a simple 5-node testbed.

TinySDN is heavily based on the TinyOS operating system and uses the Collection

Tree Protocol as its basis (OLIVEIRA; MARGI; GABRIEL, 2014). It works based on a packet field called flow id, a flow-dependent identification. The results presented are limited to a 7-node linear topology.

IT-SDN (Improved TinySDN) is similar to TinySDN in the sense that it uses flow id 
to identify the network flows, however its architecture is OS independent (ALVES et al., 2017). IT-SDN is fully open source and implements source routed control packets, which reduces the requirement of flow table sizes. Performance analysis results were presented in a subsequent paper (ALVES et al., 2019), showing IT-SDN performs similar to the RPL protocol on the evaluated scenarios. IT-SDN allows changing the underlying discovery protocols required in SDWSNs, in contrast to the other works that present fixed protocols. IT-SDN is further discussed in Section 2.4.2, as it is the SDWSN framework employed to perform the experiments in this work.

Although there are several works testing SDWSN performance on static networks, mobile networks are seldom tested. Mobile nodes represent a potential pitfall to SDWSN functioning, since their existence increases the odds of a difference between the actual network topology and the network representation in the controller. This discrepancy is caused by the time it takes to detect the node has moved plus the time to inform the controller, once the detection occurs.

The controller can only take action after its network representation is updated. Hence, routes involving mobile nodes can become invalid before the controller can set a proper alternative route. Buratti et al. (2016) tested a dynamic scenario, in which some static nodes turn on and off throughout the experiment. Their experiments showed significant performance degradation.

As node mobility is not an issue on wired SDN, this topic is yet to be explored and is still an open issue in the SDWSN domain. Differently, the challenge of providing fault tolerance has been investigated in the context of wired SDN (REHMAN; AGUIAR; BARRACA, 2019), but is still a topic to be covered on SDWSNs. Fault tolerance is a relevant topic because the network controller is a single point of failure. Strategies that could be used to mitigate the impact of controller failure include: i) adding controller redundancy, and ii) falling back to a distributed routing protocol. Although some SDNWSN frameworks, e.g. TinySDN (OLIVEIRA; MARGI; GABRIEL, 2014), support multiple controllers, keeping the state of the controllers consistent is not discussed.

\subsubsection{IT-SDN}

IT-SDN (ALVES et al., 2019) is an SDN architecture specification for constrained devices. The control packets formats are designed to fit within the 127 bytes of IEEE 802.15.4 standard frames (IEEE, 2011). The underlying neighbor discovery and controller discovery protocols are not intertwined with the specification, enabling effortless replacement. The implementation showed a reduced memory footprint, fitting in TelosB motes (MEMSIC, 2011).

All sensor nodes are assumed to be SDN-enabled devices, with at least one of 
the nodes acting as a controller. The controller node should be provisioned with enough resources to support the processing and storage burdens bestowed upon it. A common practice it to employ a 32-bit computer, and attach a regular WSN mote to provide a compliant low power network interface. Packets are routed according to a flow identification number (flow id), defined by the application, while some of the control packets are routed according to the node address.

IT-SDN has its own Southbound protocol specification, composed of seven packet types, defined as follows:

1. Flow request: used by the nodes to query the controller about an unknown route.

2. Flow setup: the controller configures routes on the sensor nodes by sending flow setup packets. These packets may be transmitted in response to flow request packets or due to route recalculations performed by the controller.

3. Flowid register: informs the controller that the sender is a destination candidate for the specified flow id.

4. Acknowledgement: confirms the delivery of control packets. A packet is acknowledged based on the sequence number.

5. Neighbor report: contains node neighborhood information, which is sent to the controller at the neighbor discovery protocol request.

6. Data packet: encapsulates application layer packets.

7. Neighbor discover / controller discovery: used by the underlying discovery protocols.

Figure 5 summarizes the node behavior. A node forwards non-source-routed packets by checking the flow table and executing the actions it contains. If there is not a matching rule to the incoming packet, the node sends a flow request to the controller. The flow table stores routing information received from the controller, composed of the following columns: matching criteria (address or flow id), action (forward, drop, or receive), action parameter (typically the next hop), the number of times the entry has been used, and the age of entry.

Receiving a flow setup packet updates the flow table content, while data packets are forwarded to the upper layer. Acknowledgement packets are used to provide reliable delivery of control packets, which is achieved by periodic retransmissions. Therefore, receiving an ACK causes the corresponding timer to be disarmed.

In case of a flow table miss, the default action is to store the packet and to send a flow request. On the one hand, storing the packet increases the delay to deliver the first packet, particularly if the path to the controller has not been discovered yet. On the other 
Figure 5 - IT-SDN node behavior.

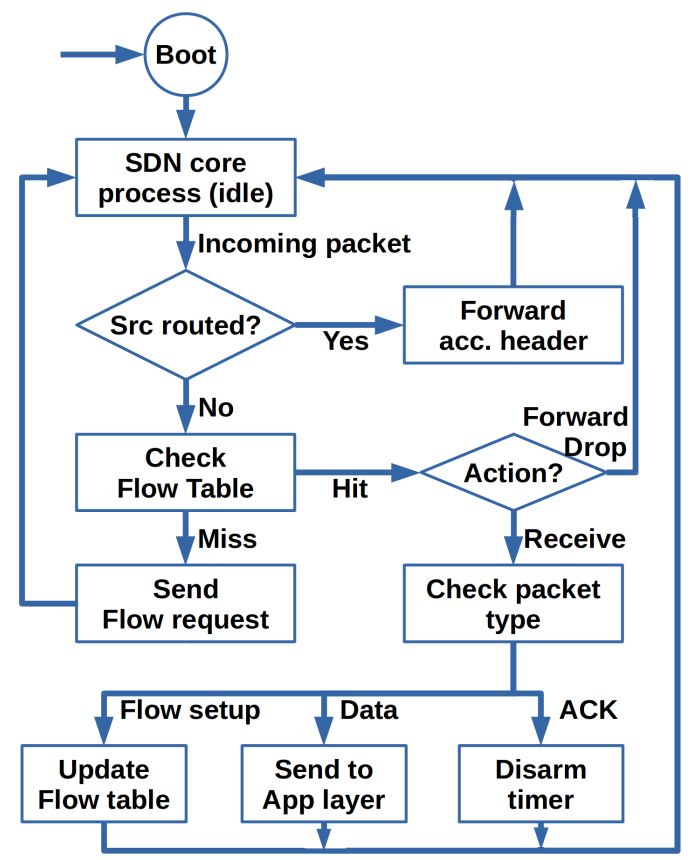

Source: Alves et al. (2019)

hand, the packet may be discarded instead, at the cost of decreasing the overall packet delivery rate. Similarly, the automatic flow request can be disabled if the controller is expected to eventually set all flows.

Opposed to the regular packets, source routed packets bypass the flow table check procedure and are forwarded according to contents of the source route header. The controller is the only node able to send source routed packets.

While IT-SDN specification is platform agnostic, the only available implementation at the time of this writing is based on the Contiki Operating System (DUNKELS; GRONVALL; VOIGT, 2004), with a custom controller implementation for x86 machines ${ }^{1}$. It has been compiled and tested on z1, TelosB, and CC2650 Sensortag motes.

\subsection{Chapter summary}

This chapter covered several baseline topics that are relevant to the research presented herein. First, graphs were introduced, a modeling tool used to represent a network topology. A basic understanding of what is a distributed algorithm and its properties is provided, as Wireless Sensor Networks are distributed systems in essence. The particular characteristics of WSNs, common terminology, and protocols are also described. Finally, we present the concept of Software Defined Networking, its intersection with WSN, and the realization of SDWSN by the IT-SDN framework, all of which are core to this work.

\footnotetext{
$\overline{1}$ Available at <www.larc.usp.br/users/cbmargi/www/it-sdn/>
} 


\section{State of the Art}

This chapter discusses the state of the art regarding studies on link asymmetry (Section 3.1), protocols for asymmetric networks (Section 3.2), neighbor discovery (Section 3.3), and controller discovery (Section 3.4). Lastly, we summarize the chapter by highlighting the gaps found in the state of the art in Section 3.5. The papers discussed in this chapter were found by searching keywords on relevant publication venues and on Google Scholar website. Whenever judged adequate, papers referenced by the found papers were also investigated.

\subsection{Studies on link asymmetry}

Wireless link asymmetry is related to link quality assessment, so we turn to the survey by Baccour et al. (2012). According to the authors, most link quality assessment methods are either receiver-sided or sender-sided. Consequently, most Link Quality Estimation (LQE) methods do not take link asymmetry into account.

Two exceptions were found in the survey: the Expected Transmission Count (ETX), and the Fuzzy Link Quality Estimator (F-LQE). The ETX metric (COUTO et al., 2005) estimates the link quality as $\frac{1}{P D R_{A B} * P D R_{B A}}$, in which $P D R_{A B} \in[0,1]$ is the packet delivery rate from $\mathrm{A}$ to $\mathrm{B}$, resulting in an ETX value in the interval $[1, \infty)$. Therefore, the lower the ETX estimation, lower are the chances of link asymmetry, since, given an ETX estimation, the maximum PDR difference is $1-\frac{1}{E T X}$. However, the actual maximum PDR difference may be any value in the interval $\left[0,1-\frac{1}{E T X}\right]$, making it inappropriate as an asymmetry metric, especially for high ETX values.

F-LQE (BACCOUR et al., 2010) uses fuzzy logic to combine packet delivery, link asymmetry, link stability, and channel quality. They defined asymmetry as the difference between the averaged delivery ratios, $\left|P D R_{A B}-P D R_{B A}\right|$. This definition is inappropriate if PDR varies over time, as we show in Chapter 4.

Sang, Arora and Zhang (2010) proposed ETF (expected number of transmissions over forward links), a link quality assessment method that can be used on unidirectional links. According to their evaluation, ETF consistently yielded higher PDR than ETX when used as link metric. Although the objective of this metric is not to quantify link asymmetry, the authors performed several experiments to study the link asymmetry phenomenon. The three main characteristics of their method are: (i) 100 packet samples, collected at $1 \mathrm{~Hz}$, (ii) each node transmits a trail of packets, that is, packet transmissions are not synchronized nor intertwined, (iii) the frequency band tested is $433 \mathrm{MHz}$. Their main findings regarding 
asymmetric links is that they occur often in low power wireless networks, and long reaching links are more likely to be unidirectional.

Zhou et al. (2004) studied the effect of non-isotropic antennas with Mica2 motes (CC1000 radio, operating at 433Mhz). Although they did not create an asymmetry metric, they showed that antenna rotation, battery level, and random manufacturing-effects play a role on link asymmetry, represented by the dispersion of RSSI values. Similar to Sang, Arora and Zhang (2010), they used a small non-intertwined sample (100 packets).

Srinivasan et al. (2010) provided a thorough analysis on low power wireless characteristics. Section 8 of their work, in particular, studies link asymmetry. Although they experiment on the $2.4 \mathrm{GHz}$ band, the shortness of their experiments hinder the drawing of further conclusions (only 2-seconds long).

From this analysis, we can identify three gaps in the current state of the art: (i) there is no dedicated metric to quantify link asymmetry, (ii) there is no dedicated method to assess link asymmetry, and (iii) a lack of extensive link asymmetry experiments on the $2.4 \mathrm{GHz}$ band.

\subsection{Protocols adapted to asymmetric links}

Bidirectional connectivity is a commonplace assumption in wireless routing protocols. For instance, the Routing Protocol for Low Power and Lossy Networks (RPL) (WINTER et al., 2012), the IoT routing protocol standardized by IETF, operates only over bidirectional links and requires this propriety to be verified by an underlying mechanism. The Collection Tree Protocol (CTP) (GNAWALI et al., 2009) also relies on bidirectional links to build the convergecast tree. Unidirectional links are ruled out as the underlying link assessment mechanisms detect it or due to data delivery failures. The Ad hoc On-Demand Distance Vector (AODV) protocol (PERKINS; BELDING-ROYER; DAS, 2003) is somewhat more robust to the presence of unidirectional links, however the exiting mechanisms to cope with them are not optimized.

Nonetheless, instead of being a problem to be dealt with, the unidirectional links could be used to improve the network performance. MOLSR-ASYM (BAI; CHEN, 2007) is a link state algorithm that detects unidirectional links by periodic uncoordinated beaconing. The beacons contain enough information to distinguish between inbound and outbound neighbors. Once the neighbors are classified, this information is propagated to the other nodes in the network and used to calculate the forwarding routes. On the other hand, link state algorithms are known to be inefficient in the context of WSN due to high message overheads.

Chen et al. (2013) proposed probabilistic routing algorithms focused on convergecast 
traffic. The network is sliced into concentric stripes centered at the destination. A node always transmits a packet $\mathrm{H}$ times, a parameter based on the expected link delivery rate and layer number. The packet is then forwarded with probability $\Gamma$, another parameter calculated to maximize end-to-end delivery rate. With this behavior, a lower layer node may overhear the packet and opportunistically transmit it to the sink, even if the link is unidirectional. The main drawback of this work is the lack of comparisons to other protocols and the reduced scope to convergecast traffic only. In addition, there are no considerations about the MAC layer.

Kim et al. (2016) assumes a network with a single sink able to reach any node in the network in one hop (but not the other way around). The objective is to provide efficient downward data transmission, which is achieved by a collaborative acknowledgement scheme to avoid expensive multihop control packets. The results show an advantage of the proposed protocol in comparison to RPL. However, it is difficult to generalize their solution to networks in which any link could be unidirectional.

The aforementioned works focused on the routing layer. But the medium access layer is also an appealing layer to solve the problem of unidirectional links. The following papers are similar as they create a bidirectional abstraction at the MAC layer, removing the responsibility of dealing unidirectional links from the routing layer.

COASYM-MAC is a receiver-initiated MAC protocol, that is, senders must await for a beacon from the target receiver. Once a receiver sends a beacon, senders contend to gain medium access by using random backoff timers. If it takes too long for the sender to hear a beacon, or if the data frame is not acknowledged, the link is assumed to be temporarily unidirectional and a procedure to find a 2-hop detour is performed. However, the neighbor discovery procedure is not explained; thus, the protocol would not work with fully unidirectional links. Also, packet losses due to collision are dealt the same way as losses due to unidirectionality, which affects performance on lossy links. The authors implemented the protocol on the simulator $\mathrm{OMNeT}++$; the experiments showed a performance improvement over a sender-initiated protocol (HASAN et al., 2016).

Bidirectional Routing Abstraction (BRA) (RAMASUBRAMANIAN; MOSSE, 2008) provides a full support for unidirectional links by computing multihop reverse paths to the unidirectional links. It uses an algorithm based on the Distributed Bellman-Ford, which floods the network with distance vector information. To curb the control overhead, the maximum length of the reverse path is limited. The authors claim BRA is easy to integrate with any routing protocol, although they recommend that the routing protocol should be aware of the unidirectional links, as it could interfere with expected delay of response packets (for instance, acknowledgements). The simulation results, based on the IEEE 802.11 standard, show that the AODV protocol performs better with BRA than using the traditional blacklisting mechanism. 
Unidirectional Link Counter (ULC) (KARNAPKE; NOLTE, 2015) is actually a cross-layer protocol, as it mixes functions from layer 2 and 3. The protocol is similar to AODV in the sense that Route Request and Route Response messages are used to discover routes, consequently, it is a flood-based protocol. These messages are also used to perform link discovery. If a link is unidirectional, the forwarding node relegates the forwarding task to its neighbors, expecting that at least one of them is able to countour the unidirectionality and find a reverse path. Both OMNeT++ simulations and testbed results show good performance in comparison to AODV (KARNAPKE; NOLTE, 2015).

It is noteworthy that BRA and ULC are focused on MANETs and use floodingbased messages. In the context of WSN it is desired to avoid flooding in order to reduce the number control packets throughout the network.

\subsection{Neighbor Discovery}

Neighbor Discovery (ND) is the process nodes execute at boot to acquire a representation of their current neighboring nodes. The ND may also be responsible for maintaining such information up-to-date, since it may change over time due to node mobility, battery depletion, or fading links.

The design of the neighbor discovery algorithm is governed by a set of assumptions regarding network conditions, available features, and node knowledge. For example, a designer may assume the network nodes are synchronized, have a global clock, and operate on a time-slotted basis. Oppositely, the network could be asynchronous. It may be assumed that some information is available to the nodes, such as the total number of nodes, the number of neighbors, or localization information.

The main metric used to evaluate an ND algorithm is the discovery latency, i.e., the time to discover all neighbors. Moreover, the protocol is expected to be energy efficient, which conflicts with the goal of reducing discovery latency. Thus, the challenge of discovery algorithm research is to balance these two conflicting objectives: reducing both discovery time and energy consumption.

We divide the state of the art neighbor discovery algorithms in two categories: Section 3.3.1 presents generic neighbor discovery algorithms, while Section 3.3.2 discusses the discovery strategies used in Software Defined Wireless Sensor Networks implementations.

\subsubsection{Generic Neighbor Discovery}

We classify the ND algorithms presented in this section as either duty cycle defining or non duty cycle defining. Duty cycle defining algorithms assume they have control over the radio, that is, the ND is able to turn the radio on and off. Let us start with duty cycle defining algorithms. 
McGlynn and Borbash (2001) proposed the birthday protocol, a probabilistic algorithm aimed at static ad hoc networks. The authors assumed a network with synchronized time slots. At the beginning of a time slot, each node randomly picks a radio state between transmitting, receiving, or low power. The discovery occurs if only one node transmits and at least one other node listens. The optimal probabilities were found to be dependent on the number of nodes, therefore this value must be estimated or known beforehand. The main drawback of this approach is that the discovery delay is unbounded, in other words, the probability distribution of the discovery latency has a long tail. As pointed out in the paper, a few links were not discovered at all in the simulation study. In addition, as the network is considered static, the neighborhood maintenance is not addressed.

Vasudevan et al. (2009) derive theoretical results for a neighbor discovery algorithm that does not require synchronization or knowledge about the number of nodes. The analysis of average delay starts from a simple slotted Aloha-based protocol, and then relaxes the main assumptions. Although it is assumed that the ND is able to control the radio, the low power mode is not considered. As it is a probabilistic protocol, the long-tail latency problem is present, on the other hand, the authors derive mechanisms to detect discovery termination. Also, this study lacks testbed and simulation experiments to confirm the theoretical analysis.

Protocols that ensure an upper bound on discovery latency are called deterministic. However, deterministic ND algorithms tends to yield higher average discovery delay than the probabilistic approach.

A common technique used in these algorithms to rescind the node synchronization requirement is to use asynchronous slots. An active slot is composed of two short beacon transmission at the time slot edges, while the radio is left on listening during the remaining time. Therefore, the overlapping of active slots triggers a discovery, as exemplified in Figure 6.

Figure 6 - Asynchronous slots example.

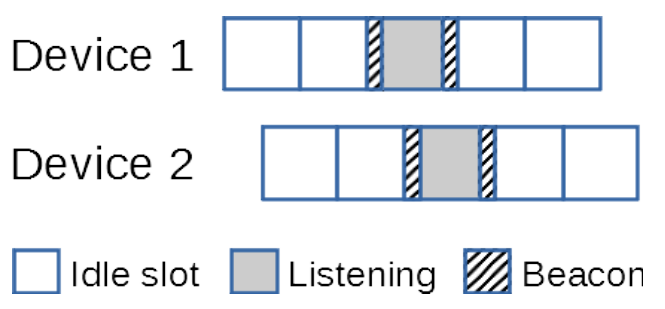

Source: author

The rationale of co-primality-based deterministic algorithms is to have each node $i$ pick a number $n_{i}$, with $n_{i}$ co-prime to the number $n_{j}$ picked by any neighboring node $n_{j}$. Every node $i$ transmits a discovery beacon at every $n_{i}$ time slots. Thus, due to the Chinese Remainder Theorem, every pair of neighbors will eventually discover each other. 
In the Disco protocol each node chooses two different prime numbers in order to ensure co-primality between every pair of nodes (DUTTA; CULLER, 2008). The authors discuss how to choose the prime numbers given a desired radio duty cycle. Simulation and testbed experiments show the efficiency of the proposal, as the discovery delay is comparable to the birthday protocol (MCGLYNN; BORBASH, 2001), depending on the prime number choice.

U-connect (KANDHALU; LAKSHMANAN; RAJKUMAR, 2010) requires each node to choose only one prime number $\mathrm{p}$. To ensure discovery in case nodes choose the same prime number, the node becomes active for $\frac{p+1}{2}$ slots in a row at every $p^{2}$ slots. The analytic results show that U-connect beats Disco in the average case. The authors also present testbed experiments for the U-connect algorithm. Purohit, Priyantha and Liu (2011) focused on the problem of neighbor discovery and group maintenance with mobile nodes. Their ND algorithm is similar to U-connect, while their maintenance mechanism requires node synchronization.

Searchlight (BAKHT; TROWER; KRAVETS, 2012) proposed an alternative to the use of prime numbers. Given a time slot schedule of length t slots, a node is active only in two slots: a fixed slot (anchor), and a random slot (probe). When two nodes are active at the same slot, they discover each other. It is assumed that anchor slots are unlikely to be overlap, therefore, the choice of the probe slot is randomized to increase chances of discovery. Anchor-probe slot overlapping is used to set an upper bound on discovery latency, while probe-probe slot overlapping is used to reduce average discovery latency. Simulation and testbed experiments show that, for a fixed duty cycle, searchlight overcomes U-connect in terms of discovery latency. Figure 7 contains an example of a Searchlight schedule, in which A denotes anchor slots transmitted at every $t=10$ slots, and $\mathrm{P}$ denotes probe slots transmitted at random between anchor slots. This schedule results in a $20 \%$ duty cycle and the discovery occurs in the hatched slots.

Figure 7 - Example of Searchligh schedule. A are periodic anchor slots and P are random probe slots.
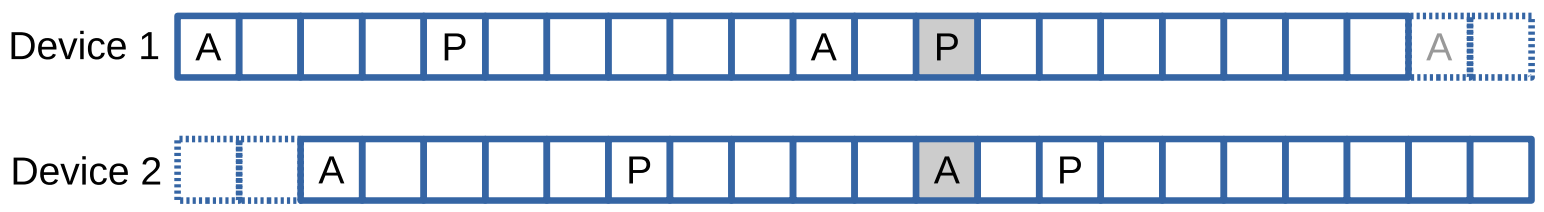

Source: author

Lightning protocol (WEI et al., 2016) improves upon Searchlight by using short probe slots. A similar strategy is used in Nihao, as it uses a "talk more listen less" approach, i.e., nodes listen in a small number of slots and send short beacons often (QIU et al., 2016). Chen et al. (2015) also improve upon Searchlight by proposing a general method to enhance time slotted neighbor discovery algorithms. 
There are other protocols that makes slight changes in the schedule design and are able to beat Searchlight (SUN et al., 2014; WANG; MAO; LIU, 2013).

Other works take advantage of mathematical theories and properties to design the schedule. Lai, Ravindran and Cho (2010) use a quorum system to ensure neighbor discovery in a time slotted network. They propose a grid and a cyclic quorum systems, and find the parameters to allow heterogeneous duty cycling. The use of codes has also been studied. Constant-weight codes and some variations are used by Keshavarzian et al. (2004), however, the radio sleep state is not considered. Meng, Wu and Chen (2014) devised an algorithm that builds an optimal code that defines the wake-up schedule in an asynchronous time slotted network. Their results show that this protocol also overcomes Searchlight.

Instead of discrete/slotted division of time, it is possible to consider transmission and listening at arbitrary time instants, as in the Griassdi protocol (KINDT et al., 2017). In fact it is possible to calculate the optimal beacon interval, scan interval and scan duration, considering a target duty cycle. As the transmissions are independent from the listening intervals, the authors devised a mechanism to reduce the two-way discovery delay. In addition, a maximum delay bound was calculated. BLEnd (JULIEN et al., 2017) is another discovery protocol, focused on Bluetooth Low Energy. The authors developed an optimizer to set the protocol parameters according to the user requirements. In spite of that, it works similarly to Griassdi (KINDT et al., 2017) and Nihao (QIU et al., 2016).

Nonetheless, it is not clear how duty cycle defining protocols interact with other protocols or applications. Since it is assumed that the neighbor discovery algorithm controls the radio state, the transmission windows are used to broadcast ND beacons only.

In Disco, the authors argue that the ND could provide the same interfaces as the MAC layer, but further discussion on how to integrate both MAC and ND functionalities is not included (DUTTA; CULLER, 2008). The authors of Searchlight relegates this matter to future work, while discussing whether the data transmission should leverage the underlying ND beacons, which would increase data delivery delay (BAKHT; TROWER; KRAVETS, 2012). Kandhalu, Lakshmanan and Rajkumar (2010) discuss that interoperability is possible regarding other nodes using low power listening MAC protocols, provided that the wake up intervals match. Also, as pointed out by Chen and Bian (2016), making the ND aware of the underlying MAC layer is a promising approach to improve energy efficiency.

The following protocols are non duty cycle defining as they do not assume the algorithm controls the radio. Cohen and Kapchits (2011) focus on the problem of continuous neighbor discovery, proposing to use different schemes for the initial discovery and for the information maintenance. Although it could be combined with more sophisticated algorithms, the authors test their proposal using a probabilistic scheme that requires an estimate of the number of neighbors. There is no comparison to other solutions in the 
simulation results.

The problem of neighborhood view consistency has been thoroughly studied by Jhumka and Mottola (2016). The authors developed a software device called pseudocrash detector and devised algorithms based on that theory. They proposed a global algorithm that works in networks with unidirectional links and a local algorithm for bidirectional networks. They implemented and tested only the latter algorithm and showed that routing algorithms benefit from their approach. As the proposed algorithm does not handle the duty cycling, ContikiMAC is used without modifications.

There is also a few branches of neighbor discovery algorithms concerning specific networks conditions or node characteristics such as directional antennas (JAKLLARI; LUO; KRISHNAMURTHY, 2007; CHEN; LI; VASILAKOS, 2017), multichannel links and multipacket reception (CHEN; BIAN, 2016), which are out of the scope of this research.

It can be observed from the surveyed papers that most of the ND proposals are duty cycle defining, while either assuming bidirectional links or do not discussing this matter. Table 1 summarizes the following characteristics of the protocols: duty cycle defining or non duty cycle defining, probabilistic or deterministic, implementation availability, working principle, and assumption about bidirectionality.

The focus of this work is on neighborhood initial discovery and neighboring information maintenance in asynchronous networks with unidirectional links. Integration with the other layers of the protocol stack is a primary concern. In addition, it should not be assumed the nodes have additional information about the network (such as globally synchronized clock, number of neighbors, or node localization).

\subsubsection{Neighbor Discovery in SDWSN}

Neighbor discovery is an essential task to Software Defined Wireless Sensor Networks. We discuss in this section the discovery algorithms adopted by the main SDWSN frameworks in the literature.

Flowsensor (MAHMUD; RAHMANI, 2011) and Sensor Openflow (LUO; TAN; QUEK, 2012), frameworks based on OpenFlow (MCKEOWN et al., 2008), are not explicit on how neighbor discovery occurs. Wired OpenFlow-based networks usually use Link Layer Discovery Protocol (LLDP) as their ND algorithm (ADAY; PASTOR; FERNÁNDEZ, 2015), consequently, our guess is that a variation of LLDP is used in the SDNWSN context.

TinySDN (OLIVEIRA; MARGI; GABRIEL, 2014) is built on top of the Collection Tree Protocol. Thus, it inherits its discovery capabilities, that is, it builds a bidirectional tree rooted at the controller, and uses ETX as link quality metric. 
Table 1 - Classification of Neighbor Discovery algorithms

\begin{tabular}{|c|c|c|c|c|c|}
\hline Work & $\begin{array}{c}\text { Duty cycle } \\
\text { defining }\end{array}$ & $\begin{array}{l}\text { Prob. } \\
\text { or Det. }\end{array}$ & $\begin{array}{c}\text { Implement. } \\
\text { Available }\end{array}$ & $\begin{array}{l}\text { Working } \\
\text { Principle }\end{array}$ & $\begin{array}{c}\text { Assumes } \\
\text { bidir. link }\end{array}$ \\
\hline $\begin{array}{l}\text { (MCGLYNN; BOR- } \\
\text { BASH, 2001) }\end{array}$ & Yes & $\mathrm{P}$ & No & $\begin{array}{l}\text { Random wake- } \\
\text { ups }\end{array}$ & No \\
\hline $\begin{array}{l}\text { (VASUDEVAN et al., } \\
2009 \text { ) }\end{array}$ & Yes & $\mathrm{P}$ & No & $\begin{array}{l}\text { Coupon collec- } \\
\text { tor problem }\end{array}$ & Yes \\
\hline $\begin{array}{l}\text { (DUTTA; CULLER, } \\
\text { 2008) }\end{array}$ & Yes & $\mathrm{D}$ & No & Co-primality & Yes \\
\hline $\begin{array}{l}\text { (KANDHALU; } \\
\text { LAKSHMANAN; } \\
\text { RAJKUMAR, 2010) }\end{array}$ & Yes & $\mathrm{D}$ & No & Co-primality & Yes \\
\hline $\begin{array}{l}\text { (PUROHIT; } \\
\text { PRIYANTHA; } \\
\text { LIU, 2011) }\end{array}$ & Yes & $\mathrm{D}$ & No & Co-primality & Yes \\
\hline $\begin{array}{l}\text { (BAKHT; } \\
\text { TROWER; } \\
\text { KRAVETS, 2012) }\end{array}$ & Yes & $\mathrm{D}$ & No & $\begin{array}{l}\text { Anchor slot } \\
\text { and probe slot }\end{array}$ & Yes \\
\hline (WEI et al., 2016) & Yes & $\mathrm{D}$ & No & $\begin{array}{l}\text { Anchor slot } \\
\text { and probe slot }\end{array}$ & Yes \\
\hline (QIU et al., 2016) & Yes & $\mathrm{D}$ & No & $\begin{array}{l}\text { "Talk more lis- } \\
\text { ten less" }\end{array}$ & No \\
\hline (SUN et al., 2014) & Yes & $\mathrm{D}$ & No & $\begin{array}{l}\text { Anchor slot } \\
\text { and probe slot }\end{array}$ & Yes \\
\hline $\begin{array}{l}\text { (WANG; MAO; LIU, } \\
\text { 2013) }\end{array}$ & Yes & $\mathrm{D}$ & No & $\begin{array}{l}\text { Anchor slot } \\
\text { and probe slot }\end{array}$ & Yes \\
\hline $\begin{array}{l}\text { (LAI; RAVINDRAN; } \\
\text { CHO, 2010) }\end{array}$ & Yes & $\mathrm{D}$ & No & Quorum & Yes \\
\hline $\begin{array}{l}\text { (KESHAVARZIAN } \\
\text { et al., 2004) }\end{array}$ & Yes & $\mathrm{D}$ & No & Codes & Yes \\
\hline $\begin{array}{ll}\text { (MENG; } & \text { WU; } \\
\text { CHEN, 2014) } & \end{array}$ & Yes & $\mathrm{D}$ & No & Codes & Yes \\
\hline (KINDT et al., 2017) & Yes & $\mathrm{P}$ & No & $\begin{array}{l}\text { Continuous } \\
\text { time }\end{array}$ & Yes \\
\hline $\begin{array}{l}\text { (JULIEN et al., } \\
2017)\end{array}$ & Yes & $\mathrm{D}$ & No & $\begin{array}{l}\text { "Talk more lis- } \\
\text { ten less" }\end{array}$ & Yes \\
\hline $\begin{array}{ll}\text { (COHEN; } & \text { KAP- } \\
\text { CHITS, 2011) } & \end{array}$ & No & $\mathrm{P}$ & No & $\begin{array}{l}\text { Random wake- } \\
\text { ups }\end{array}$ & Yes \\
\hline $\begin{array}{l}\text { (JHUMKA; } \text { MOT- } \\
\text { TOLA, 2016) }\end{array}$ & No & - & Yes & $\begin{array}{l}\text { Pseudo-crash } \\
\text { detector }\end{array}$ & $\begin{array}{l}\text { Yes (tested } \\
\text { version) }\end{array}$ \\
\hline
\end{tabular}

Source: author 
SDN-Wise (GALLUCCIO et al., 2015) implemented its own Topology Discovery protocol. Nonetheless, it behaves similarly to the Collection Tree Protocol, since it works based on periodic beacons sent by the sink. The main shortcomings of the SDN-Wise discovery protocol are assuming links are bidirectional, unnecessary transmission of control packets due to the lack of adequate criteria to send topological information to the controller, fixed Topology Discovery transmission interval, and use of a hardware-dependent link quality metric.

IT-SDN (ALVES et al., 2019) presents a more flexible design in the sense that the discovery protocol is not fixed. As a default option, IT-SDN uses Contiki's implementation of the Collection Tree Protocol as discovery protocol.

Lastly, Theodorou and Mamatas (2017) provide two discovery algorithms, although they use the term "topology control". Both proposed algorithms rely on the controller inquiring target nodes in the network, followed by nodes sending information back to the controller. However, the authors do not provide how the controller-to-node routes, node-to-controller routes, and link quality information are established in the first place.

From this analysis, it is observed that most SDWSN architectures have not paid special attention to discovery protocols. It is expected that a well thought ND design will improve overall SDWSN performance.

\subsection{Controller Discovery}

While neighbor discovery is a process needed in most WSN deployments, Controller Discover (CD) is exclusive to the software defined networking domain. At the time an SDN-enabled node boots, it does not know how to get in contact with the controller, as its flow table is empty. Therefore, the node needs to run a CD algorithm to find a path towards the controller and ask for further routing information.

Controller discovery is simpler to achieve in wired networks, since there is either a dedicated control channel, or a flooding-based mechanism is affordable. As mentioned in Section 3.3.2, the existing SDWSN frameworks use neighbor discovery protocols similar to the Collection Tree Protocol, which is also used for the purpose of controller discovery (OLIVEIRA; MARGI; GABRIEL, 2014; GALLUCCIO et al., 2015; ALVES et al., 2019). A tree rooted at the controller is built, so the nodes store the best candidate towards the controller among its neighbors.

An issue with the collect-based strategy is that links are presumed to be bidirectional, since the reception of a beacon packet is interpreted as if the receiver is able to send messages to the sender. The problem becomes more difficult to solve without this assumption. To the best of our knowledge, currently there is no controller discovery protocol that handles unidirectional links. 


\subsection{Chapter summary}

The objectives of this research lies in the intersection of Software Defined Networking, Discovery Protocols, and Routing in networks with unidirectional links, all three topics surveyed in this chapter.

While software defined networking is a promising technology for routing in wireless sensor networks, the underlying discovery protocols in the known implementations are not optimized. The best discovery protocols focus on bidirectional networks and are oblivious to the other layers of the protocol stack; therefore, they are not easily integrated into full-stack applications. Lastly, to the best of our knowledge, routing solutions for networks with unidirectional links are either flooding-based or tailored for a specific network setting.

To cover the gap of efficient routing on a network with unidirectional links, we use SDN with the aid of efficient discovery protocols, as discussed in Chapter 5. 


\section{Link asymmetry}

This chapter serves the purpose of examining factors that lead to the formation of asymmetric links in low power wireless networks. The findings presented herein were published at the International Workshop on Very Large Internet of Things (VLIoT 2020) (ALVES; MARGI, 2020).

Wireless communication depends on clear radio signals to decode incoming packets. Therefore, a wireless communication link may become asymmetric - or even unidirectional due to unbalanced conditions at the receiver of each communicating node.

Examples of factors that cause such disparities are: (i) meteorological conditions, (ii) non-isotropic antenna radiation patterns (ZHOU et al., 2004), (iii) differences in transmission power, and (iv) multipath fading.

The effect of these factors tends to be magnified in low power and lossy networks (LLN) in comparison to other kinds of wireless networks, because: (i) the devices are low cost, incurring in low quality transceivers, (ii) LLNs are multi-hop and device placement is often carelessly planned, and (iii) different device models may have non-conforming output powers and receiver sensitivities. For example, CC2420 radio power output ranges from -25 to $0 \mathrm{dBm}$ (TEXAS INSTRUMENTS, 2004), while CC2650 radio power output ranges from -9 to $5 \mathrm{dBm}$ (TEXAS INSTRUMENTS, 2015).

Whichever is the asymmetry source, it affects link quality metrics, such as the signal to noise ratio (SNR), link quality indication (LQI), and packet delivery rate (PDR). Nonetheless, assessing the magnitude of link asymmetry from these metrics is not a trivial task.

To achieve the goal of characterizing the occurrence of asymmetric links in LLNs, we devise a metric for quantifying link asymmetry in Section 4.1. In Section 4.2 we describe the experiments to explore the influence of four factors to link asymmetry: distance, radio power, node positioning, and hardware heterogeneity.

The experiment results in Section 4.3 show that link asymmetry occurs even in homogeneous networks. Heterogeneity factors further increase network asymmetry, potentially leading to highly asymmetric links.

\subsection{The Metric}

Let us assume the existence of a metric $m \in[0,1]$ able to capture the asymmetry of a link between nodes $\mathrm{A}$ and $\mathrm{B}$, with $m=0$ meaning perfectly bidirectional, and $m=1$ 
meaning completely unidirectional.

Intuitively, in a completely unidirectional link, node A delivers close to $100 \%$ to node $\mathrm{B}$, but node $\mathrm{B}$ cannot deliver anything to node A. Analogously, an example of a perfectly bidirectional link is a link which delivers $100 \%$ in both directions.

A simple way to calculate $\mathrm{m}$, bearing in mind the aforementioned criteria, would be $m=\left|P D R_{A B}-P D R_{B A}\right|$, where $P D R$ stands for packet delivery rate, the subscript indicates the assessment direction, e.g., $\mathrm{AB}$ is the delivery rate of packets transmitted from $\mathrm{A}$ to $\mathrm{B}$, and |.| is the absolute value function.

However, this definition is problematic if the underlying delivery probability changes over time. For example, take the artificially crafted corner case shown in Figure 8. According to the previous definition of $\mathrm{m}$, this link would not be asymmetric at all, since the result is $m=\left|P D R_{A B}-P D R_{B A}\right|=50 \%-50 \%=0$.

Figure 8 - Corner case. Circles represent successful packet delivery, the letter x represents delivery failure.

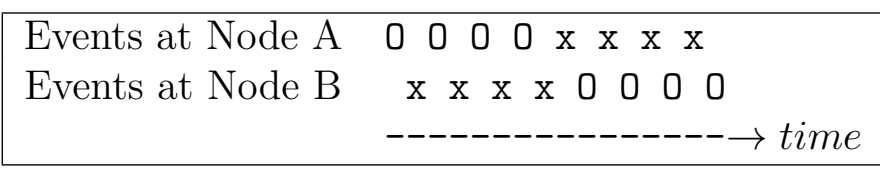

Source: author

However, a perfectly symmetric link does not match the intuitive expectations when observing the events in Figure 8, in this particular order. The time distribution of events should be taken into account to provide a more accurate assessment of the asymmetry.

Therefore, we define the link asymmetry metric, $m$, as the absolute value of instantaneous delivery probability difference, averaged during the observation time. Equation 4.1 comprises this definition, where $\mathrm{DP}(\mathrm{t})$ is the instantaneous Delivery Probability at time t, $t_{i}$ and $t_{f}$ are the initial and final observation times.

$$
m=\frac{\int_{t_{i}}^{t_{f}}\left|D P_{A B}(t)-D P_{B A}(t)\right| d t}{t_{f}-t_{i}}
$$

Estimating the unknown delivery probability $\mathrm{DP}(\mathrm{t})$ is a non-trivial task. As a simplistic example, consider the event trace presented in Figure 8. Suppose each node transmits packets at every time unit, Node A starts transmission at time $t=0$, while Node B starts at $t=0.5$. Furthermore, let us define $D P(t)=1$ if closest event in time is successful delivery, and $D P(t)=0$ otherwise.

According to this definition, the delivery probability from Node A to Node B is $100 \%$ from $t=0$ until $t=3.5$, and $0 \%$ onward, as the latest packet delivery successfully occurs at $t=3$ (the point $\mathrm{t}=3.5$ lies in the midpoint between the transition from success to failure). Analogously, the delivery probability from Node B to Node A is $0 \%$ from $t=0$ 
until $t=4$, and $100 \%$ onward. Figure 9 represents the evolution of delivery probabilities over time.

This setting results in an asymmetric index $m \approx 0.93$, indicating that our metric captures the unidirectionality of the link, whereas merely calculating the delivery rate difference would indicate no asymmetry at all.

Figure 9 - Example of delivery probability over time.

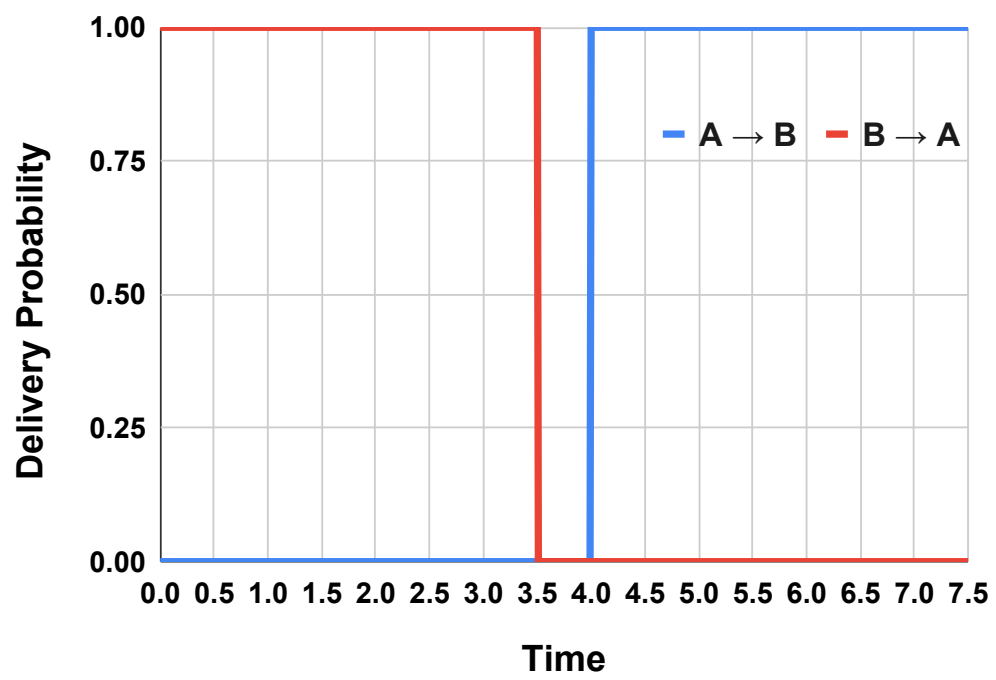

Source: author

The method to estimate the $\mathrm{DP}(\mathrm{t})$ can be arbitrarily complex, including multiple variables and statistics. The method we used, which compromises between complexity and accuracy, is described Section 4.2.

\subsection{Method}

We performed experiments with a pair of nodes transmitting packets to each other. We implemented the test software on Contiki OS 3.0, using the unreliable unicast primitive from the RIME stack (DUNKELS; ÖSTERLIND; HE, 2007), without any duty cycling mechanism. All experiments use IEEE 802.15.4 (IEEE, 2011) 2.4 GHz compliant radios.

Each node transmits unicast sequenced packets at fixed intervals $\Delta t$; the transmission schedules are shifted $\frac{\Delta t}{2}$ from each other. We empirically chose $\Delta t=100 \mathrm{~ms}$ in 1-hour long experiments. Each node logs the sequence number of received packets, enabling to detect which packets are lost, if any.

We used a sliding window technique to estimate delivery probabilities $D P(t)$. First, the packet reception data is converted to a vector named "delivery" of length 36000, filled with 0 s (packet not delivered) and 1s (success delivery). For example, the sequence $s$ of 5 events $s=$ (Success, Success, Failure, Failure,Success) would be mapped to a 
vector delivery of length 5 , with delivery $=[1,1,0,0,1]$. Since the experiment lasts for 1 hour, and a packet is sent at every 100ms, it yields 36000 data points.

For each data point, which corresponds to a packet transmission event, the delivery probability is calculated as shown in Equation 4.2, using a quadratic weight function weight $(x)=\frac{w_{m}-1}{w^{2}} x^{2}+1$, in which $w_{m}$ is the minimum weight, and the parameter $w$ sets the window's length $l$, calculated as $l=2 w+1$ [data points]. We used $w_{m}=0.1$ and $w=3$. The delivery probability is linearly interpolated on the time instants between packet transmissions.

$$
D P(t)=\frac{\sum_{i=t-w}^{t+w} \text { weight }(i-t) * \text { deliver }(i)}{\sum_{i=t-w}^{t+w} \text { weight }(i)}
$$

The resultant $D P(t)$ are fed into Equation 4.1 to calculate the asymmetry metric, m. Initially, we attempted using the method proposed by Jensen (2015). In this method, each data point is translated to a Gaussian function. However, feeding the resulting function in Equation 4.1, and using a numeric integrator to solve it, led to convergence issues. As an alternative, we used the sliding window provided above as an approximation.

We investigated the influence of four factors on link asymmetry. These factors were: (i) node relative distance, (ii) transmission power, (iii) nodes relative positioning, and (iv) node heterogeneity. Other factors are thought to influence link asymmetry, but these are generally difficult to control (e.g. multipath fading, external interference, relative humidity). We strive to keep these factors homogeneous by always running the experiments in the same climate-controlled location. The nodes are USB-powered to avoid voltage supply variations.

Table 2 - Parameters Values.

\begin{tabular}{cc}
\hline \hline Factor & Values \\
\hline Distance & close, moderate, far \\
\hline Power level & $1 \times 1,1 \times 2,3 \times 3,3 \times 4,7$ \\
\hline Positioning & Left-Right, Bottom-Top, Top-Bottom \\
\hline \multirow{2}{*}{ Hardware } & TelosB-TelosB, \\
& TelosB-SensorTag $(-15 d B m$, Left-Right $)$ \\
\hline \hline
\end{tabular}

Source: author

The range of factor values is displayed in Table 2. We tested several output power combinations. First, we used low transmisson power on TelosB motes, enabling small scale experiments. TelosB's radio transceiver, CC2420, has its output power configured by a 4 -bit register. Although it is possible to set any value between 0 and 31 , only the register values $3,7,11,15,19,23,27$, and 31 are documented with $\mathrm{dBm}$ power output on the datasheet (TEXAS INSTRUMENTS, 2004). We tested the following combinations 
of register values on TelosB-only experiments: $1 \mathrm{x} 1,1 \times 2,3 \times 3$, and $3 \times 4$, where a " $V_{1} \times V_{2}$ " pair represents the register value $V_{1}$ of the first node, and $V_{2}$ represents the register value of the second node. These values were chosen empirically to provide transmission power diversity within the constraints of physical space available to conduct the experiments.

We positioned the nodes apart at three distances: (i) close, in which PDR is expected to be nearly $100 \%$ in at least one of the directions; (ii) moderate, in which some packet losses are observed; and (iii) far, in which PDR is expected to be low. The actual distances between the pair of nodes depends on the radio power output, as shown in Table 3, and were empirically determined.

Table 3 - Power and distance values.

\begin{tabular}{cccc}
\hline \hline TelosB Power level & Close & Moderate & Far \\
\hline 1 & $0.20 m$ & $0.40 m$ & $0.50 m$ \\
\hline $3(-25 \mathrm{dBm})$ & $0.50 \mathrm{~m}$ & $0.70 \mathrm{~m}$ & $1.42 \mathrm{~m}$ \\
\hline $7(-15 \mathrm{dBm})$ & $\approx 6 \mathrm{~m}$ & $\approx 11 \mathrm{~m}$ & $\approx 14 \mathrm{~m}$ \\
\hline \hline
\end{tabular}

Source: author

The relative positioning of nodes is varied to check the influence of the antenna radiation pattern. We name the relative position as a pair of directions, each direction being the side facing the other mote, according to the convention shown in Figure 10a. From all the possible positioning combinations, we chose three: Top x Bottom (TxB), Bottom x Top (BxT), and Left x Right (LxR), as exemplified in Figures 10b, 10c, and 10d. The positionings Top x Bottom (TxB) and Bottom x Top (BxT) may seem equivalent at a first glance, but they were purposely chosen to check disparities in the antenna radiation pattern between the two devices.

The fourth factor is hardware. We test the connectivity between a pair of heterogeneous platforms, a TelosB mote (CC2420 radio) and a SensorTag mote (CC2650 radio). In this set of experiments, we only vary the distance, while setting the power output to $-15 \mathrm{dBm}$ (7, in CC2420 register configuration).

In total, 39 experiments were conducted, accounting for different combinations of distance, power levels, positioning, and hardware: 36 TelosB-only experiments (3 positions * 3 distances $* 4$ power level combinations $=36$ ) plus 3 heterogeneous experiments ( 1 position $* 3$ distances $* 1$ power level combination).

\subsection{Results}

The experiments' results are presented in tables, with three tables for each transmission power combination: one containing the asymmetry metric, other with the delivery rate in one direction, and a third table with the delivery rate in the other direction. 
Figure 10 - Nodes positioning used in the experiments.

(a) TelosB mote with named radio irradiation directions.

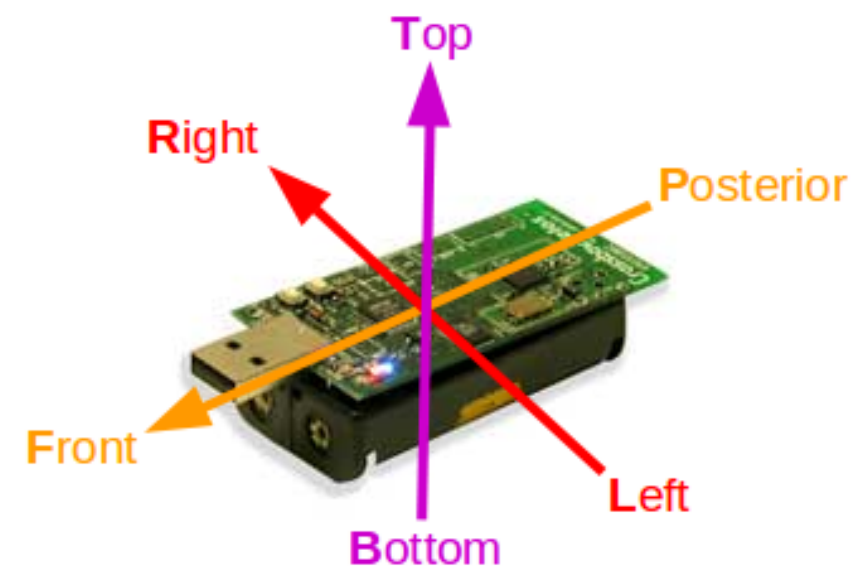

(b) Top x Bottom (TxB).

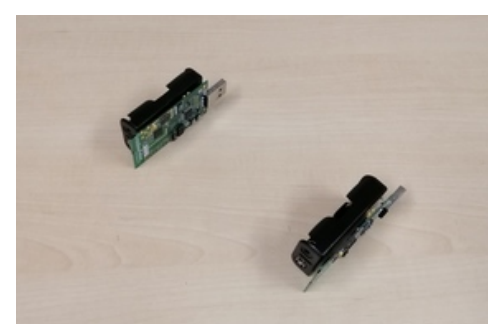

(c) Bottom x Top (BxT).

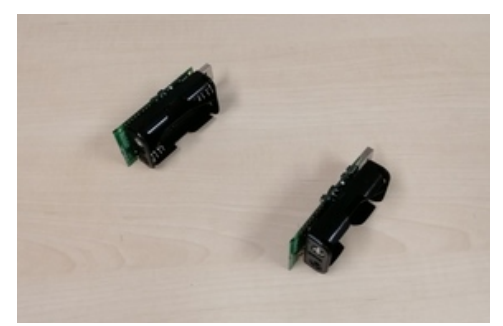

(d) Left x Right (LxR).

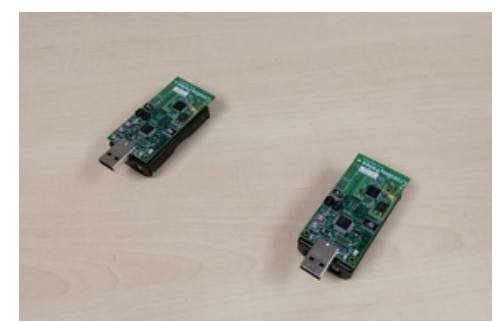

Source: author

Each row refers to the nodes' relative position: Top and Bottom (TxB), Bottom and Top (BxT), and Left and Right (LxR); the position naming convention is defined according to Figure 10a. Each column refers to the distance between the nodes: Close $(\mathrm{C})$, Moderate (M), and Far (F); the actual distances are defined according to Table 3.

Since the asymmetry metric and delivery rate is within the interval $[0,1]$, we use a color gradient to identify the results, with white cell background color representing 0 (no asymmetry / no delivery), and black cell background color representing 1 (fully unidirectional / all packets delivered).

Tables 4, 5, and 6 contains results for the power combination $1 x 1$. The only combination with high asymmetry is BxT at the far distance configuration. All the other combinations yielded near null delivery, with exception of LxR-close.

Table 4 - Asymmetry metric $(\mathrm{m})$ values - Power combination 1x1

\begin{tabular}{|c|c|c|c|c|}
\hline \multirow{3}{*}{ Positioning } & \multirow{2}{*}{$\begin{array}{l}\text { TxB } \\
\text { BxT }\end{array}$} & 0.00 & 0.00 & 0.00 \\
\hline & & 0.01 & 0.00 & 0.75 \\
\hline & LxR & 0.17 & 0.07 & 0.00 \\
\hline
\end{tabular}

Source: author

Distances 
Table 5 - Packet delivery Node 1 - Power combination 1x1

Source: author

\begin{tabular}{|c|c|c|c|c|}
\hline \multirow{3}{*}{ Positioning } & $\mathrm{TxB}$ & 0.00 & 0.00 & 0.00 \\
\hline & $\mathrm{BxT}$ & 0.01 & 0.00 & 0.76 \\
\hline & LxR & 0.86 & 0.07 & 0.00 \\
\hline
\end{tabular}

Distances

Table 6 - Packet delivery Node 2 - Power combination 1x1

Source: author

\begin{tabular}{cc|c|c|c|c}
\multirow{4}{*}{ Positioning } & TxB & 0.00 & 0.00 & 0.00 & $\mathbf{1}$ \\
& BxT & 0.00 & 0.00 & 0.01 & \\
& LxR & $\mathbf{0 . 8 5}$ & 0.00 & 0.00 & \multirow{2}{*}{0} \\
\cline { 2 - 5 } & \multicolumn{1}{c}{ C } & M & F
\end{tabular}

Distances

These results indicate three general findings: (i) Our asymmetry metric is more realistic than delivery rate difference, since LxR-close yielded nearly equal delivery in both directions ( $85 \%$ and $86 \%$ ), but non-negligible asymmetry (0.17); (ii) The high asymmetry at BxT-far suggests environmental effects, since shorter distances yielded no delivery in either direction; (iii) Node positioning is a determinant factor of link asymmetry and link quality, as the metric values varied sharply in a given column.

As expected, transmission power imbalance increases asymmetry, as observed in Tables 7, 8, and 9. Particularly in the LxR position, the node with higher transmission power was able to deliver more packets at every distance, while the other node delivered nearly no packets.

Table 7 - Asymmetry metric $(\mathrm{m})$ values - Power combination 1x2

\begin{tabular}{|c|c|c|c|c|}
\hline \multirow{3}{*}{ Positioning } & $\mathrm{TxB}$ & 0.13 & 0.00 & 0.00 \\
\hline & $\mathrm{BxT}$ & 0.33 & 0.00 & 0.00 \\
\hline & LxR & 0.91 & 0.50 & 0.66 \\
\hline
\end{tabular}

Source: author

Distances

Table 8 - Packet delivery Node 1 - Power combination 1x2

\begin{tabular}{|c|c|c|c|c|}
\hline \multirow{3}{*}{ Positioning } & $\mathrm{TxB}$ & 0.90 & 0.00 & 0.00 \\
\hline & $\mathrm{BxT}$ & 0.63 & 0.00 & 0.00 \\
\hline & $\mathrm{LxR}$ & 0.00 & 0.00 & 0.02 \\
\hline
\end{tabular}

Source: author

Distances

However, we observed three notable incongruities comparing the results of Power combination 1x2 to 1x1: (i) BxT-far became symmetric (no delivery in either direction); 
Table 9 - Packet delivery Node 2 - Power combination 1x2

Source: author

\begin{tabular}{cc|cc|c|c} 
& TxB & $\mathbf{0 . 9 0}$ & 0.00 & 0.00 & $\mathbf{1}$ \\
Positioning & BxT & $\mathbf{0 . 9 1}$ & 0.00 & 0.00 & \\
& LxR & $\mathbf{0 . 9 1}$ & $\mathbf{0 . 5 0}$ & $\mathbf{0 . 6 8}$ & 0 \\
& & C & M & F &
\end{tabular}

Distances

(i) LxR-close became highly asymmetric because the node with lower power failed to deliver packets; (iii) The node with lower power was able to deliver packets in the other close distance cases (TxB and BxT).

Since the experiments were executed in the same location, with the same motes powered by USB, effects such as multipath fading, manufacturing variations, and power supply oscillation are unlikely to be reason for the aforementioned incongruities. Rather, external interference is the presumable cause since the experiments were run at different times of the day. An spectrum analyzer could be used to confirm this hypothesis; we defer this task to future work.

As a consequence, link asymmetry can be seen as a transient quality in low power wireless networks. Furthermore, it is unreliable to use a 1-hour long data collection to infer steady state behavior.

Increasing the transmission power to $3(-25 \mathrm{dBm})$ in a symmetric setting reduced the overall link asymmetry to at most 0.1, as observed in Tables 10, 11, and 12 .

Delivery rates were all above $90 \%$ on close and moderate distances, but nearly $0 \%$ in the far distance configurations. This suggests that increasing the power decreases link asymmetry, since transmissions become less vulnerable to external interference.

Table 10 - Asymmetry metric (m) values - Power combination 3x3

\begin{tabular}{|c|c|c|c|c|}
\hline \multirow{3}{*}{ Positioning } & $\mathrm{TxB}$ & 0.10 & 0.05 & 0.01 \\
\hline & $\mathrm{BxT}$ & 0.07 & 0.05 & 0.00 \\
\hline & LxR & 0.10 & 0.03 & 0.00 \\
\hline
\end{tabular}

Source: author

Distances

Table 11 - Packet delivery Node 1 - Power combination 3x3

Source: author

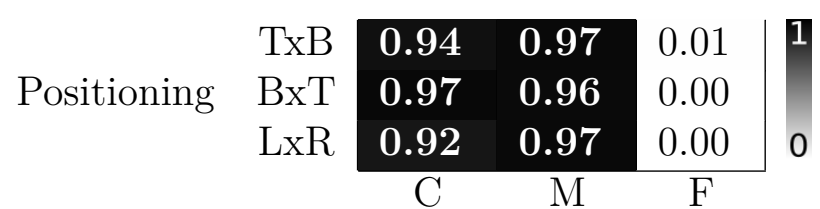

Distances 
Table 12 - Packet delivery Node 2 - Power combination 3x3

Source: author

\begin{tabular}{cccccc|c} 
& TxB & $\mathbf{0 . 9 1}$ & $\mathbf{0 . 9 7}$ & 0.00 & $\mathbf{1}$ \\
Positioning & BxT & $\mathbf{0 . 9 4}$ & $\mathbf{0 . 9 6}$ & 0.00 & \\
& LxR & $\mathbf{0 . 9 5}$ & $\mathbf{0 . 9 8}$ & 0.00 & \multirow{2}{*}{0} \\
& & C & M & F &
\end{tabular}

Distances

Increasing the transmission power of one node affects mostly the far region, as shown in Tables 13, 14, and 15. Close and moderate distances still presented high delivery rates in both directions, with the exception of LxR-moderate, which delivered no packets in either direction.

Table 13 - Asymmetry metric (m) values - Power combination 3x4

Source: author

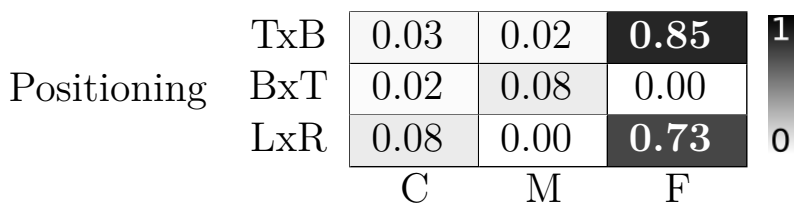

Distances

Table 14 - Packet delivery Node 1 - Power combination 3x4

\begin{tabular}{rrrrrr|r} 
& TxB & $\mathbf{0 . 9 6}$ & $\mathbf{0 . 9 7}$ & 0.14 & $\mathbf{1}$ \\
Positioning & BxT & $\mathbf{0 . 9 7}$ & $\mathbf{0 . 9 4}$ & 0.00 & \\
& LxR & $\mathbf{0 . 9 2}$ & 0.00 & 0.00 & \multirow{2}{*}{0} \\
& & C & M & F &
\end{tabular}

Source: author

Distances

Table 15 - Packet delivery Node 2 - Power combination 3x4

Source: author

\begin{tabular}{|c|c|c|c|c|}
\hline \multirow{3}{*}{ Positioning } & $\mathrm{TxB}$ & 0.98 & 0.99 & 0.99 \\
\hline & $\mathrm{BxT}$ & 0.98 & 0.96 & 0.00 \\
\hline & LxR & 0.97 & 0.00 & 0.73 \\
\hline
\end{tabular}

The increased power enabled packet delivery in one direction at the far distance, depending on the nodes positioning: TxB, BxT, and LxR yielded 99\%, 0\%, and 73\% delivery. The low power node delivered only a few packets, yielding high asymmetry only in TxB and LxR positions. This variation reinforces the influence of antenna radiation pattern on packet delivery and link asymmetry.

Lastly, the node heterogeneity study results are presented in Table 16. The asymmetry at close distance was low, in the same order of magnitude as found in the homogeneous 
nodes experiments. Low delivery rates in both directions also resulted in low asymmetry at the far distance.

Table 16 - Asymmetry metric values - Heterogeneous setting: TelosB vs SensorTag (radio power $-15 \mathrm{dBm}$ )

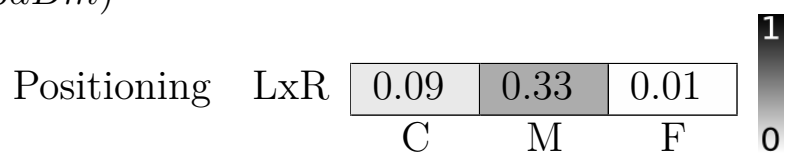

Source: author

Distances

However, at moderate distance, the asymmetry was 0.33 , almost 5 times larger than the asymmetry found on the homogeneous experiments with equal transmission power (Table 4 and Table 10). This result suggests that, in comparison to the TelosB mote, the SensorTag mote antenna either presents a different irradiation power, has a higher actual power output, or different receiver sensitivity.

\subsection{Conclusions}

Overall, the experiments show that link asymmetry is an undeniable phenomenon in low power wireless networks, with all studied factors playing a role on link asymmetry to some extent. In particular, we observed that long distance links are more prone to asymmetry than short distance links, telosB nodes transmissions are less powerful at the bottom side, and transmission power heterogeneity or hardware heterogeneity increase the chances of asymmetric links. However, predicting which specific combination of factors would result in a highly asymmetric link is not easily realized by a simple rule of thumb.

Even though the simplistic delivery rate difference would be an accurate asymmetry metric in some cases, our refined metric was able to grasp intricate asymmetry patterns in other cases. 


\section{Algorithms}

This chapter contains the core contributions of this work. First, we designed simple neighbor and controller discovery algorithms, described in Section 5.1. These algorithms demonstrate the feasibility of using SDWSN to route packets in a network with unidirectional links. Next, we crafted discovery algorithms aiming for efficiency, presented in Section 5.2. We provided a radio duty cycling algorithm that supports unidirectional links in Section 5.3. Lastly, we summarize the contents of this chapter in Section 5.4.

\subsection{Simple Discovery Algorithms}

This section describes a neighbor discovery algorithm and a controller discovery algorithm that are designed to operate on a network with unidirectional links, while keeping simplicity as a priority.

The neighbor discovery algorithm key idea is collecting inbound neighbors instead of outbound neighbors, which is sufficient information to allow the SDN controller to calculate routes (Section 5.1.1). The controller discovery algorithm was inspired by the Bellman-Ford algorithm, but the nodes only search paths towards one destination: the SDN controller (Section 5.1.2).

The content of this section was published at the XXXV Brazilian Communications and Signal Processing Symposium (SBrT) (ALVES; MARGI, 2017).

\subsubsection{Simple Neighbor Discovery Algorithm}

The function of neighbor discovery to a routing algorithm is searching for candidate relay nodes. On a classic network architecture running a distributed routing algorithm, the desired information is the set of outbound neighbors, since only an outbound neighbor is an eligible next hop towards a desired destination. However, finding an outbound unidirectional neighbor is not a trivial task, commonly achieved by flooding-based techniques.

On an SDN environment, the inbound neighborhood information is sufficient to build an accurate network representation at the centralized controller. The network topology is represented by a graph, whose basic elements are vertices and edges. The graph vertices represent the network nodes, which are added to the network representation when a Neighbor report packet is received by the controller. The Neighbor report sender and all of its inbound neighbors are added to the controller representation, along with a directed edge representing the link from each of the inbound neighbors to the Neighbor report sender. A bidirectional link between two nodes is represented at the controller by 
two directed edges. Therefore, gathering only inbound neighborhood is enough to build an accurate network representation at the controller, since every node and link is represented in the graph, assuming a functional neighbor discovery algorithm that detects all inbound neighbors. The controller can operate on the graph representing the network topology to calculate routes including unidirectional links using a local algorithm.

A simple way to achieve inbound neighbor discovery is by periodic asynchronous beaconing, as described in Algorithm 5.1. In other words, a beacon packet is transmitted at the timeout of a fixed interval timer. Whenever a node receives a beacon, it adds the sender to the neighbor table.

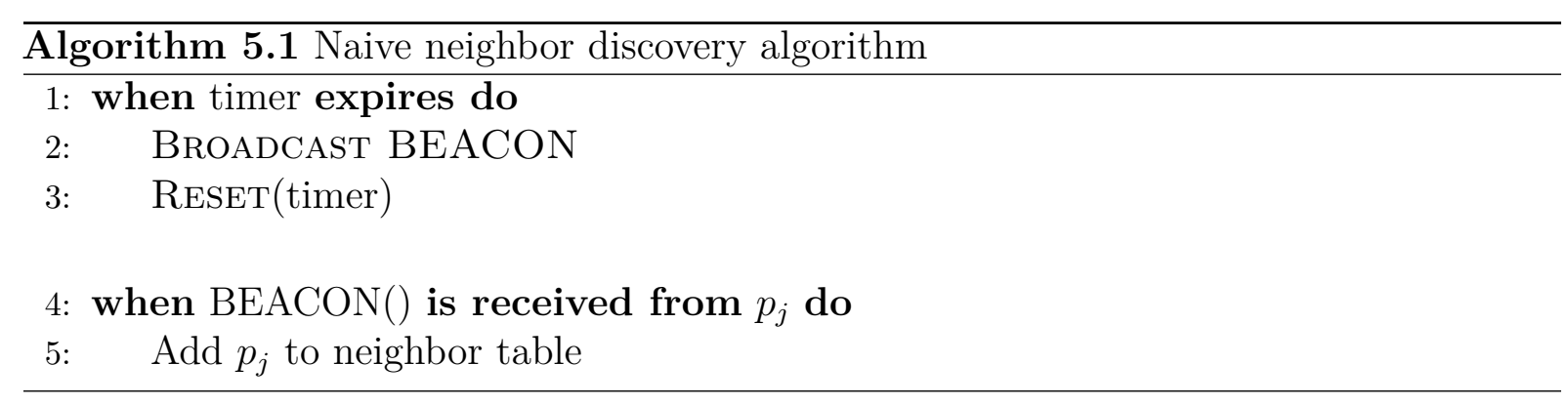

Two measures are taken to reduce the chances of beacon collision: (i) the time of the first beacon transmission is randomized; and (ii) each node picks a slight different timer interval (for example, based on the node id). The air time of a beacon transmission (milliseconds) is small compared to the transmission interval (minutes), therefore the likelihood of collision occurrence is low.

If the neighbor discovery beacon is received in the first attempt, the discovery delay, that is, the time it takes to discover a neighbor, equals the beacon interval plus the initial random delay. If a collision occurs, neighborhood detection would occur with high probability in the next attempt, as the beacon intervals are different. In this case, the discovery delay is twice the beacon interval plus the initial random delay. Therefore, the discovery delay is at the same order of magnitude as the beacon transmission interval.

However, there is a chance that two nodes start at the same time and choose the same beacon interval. In this case, their beacons would collide at every transmission attempt. The underlying MAC protocol would have to deal with this contention, but broadcast transmissions are usually unacknowledged and a failed transmission attempt is not detected or retransmitted. Therefore, in this specific case, the nodes would not be detected by their common outbound neighbors.

The message cost of the algorithm grows linearly with the network up-time, as there is not a mechanism to stop or modify the interval of the beacon transmissions. 


\subsubsection{Implementation details}

The simple neighbor discovery algorithm implementation was straightforward. It used Contiki's event timers to periodically transmit the ND beacons. The timers are adjusted with the reminder of the node address division by 10, as a way to randomize beacon transmissions. The beacon interval can be configured prior to network deployment, but it does not change during network execution. The beacons are transmitted throughout the entire node lifetime.

\subsubsection{Simple Controller Discovery Algorithm}

Controller discovery is the problem of deciding the next hop towards a given distinct node, for each node in a graph. If the graph is undirected, the typical strategy is to build a tree routed at the controller node, similar to the controller discovery protocols described in Section 3.4. On the other hand, this strategy does not work if the graph is directed, since receiving a message from a node does not imply the existence of the reverse communication link.

Bellman-Ford is a classic algorithm to calculate shortest paths between every pair of nodes in a graph. Algorithm 5.2 exhibits the pseudo-code of the distributed version of the algorithm. It requires the following conditions: the graph is undirected, edge weights are fixed, the number of nodes in the graph is known, each node knows its neighbors, and message transmissions are error free.

Node $i$ maintains the variable length ${ }_{i}$, a list of the current distances to the other nodes in the graph, initialized with zero at the i-th position and with infinity at all other positions. The nodes are numbered from 1 to $\mathrm{n}$, thus, the list index corresponds to the node identification. The other variable is routing_to ${ }_{i}$, whose $\mathrm{k}$-th position contains the next hop towards node k. Node $i$ also knows its set of neighbors, denoted by neighbor $s_{i}$ and the communication cost to each of its neighbors, denoted by $l g_{i}$.

At least one node in the graph needs to receive an external START message, which triggers a transmission of UPDATE messages to its neighbors, containing the current length list (lines 1-3). Upon receiving an UPDATE message, the node checks if the sender $\mathrm{j}$ is a better next hop towards any of the n nodes in the graph (lines 6-10). This is achieved

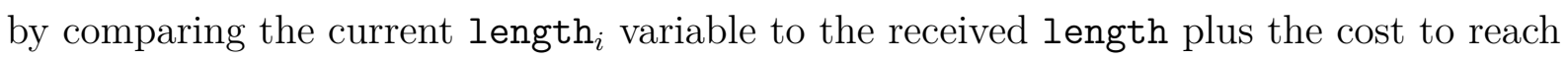
the sender. If the sender is a better next hop, the local length ${ }_{i}$ and routing_to variables are updated and a new UPDATE message is transmitted to all neighbors (lines 11-13).

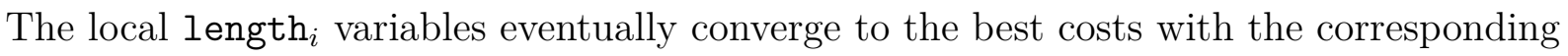
routing_to ${ }_{i}$ values (RAYNAL, 2013).

The message cost (i.e. the number of control messages transmitted) of the distributed Bellman-Ford algorithm is known to be exponential in the worst case, although the time 


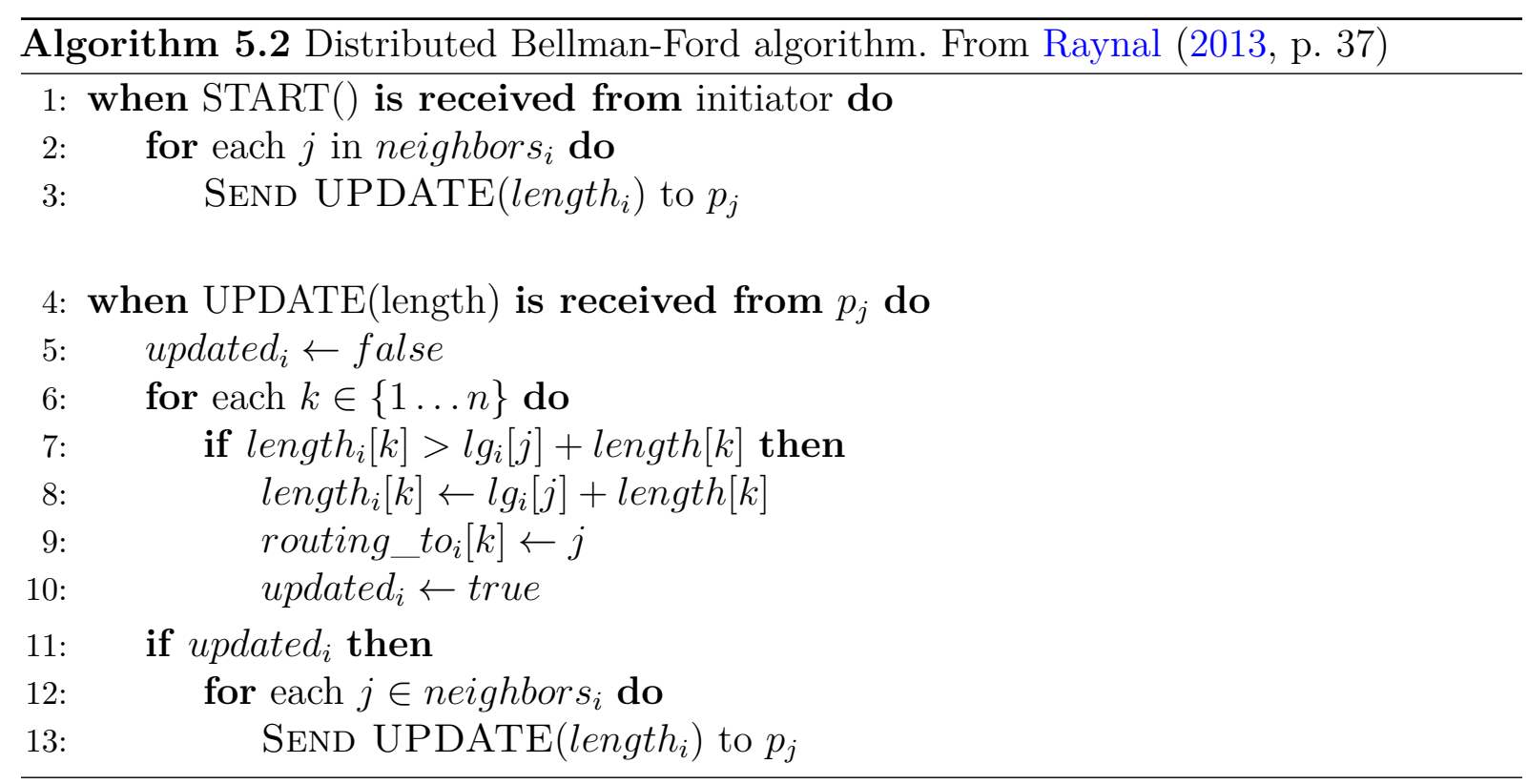

complexity is linear (AWERBUCH; BAR-NOY; GOPAL, 1994). However, the exponential number of messages scenario is unlikely in computer networks scenarios, as it would need a very peculiar order of messages and multiple edges between a pair of nodes. The UPDATE message length is constant and proportional to the number of nodes in the network, the same applies to the local memory cost of the algorithm.

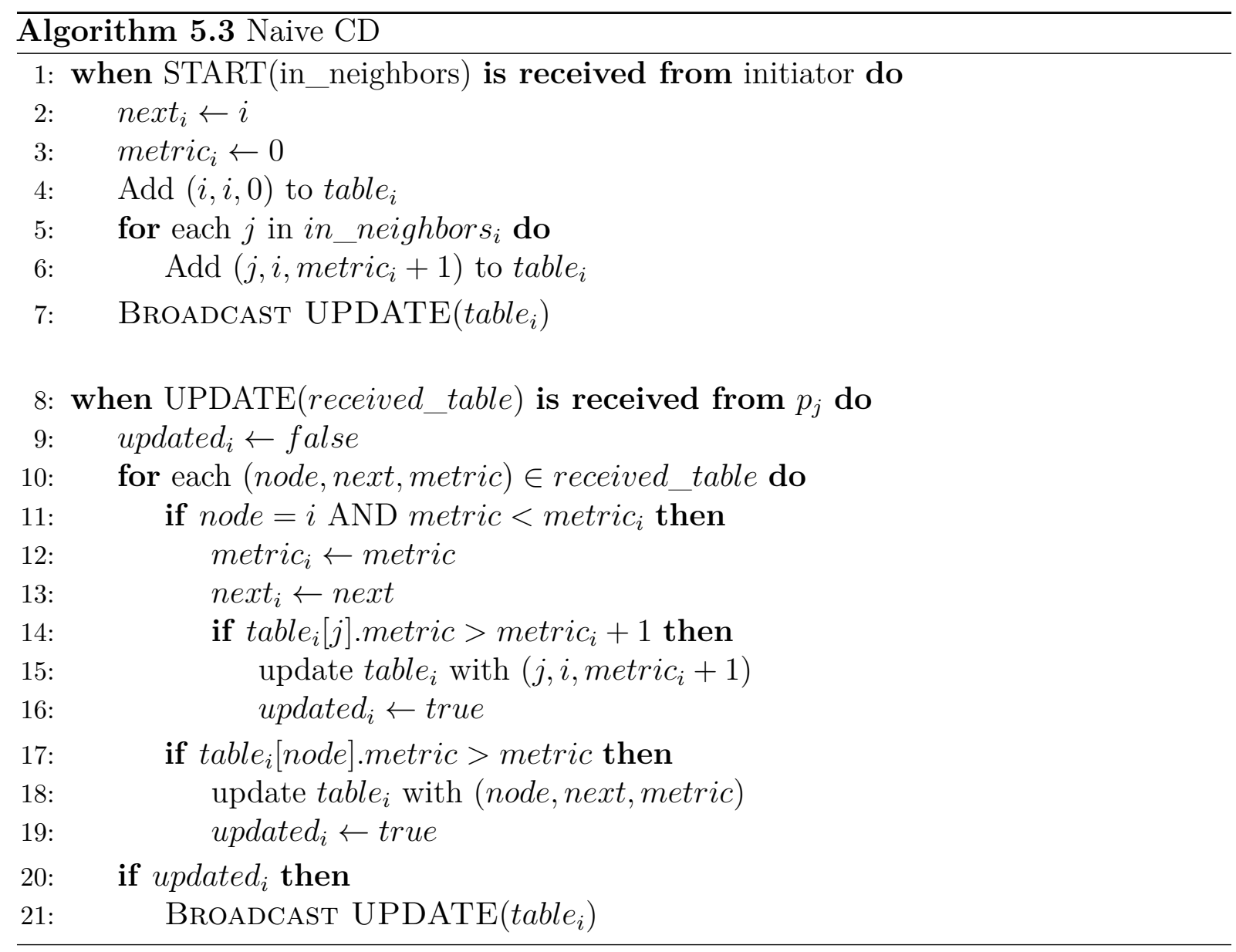


Inspired by Bellman-Ford algorithm, we propose an algorithm to perform controller discovery on directed graphs in Algorithm 5.3. Instead of discovering the shortest path between every pair of nodes in the graph, only the shortest path towards the controller is discovered. Each node in the network propagates the current best known distance between each node in the network and the controller, instead of propagating only their own cost and reachability information. For simplicity, this version of the algorithm assumes that there are no message losses and the directed graph is connected and constant.

A node $i$ maintains the following variables: next $t_{i}$, the next hop towards the controller; metric $c_{i}$, the route cost associated with sending a packet to the controller through next $_{i}$; table $_{i}$, a table whose lines are triples [node id, next hop towards the controller, associated cost], indexed by the node id.

The controller must start the algorithm and must know its inbound neighbors beforehand (line 1). First, the controller sets the cost to reach itself as 0 by initializing the value of $n e x t_{i}$ to itself, the value of metric $c_{i}$ to 0 , and adding this values to table $_{i}$ (lines 2-4). Next, the controller uses the inbound neighborhood information to populate table $_{i}$, that is, each inbound neighbor is able to reach the controller in one hop (lines 5 and 6 ). Finally, the table is broadcast in an UPDATE message (line 7). Note that, different from Bellman-Ford, we use the broadcast communication primitive, as the outbound neighbors are not assumed to be known.

A receiving node iterates over each entry of the table received in an UPDATE message. The node checks if the current entry is better than the currently stored path towards the controller (line 11). If so, the metric $c_{i}$ and next $t_{i}$ variables are updated with the values from the received table (lines 12 and 13). This update procedure may change the sender metric towards the controller, which is checked in lines 14-16. If the sender is not yet in the local table, its metric is assumed to be infinity. The node also checks if the received table entry is better than the estimate in the local table (lines 17-19). Lastly, the node propagates its current $t_{a b l e}$ if it was altered (lines 20 and 21). Eventually, all metric $_{i}$ and next $t_{i}$ variables converge to the optimal values.

For illustration purposes, consider a 3-node topology with the following links: Controller $(\mathrm{C}) \Rightarrow$ node 2 , node $2 \Rightarrow$ node 1 , node $1 \Rightarrow \mathrm{C}$. Table 17 shows the contents of table $_{i}$ for the controller, node 1 and node 2 . Each round represents a transmission of an UPDATE message (note that this is the only message order possible).

In the first round, the controller knows that node 1 can reach it and propagates this information to node 2 in round 2 . In round 3 , node 1 gets to know that it may reach the controller, as a consequence, the sender (node 2) may reach the controller through the receiver (node 1). This information is relayed to the controller in round 4, and Node 2 knows how to reach the controller in round 5 . At this point, all nodes are able to communicate with the controller. 
Table 17 - Simple Controller Discovery algorithm example with unidirectional links only

\begin{tabular}{c|c|c|c|c|c|c}
\hline \hline \multirow{2}{*}{ Round } & \multicolumn{2}{|c|}{ Controller } & \multicolumn{2}{c|}{ Node 1 } & \multicolumn{2}{c}{ Node 2 } \\
\cline { 2 - 7 } & Node & Next hop & Node & Next hop & Node & Next hop \\
\hline 1 & 1 & $\mathrm{C}$ & & & & \\
\hline 2 & 1 & $\mathrm{C}$ & & & 1 & $\mathrm{C}$ \\
\hline \multirow{2}{*}{3} & 1 & $\mathrm{C}$ & 1 & $\mathrm{C}$ & 1 & $\mathrm{C}$ \\
& & & 2 & 1 & & \\
\hline \multirow{2}{*}{4} & 1 & $\mathrm{C}$ & 1 & $\mathrm{C}$ & 1 & $\mathrm{C}$ \\
& 2 & 1 & 2 & 1 & & \\
\hline \multirow{2}{*}{5} & 1 & $\mathrm{C}$ & 1 & $\mathrm{C}$ & 1 & $\mathrm{C}$ \\
& 2 & 1 & 2 & 1 & 2 & 1 \\
\hline \hline
\end{tabular}

Source: author

Notice the algorithm also works on bidirectional links, since an UPDATE message sent to a bidirectional neighbor would immediately trigger a controller route discovery (unless a better route has been discovered previously). Consider a 3-node topology with the following links: Controller $(\mathrm{C}) \Leftrightarrow$ node 2 , node $2 \Leftrightarrow$ node 1 , node $1 \Rightarrow$ C. Table 18 shows the contents of table $_{i}$ for the controller, node 1 and node 2 . In the first round the controller knows nodes 1 and 2 can reach it directly. This information is transmitted to node 2 through the bidirectional link. In round 2, node 2 acquires this information, and is already able to reach the controller directly. Finally, in round 3, node 1 discovers from node 2 it can reach the controller directly through a unidirectional link, finishing the algorithm execution.

Table 18 - Simple Controller Discovery algorithm example with bidirectional links

\begin{tabular}{c|c|c|c|c|c|c}
\hline \hline \multirow{2}{*}{ Round } & \multicolumn{2}{|c|}{ Controller } & \multicolumn{2}{c|}{ Node 1 } & \multicolumn{2}{c}{ Node 2 } \\
\cline { 2 - 7 } & Node & Next hop & Node & Next hop & Node & Next hop \\
\hline \multirow{2}{*}{1} & 1 & C & & & & \\
& 2 & C & & & & \\
\hline \multirow{2}{*}{2} & 1 & C & & & 1 & C \\
& 2 & C & & & 2 & C \\
\hline \multirow{2}{*}{3} & 1 & C & 1 & C & 1 & C \\
& 2 & C & 2 & C & 2 & C \\
\hline
\end{tabular}

Source: author

The message cost of the proposed algorithm is also exponential in the worst case, a characteristic inherited from Bellman-Ford. We argue this flooding-like behavior is acceptable, since the controller discovery algorithm runs only at the network startup. At least in the case of a directed circle, the time complexity is quadratic (each non-controller node takes $n-1$ rounds to find the controller). The maximum length of an UPDATE message is proportional to the number of nodes in the network, considering that only the available information is transmitted. The same applies to the local memory cost of the algorithm. 


\subsubsection{Implementation details}

The simple controller discovery algorithm has the shortcomings of assuming a static network topology and assuming CD beacons are loss free. To work around these limitations, the original algorithm was modified three ways, as follows: (i) each entry of the reachability table is transmitted up to a certain number of times by each node; after this threshold, the entry is removed from the node memory, thus, lightening the burden on the memory usage. We chose three as the number of retransmissions as a trade-off between redundancy and memory usage; (ii) the implemented algorithm relies on the neighbor discovery algorithm to update the reachability table; and (iii) the implemented algorithm is controller-centric, in the sense that only the controller adds new values to the reachability table disseminated in the network.

Algorithm 5.4 summarizes what was actually implemented. Each node stores a reachability table with entries $\left(N_{A}, N_{B}, H_{c}\right)$, in which $N_{A}$ and $N_{B}$ are node identification values representing an existing edge in the network graph, and $H_{c}$ is an integer number representing the hop count from node $N_{A}$ to the controller. $N_{A}$ values within the table are unique. There is a retransmission counter associated with each entry, which controls the maximum number of times a node transmits this entry in a controller discovery beacon packet. Each table entry occupies 8 bytes of RAM memory, while it takes 5 bytes in the packet payload. We limit the reachability table size to 20 entries, all 20 entries fit in a single CD beacon packet.

Lines 1 and 2 sets global parameters used by the algorithm, the maximum number of retransmissions of a table entry, and the interval between table verification.

Lines 4-9 specifies the controller behavior when receiving a neighbor report packet from any node $\mathrm{j}$ in the network. Notice that this includes neighbor report packets from the controller itself. The controller uses its local network representation graph to calculate the shortest path length from each node $\mathrm{K}$ within the neighbor report packet to the controller (lines 5 and 6). If the distance from neighbor $\mathrm{K}$ to the controller is less than the currently stored value, the retransmission counter is set to MAX_RETRANSMISSION (lines 7-9).

Nodes process a controller discovery packet as described in lines 11 to 20. For each entry received within the CD packet, the receiver $i$ checks if the entry contains a better route for it to reach the controller. If so, the local variables next_hop $i$ and n__hops $i$ are updated and a neighbor report packet is sent to the controller (lines 13-16). The local reachability table is updated if the information from the received CD packet contains new or more efficient routes (lines 17-20).

Nodes and the controller periodically transmit CD packets as specified in lines 22-26. Note that the retransmission counter is decremented and a packet is transmitted only if there are valid entries in the local reachability table. Therefore, if the network 


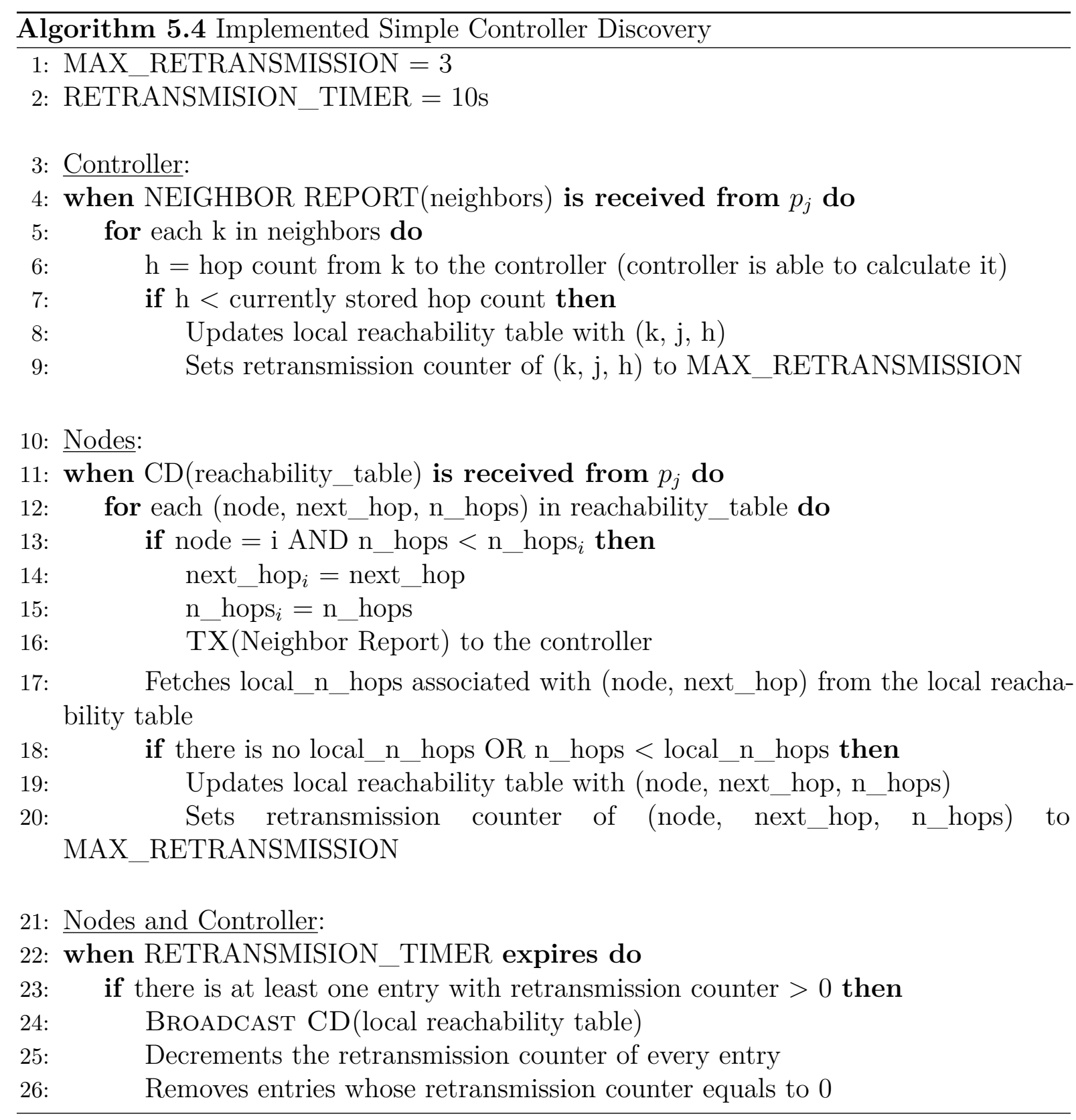

topology is stable, the controller discovery protocol ceases transmitting packets once all reachability information is disseminated.

\subsection{Enhanced Discovery Algorithms}

The algorithms presented in Section 5.1 serve the purpose of confirming SDN as a viable solution to routing on a network with unidirectional links. In this section, we enhance the neighbor discovery algorithm with mechanisms to curb its message overhead. Also, another controller discovery with a smaller memory footprint is proposed, considering a relaxed problem definition.

The content of this section was published at the $15^{\text {th }}$ Wireless On-demand Network systems and Services Conference (WONS) (ALVES; MARGI; KUIPERS, 2019). 


\subsubsection{Enhanced Neighbor Discovery}

The enhanced neighbor discovery keeps the same core principles of the simple neighbor discovery algorithm: asynchronous broadcast beacon packets and storing only the inbound neighbors.

Since beacon packets do not serve any other purpose and increase medium access congestion, we devised mechanisms to reduce the number of beacon packets transmissions. This is achieved at the expense of a small increase in algorithmic complexity by leveraging overhearing (Section 5.2.1.1) and non-constant beacon intervals (Section 5.2.1.2).

Also, new features are added to the discovery algorithm. In Section 5.2.1.3, we describe how to integrate the task of neighbor discovery with link quality estimation. Furthermore, since discovery algorithms often focus on adding nodes in the neighbor tables and neglect node departure detection, we describe a scheme for detecting node unreachability considering unidirectional links in Section 5.2.1.4.

A condensed algorithm description is provided in Section 5.2.1.5, while Section 5.2.1.6 contains implementation details.

\subsubsection{Neighbor discovery by overhearing}

Neighbor discovery by overhearing, also known as passive neighbor discovery, is an inexpensive way of detecting surrounding nodes (WOO; TONG; CULLER, 2003; BARRENETXEA et al., 2008). However it may yield inconsistent discovery delays and hinder node departure detection. Therefore, we propose to jointly use passive and active discovery. The purpose of beacon packets is to advertise the sender existence. However, this can be achieved by any broadcast packet, as long as the neighbor discovery protocol is informed of the reception and the addressing information is correct.

Unicast packets may also be used for discovery due to the broadcast nature of a wireless medium. However if the network uses a radio duty cycling protocol, unicast packets are unlikely to be overheard by all neighbors, thus unicast packets cannot be used as a substitute for ND beacons.

Relying solely on overhearing increases the uncertainty of the discovery delay, as there are no guarantees of packet transmission by other protocols or applications. To overcome this drawback, a node should send periodic beacons in the absence of other packet transmissions. To achieve the desired behavior, each node sets a timer to transmit a beacon packet according to the default interval. Every time any broadcast packet is transmitted, the timer is reset, postponing the beacon transmission. This ensures a minimum packet transmission rate, guaranteeing continuous discovery while avoiding unnecessary beacons. 


\subsubsection{Adaptive beacon interval}

Maintaining a constant beacon transmission rate is hardly the optimal strategy for saving network resources. A node should transmit more often at boot to enforce quick detection by peer nodes, while fewer packets may need to be transmitted when the network connections are stable. Therefore, a varying transmission interval decreases initial discovery delay and decreases the number of discovery packets.

The same way as the simple ND, the time of the first beacon transmission is randomized based on the node id, to avoid collisions. Every time a beacon is transmitted, the interval is doubled, up to a predefined maximum value.

Adaptive beacon transmission interval integrates almost seamlessly with overhearing. The caveat is that it may take longer to reach the maximum beacon interval, as the transmission timer is increased upon a beacon transmission, and the overhearing mechanism reduces the number of beacon transmissions.

\subsubsection{Link quality estimation (LQE)}

To the best of our knowledge, there has been hardly any work on link quality estimation over unidirectional links. Most packet reception ratio (PRR)-based estimators are based on acknowledged messages and calculate the metric at the transmitter (such as ETX (COUTO et al., 2005), F-ETX (BINDEL; CHAUMETTE; HILT, 2015), and EAR (KIM; SHIN, 2006)). An alternative to PRR-based estimators are the hardware-based estimators, such as LQI and RSSI. However, such estimators are hardware-dependent and inaccurate (BACCOUR et al., 2015).

ETF (Expected number of Transmissions over Forward links) estimates the delivery at the receiver by the ratio of received probe packets over the transmitted probe packets (SANG; ARORA; ZHANG, 2010). However, implementation details are not provided, for example, how a node knows the number of transmitted probe packets, what triggers a metric calculation, and how to estimate the time window.

We provide an LQE that estimates the link quality at the receiver and does not rely on link-layer acknowledgements, as ETF. Moreover, it does not rely on probes and employs a Moving Average algorithm similar to Woo and Culler (2003).

The receiver node maintains the status history (success or failure) of the last $n$ messages from each inbound neighbor. However, the history is updated only when successfully receiving a message, as lost messages are not detectable.

The number of lost messages between successful receptions are calculated according to sequence numbers. A sender maintains an individual sequence number counter for each outbound neighbor, plus one counter for the broadcast address. When a packet is received, receivers calculate the number of lost packets as the difference between the 
current sequence number and the previously received sequence number, minus one.

The link quality is estimated as the number of losses over the number of entries in the history. The loss rate is preferred over the success rate to provide an additive routing metric, that is, it is possible to calculate the cost of a given route by adding the metric values of the multiple links composing the route. The optimal route will be the one with the smallest cost, that is, with the least accumulated loss rate.

Figure 11 - Link quality estimation example.

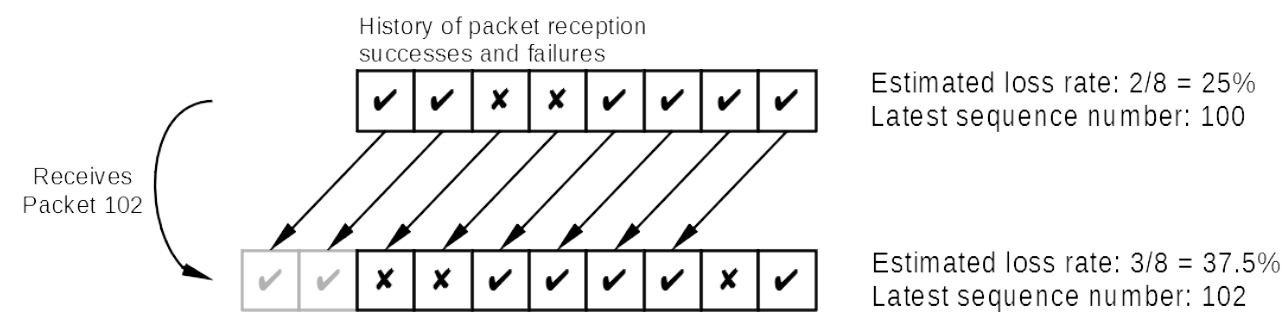

Source: author

Figure 11 shows an example of link quality estimation. In this case, the packet reception history size is 8 successes or failures. The estimated loss rate at the first situation is $\frac{2}{8}=25 \%$, since 2 out of the 8 last received packets were lost. The second situation represents the link quality after receiving a packet with sequence number 102 . The previous successfully received packet had sequence number 100, which means packet 101 was lost. Therefore, the oldest two entries in the reception history are deleted and two new entries are added: a failure and a success for packets 101 and 102, respectively. The new estimated loss rate is now $\frac{3}{8}=37.5 \%$

The history size is a key parameter, as it directly influences the estimator reactivity, stability and granularity. Also, in the context of Software-Defined Wireless Sensor Networking (SDWSN), LQE is also responsible for triggering the ND algorithm to send a neighbor report packet to the controller due to differences between the last reported link quality estimate and the current link quality estimate. We study how to set these parameters in Section 5.2.1.6.

\subsubsection{Node Unreachability Detection}

Node departure detection is a tricky task, since both false positives and false negatives can lead to route recalculations, decreasing the network packet delivery rate and energy efficiency.

If a node knows it is moving or its battery is low, it could send a message advertising this information to the neighborhood (active departure detection). However, devices are usually not provided with appropriate hardware to obtain such information. Also, the cause of the link failure is often oblivious to the node, e.g., due to environmental phenomena. 
Therefore, we focus on passive node departure detection. The periodic beacon transmission is the baseline for the detection, as it sets a minimum packet transmission rate.

A receiving node knows that at least one packet was lost if it has not received messages from a given neighbor for a time interval greater than the current beacon interval. As the beacon transmission interval is not constant, the interval must be included in the advertisement packets. A neighbor is removed from the neighbor table if it fails to deliver messages for a period longer than a multiple of the beacon intervals, the unreachability threshold $t$.

\subsubsection{Condensed Algorithmic description}

Algorithm 5.5 describes the enhanced neighbor discovery algorithm including all four added features in comparison to the simple algorithm: discovery by overhearing, adaptive beacon intervals, link quality estimation, and neighbor unreachability detection.

A node ${ }_{i}$ transmits beacons according to the transmission timer $\Delta t_{i}$ (lines 1 and 4 ). Adaptive beacon intervals are achieved by doubling the beacon transmission interval after each transmission, as shown in lines 2 and 3 .

Since discovery by overhearing is possible, any packet can trigger a neighbor discovery event. Therefore, every packet transmission resets the beacon transmission timer (lines 5 and 6).

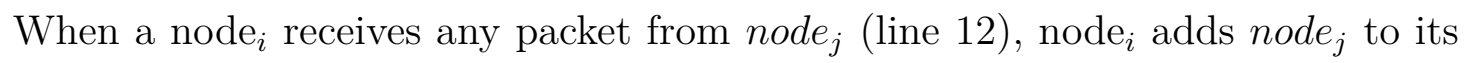
neighbor table (line 13), then updates the link quality estimation (lines 14-17), and, finally, resets information used for node unreachability detection: the lower bound estimate of lost packets loss_counter ${ }_{j}$ (line 19), and maximum tolerable number of beacon losses loss_threshold $($ line 20).

When a beacon packet is received, the same procedure triggered by any other packet reception is executed, with the addition of updating the unreachability timer, which is an information only present in beacon packets (lines 21-23).

A node ${ }_{j}$ is evicted from the neighbor table when the timer associated to node $_{j}$ expires loss_threshold $d_{j}$ times without receiving any packets from node $_{j}$, accomplishing node unreachability detection (lines 7-11).

\subsubsection{Implementation details}

The enhanced neighbor discovery algorithm takes on the tasks of link quality assessment (LQE), as described in Section 5.2.1.3, keeping the controller updated with the latest link quality information, and detecting neighbor departure. The LQE requires following parameters to be set: delivery success history size, threshold to send updated 


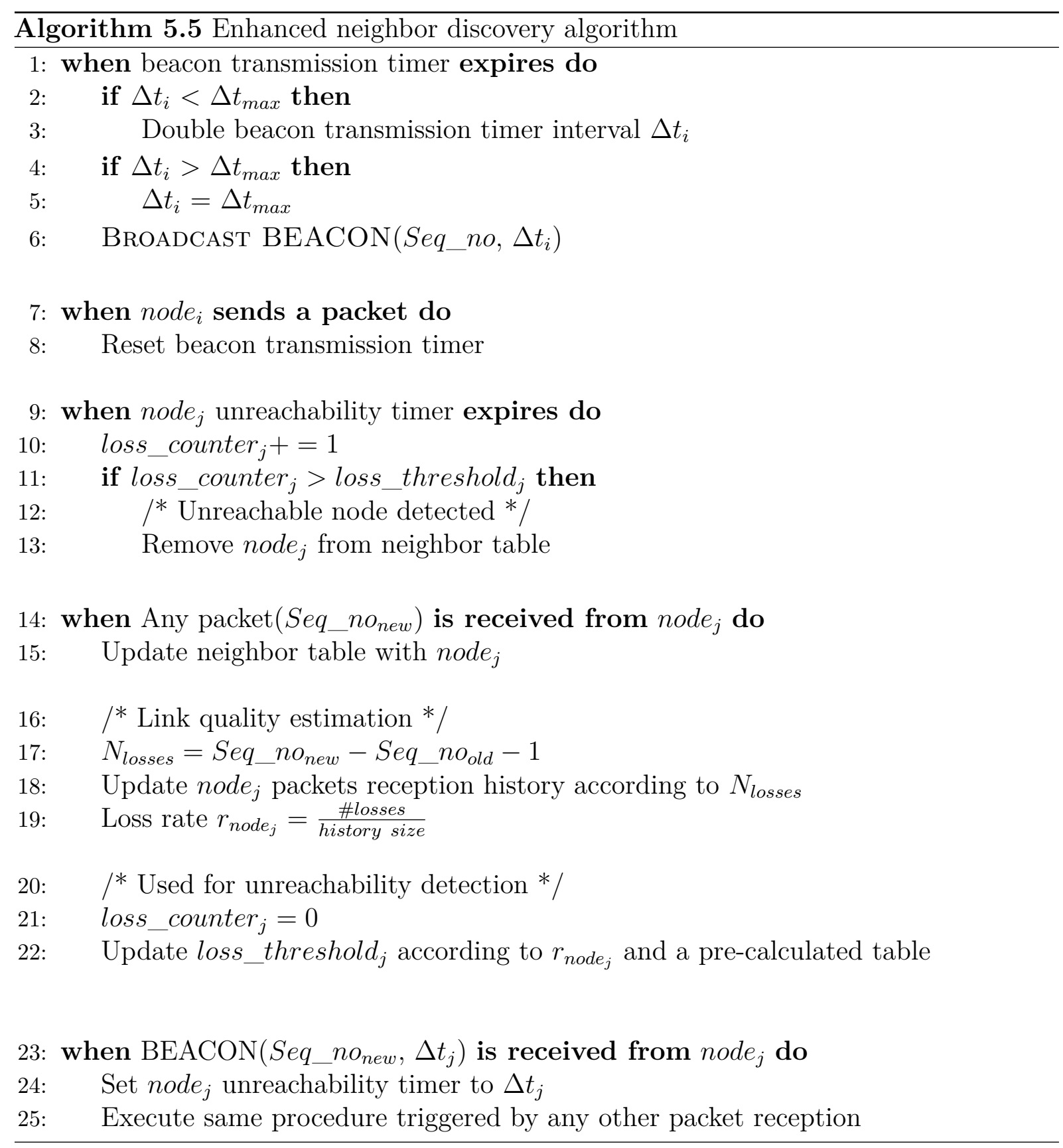

information to the controller, and neighbor departure detection threshold. We study and select these parameters below.

We have performed experiments to understand the effect of history size on the link quality estimation error and on the reactivity to changes in link quality. We have also studied the link quality variation threshold to send neighbor report updates to the controller. Both history size and update threshold are important parameters that directly impact the link quality estimation accuracy.

In this study, we model a communication channel by associating a success probability $p$ to it. A packet transmitted over the communication channel will be successfully delivered with probability $p$, that is, a packet transmission can be modeled as a random variable 
sampled from a Bernoulli distribution. Successive packet transmissions are considered independent.

The experiment consists in sampling the outcome of packet transmissions with different success probabilities, while estimating the LQE with different history buffer lengths. Table 19 shows the mean absolute error (MAE) and the maximum error obtained from filling a history buffer from a Bernoulli distribution with the given success probabilities $(p \in\{0.2,0.4,0.7,0.9\})$. We tested history sizes $(\mathrm{N})$ of 8,16 , and 32 , displaying the minimum error possible to each probability $p$, given the granularity provided by each history size. We draw 100k samples in each experiment, the MAE was calculated from all samples, while the maximum error was assessed only after filling the history buffer. In other words, the error between the current success probability estimation and the actual probability is calculated after each sample; the MAE is the average of all errors of all 100k samples, while the maximum error is largest error disregarding the first $\mathrm{N}$ estimates.

Table 19 - Influence of history size $(\mathrm{N})$ on LQE metrics.

\begin{tabular}{|c|c|c|c|c|c|}
\hline & \multirow[b]{2}{*}{$\mathrm{N}$} & \multicolumn{4}{|c|}{ Success probability $(p)$} \\
\hline & & 0.2 & 0.4 & 0.7 & 0.9 \\
\hline \multirow{3}{*}{$\begin{array}{l}\text { Minimum } \\
\text { error possible }\end{array}$} & 8 & 0.05 & 0.025 & 0.05 & 0.025 \\
\hline & 16 & 0.05 & 0.025 & 0.0125 & 0.025 \\
\hline & 32 & 0.0125 & 0.00625 & 0.0125 & 0.00625 \\
\hline \multirow{3}{*}{ MAE } & 8 & 0.131 & 0.144 & 0.131 & 0.084 \\
\hline & 16 & 0.086 & 0.101 & 0.114 & 0.060 \\
\hline & 32 & 0.059 & 0.069 & 0.073 & 0.042 \\
\hline \multirow{3}{*}{$\begin{array}{l}\text { Maximum } \\
\text { error }\end{array}$} & 8 & 0.675 & 0.600 & 0.575 & 0.650 \\
\hline & 16 & 0.487 & 0.537 & 0.387 & 0.338 \\
\hline & 32 & 0.331 & 0.350 & 0.325 & 0.275 \\
\hline \multirow{3}{*}{$\begin{array}{l}\text { MAE to last } \\
\text { sent value }\end{array}$} & 8 & 0.137 & 0.137 & 0.139 & 0.061 \\
\hline & 16 & 0.083 & 0.098 & 0.091 & 0.047 \\
\hline & 32 & 0.057 & 0.076 & 0.065 & 0.048 \\
\hline \multirow{3}{*}{$\begin{array}{l}\text { Updates triggered } \\
\text { per } 100 \text { samples }\end{array}$} & 8 & 25.31 & 21.10 & 12.14 & 2.69 \\
\hline & 16 & 11.58 & 10.47 & 5.79 & 1.12 \\
\hline & 32 & 7.24 & 6.22 & 3.41 & 0.85 \\
\hline
\end{tabular}

Source: author

As expected, a larger history yields less errors: doubling the history size causes a reduction of approximately $30 \%$ in the mean absolute error. The maximum error also tends to decrease with a larger history size, although not as consistently as the MAE (between $10 \%$ and $48 \%$ reduction).

In the context of SDWSN, the neighbor discovery algorithm is responsible for keeping the controller up-to-date with the link qualities in the network. The decision to send an update packet to the controller is based on the difference between the last reported link quality estimate and the current estimate. The controller is updated if the difference exceeds a certain threshold. A larger threshold results in less packets sent to the 
controller, at the cost of requiring more data to achieve a significant change in the link quality estimation. Therefore, there is a trade-off between control overhead and information freshness.

We analyze this trade-off by studying binomial distribution properties. The parameters of a binomial distribution are the success probability $p$ and number of trials $n$. The distribution is defined for integer values $k \in[0, n], k$ is the number of successes out of $n$ trials. Considering $p=50 \%$, as it yields the largest variance for binomial distributions (worst case), we calculate the values $k_{1}, k_{2}$ symmetrical to the distribution average $\left(p n=\frac{n}{2}\right)$, such that their probabilities sum up to $80 \%$. In other words, we find $j$ such that $k_{1}=\frac{n}{2}-j$, $k_{1}=\frac{n}{2}+j$, and $\operatorname{Pr}\left(k_{1} \leq k \leq k_{2}\right) \leq 80 \%$. The rationale is to trigger an update message only if the newly assessed packet delivery probability is unlikely to occur considering the previously sent estimate. Considering the binomial distribution is concentrated around the average, we chose the $80 \%$ arbitrarily as a trade-off between accuracy and stability (a threshold of $100 \%$ would never trigger an update message, while a threshold of $0 \%$ would trigger an update message whenever two consecutive estimates are different).

This calculation gives $\left(k_{1}, k_{2}\right)=(3,5)$ for $n=8,\left(k_{1}, k_{2}\right)=(6,10)$ for $n=16$, and $\left(k_{1}, k_{2}\right)=(13,19)$ for $n=32$, representing a threshold of 12.5 percentage points for $n=8$ or $n=16$, and 9.375 percentage points for $n=32$.

Based on these thresholds, the mean absolute error to the last transmitted value is measured as exhibited in Table 19. We observe that the error is in the same order of magnitude as the local mean error. Also in the table, we show the number of updates triggered at every 100 samples, which can be interpreted as the amount of unnecessary neighbor reports caused by statistical noise. The amount of reports is larger for low delivery probability and for smaller $n$.

Table 20 shows experiments regarding the reactivity by changing the success probability during the sampling experiment. The crossing value represents the average number of trials until the reported value is within $12.5 \%$ of the new probability, while the first report represents the average number of trials until a neighbor report is issued.

The crossing value is smaller when the success probability suffers little variations in comparison to when large variations occur. The reason is that the last reported value is probably already close or even already within the $12.5 \%$ range of the new probability.

If the probability changes abruptly (e.g. transition $0.2 \rightarrow 0.9$ ) the results show that the history buffer needs to be overwritten to achieve a good estimation. On the other hand, the first report occurs quicker, meaning that the estimator detects a change in the probability, but avoids abrupt changes in the estimation.

Observing all values, we chose 16 as history size as the experiments indicate a balance between accuracy and reactivity. 
Table 20 - Reactivity study.

\begin{tabular}{|c|c|c|c|c|c|}
\hline & \multirow[b]{2}{*}{$\mathrm{N}$} & \multicolumn{4}{|c|}{ Transitions } \\
\hline & & 0.9 to 0.8 & 0.8 to 0.9 & 0.3 to 0.2 & 0.2 to 0.3 \\
\hline \multirow{3}{*}{$\begin{array}{l}\text { Crossing } \\
\text { value }\end{array}$} & 8 & 8.5 & 3.6 & 15.2 & 10.8 \\
\hline & 16 & 10.2 & 4.4 & 19.2 & 7.7 \\
\hline & 32 & 6.0 & 6.8 & 14.4 & 7.0 \\
\hline \multirow{4}{*}{$\begin{array}{l}\text { First } \\
\text { report }\end{array}$} & 8 & 10.3 & 10.8 & 17.4 & 10.0 \\
\hline & 16 & 20.6 & 23.5 & 29.4 & 16.2 \\
\hline & 32 & 36.2 & 45.5 & 44.7 & 23.8 \\
\hline & & 0.9 to 0.2 & 0.2 to 0.9 & 0.6 to 0.4 & 0.4 to 0.6 \\
\hline \multirow{3}{*}{$\begin{array}{l}\text { Crossing } \\
\text { value }\end{array}$} & 8 & 17.7 & 7.7 & 9.6 & 4.8 \\
\hline & 16 & 25.5 & 13.8 & 13.2 & 7.4 \\
\hline & 32 & 36.3 & 26.9 & 20.7 & 16.0 \\
\hline \multirow{3}{*}{$\begin{array}{l}\text { First } \\
\text { report }\end{array}$} & 8 & 7.8 & 2.3 & 8.5 & 5.0 \\
\hline & 16 & 9.1 & 3.2 & 15.5 & 9.2 \\
\hline & 32 & 10.8 & 4.2 & 20.4 & 14.4 \\
\hline
\end{tabular}

Source: author

Regarding neighbor unreachability detection, the unreachability threshold $t$ is the number of beacon intervals elapsed since the latest received packet that triggers a neihgbor removal from the neighbor table. The threshold $t$ is precalculated based on the current estimated loss rate $r$ (provided by the LQE) as the minimum $t$ such that $r^{t} \leq$ max $_{n e g}$, in which $\max _{n e g}=1 \%$ is the maximum rate of false negatives.

Table 21 - Loss threshold to remove a neighbor from the neighbor table.

\begin{tabular}{ccc}
\hline \hline Loss rate estimate & Threshold $t$ & False negative rate \\
\cline { 2 - 4 } $0.00 \%$ & 2 & $0.00 \%$ \\
$6.25 \%$ & 2 & $0.39 \%$ \\
$12.50 \%$ & 3 & $0.20 \%$ \\
$18.75 \%$ & 3 & $0.66 \%$ \\
$25.00 \%$ & 4 & $0.39 \%$ \\
$31.25 \%$ & 4 & $0.95 \%$ \\
$37.50 \%$ & 5 & $0.74 \%$ \\
$43.75 \%$ & 6 & $0.70 \%$ \\
$50.00 \%$ & 7 & $0.78 \%$ \\
$56.25 \%$ & 8 & $1.00 \%$ \\
$62.50 \%$ & 8 & $2.33 \%$ \\
$68.75 \%$ & 8 & $4.99 \%$ \\
$75.00 \%$ & 8 & $10.01 \%$ \\
$81.25 \%$ & 8 & $18.99 \%$ \\
$87.50 \%$ & 8 & $34.36 \%$ \\
$93.75 \%$ & 8 & $59.67 \%$ \\
\hline \hline
\end{tabular}

Source: author 
We also adopt 2 as the minimum value of $t$ to avoid false positives, while the maximum value is 8 to avoid delaying the departure detection for too long. Threshold $t$ values and the corresponding false negative rates are shown in Table 21, considering all link quality estimation possibilities of a 16-bit estimation.

\subsubsection{Enhanced Controller Discovery}

The controller discovery problem is inherent to SDWSN and requires a global algorithm to solve the general unidirectional link case. For example, a unidirectional circle topology, such as the one illustrated in Figure 12a, requires a global algorithm. In this particular example, the only way node 7 may know it reaches the controller directly is propagating this information via node 1 throughout node 6 , i.e., all nodes must know this information at some point of the algorithm execution.

Figure 12 - Examples of topologies with unidirectional links:

$$
\begin{aligned}
& \text { (b) Network with unidirectional } \\
& \text { links and a bidirectionally con- } \\
& \text { nected component. }
\end{aligned}
$$

(a) Unidirectional circle.
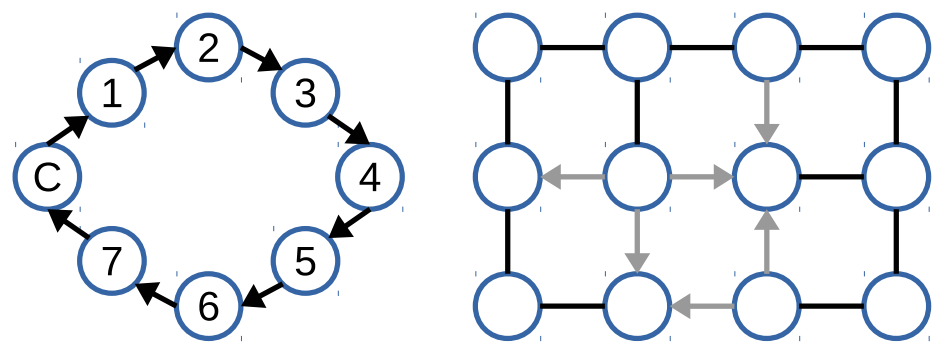

Source: author

If the network graph contains a bidirectionally connected component, that is, the topology graph is still connected if all unidirectional links are removed, as the example in Figure 12b, then controller discovery can be solved by a local algorithm. We believe it takes very specific radio and environmental conditions to result in a pure unidirectional network. Therefore, the existence of a bidirectionally connected component is plausible in practice.

With this assumption in mind, building a tree rooted at the controller provides an efficient solution to the problem, although nodes out of the bidirectionally connected component, if any, would not be able to join the network.

Algorithm 5.6 provides such an algorithm. The algorithm starts by receiving the inbound neighborhood information from the neighbor discovery algorithm (line 1). Next, the route cost is initialized with the minimum hop count value at the controller, while other nodes are initialized with the maximum hop count value (lines $2-5$ ). The initialization is concluded by broadcasting a controller discovery packet (CD_PACKET) containing the inbound neighborhood and the route cost towards the controller (line 6). 


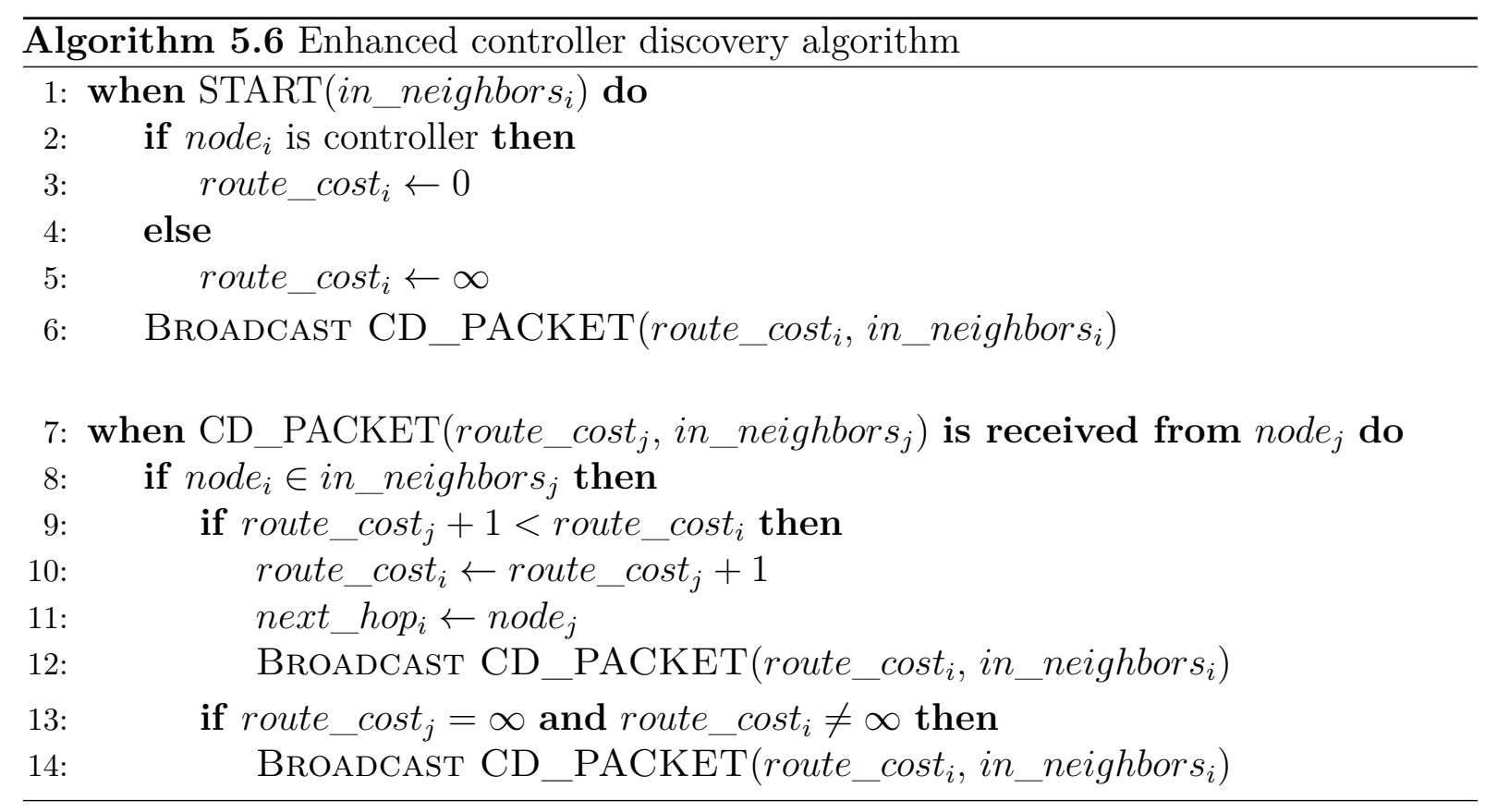

When a controller discovery packet is received (line 7 ), the receiving node $_{i}$ verifies if the link between node $_{i}$ and node ${ }_{j}$ is symmetric by checking whether node inbound

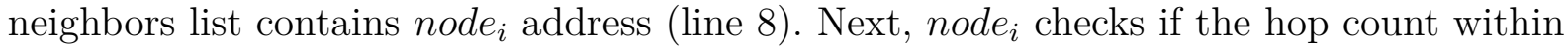
the packet is smaller than the currently stored hop count value. If both conditions are true, the next hop towards the controller and the hop count value are updated and the new route discovery is informed to the SDN layer (lines 9-11). Also, a controller discovery packet is scheduled for transmission (line 12).

Since the route set by the controller discovery algorithm is temporary, the algorithm does not seek the optimal route in terms of link quality and relies on hop count to build the tree. Also notice this algorithm does not contain configurable parameters.

A node $_{i}$ also transmits a controller discovery packet whenever it already knows how to reach the controller and receives a controller discovery packet with the maximum metric from another node node $_{j}$ (lines 13 and 14). This condition indicates node still does not know how to reach the controller and node $_{i}$ is a relay node candidate. This procedure is intended to speed up controller discovery by nodes joining the network after the initial network bootstrap.

Although an individual node does not know whether the other nodes have already obtained a valid route towards the controller, the controller discovery algorithm eventually stops sending messages if the network topology is stable and the nodes' neighborhoods remain constant.

Algorithm 5.6, as it is written, does not deal with changing neighborhoods. In an actual implementation, a controller discovery packet should be transmitted whenever a neighbor is added to the inbound neighborhood. 
Figure 13 - Sample network to controller discovery.

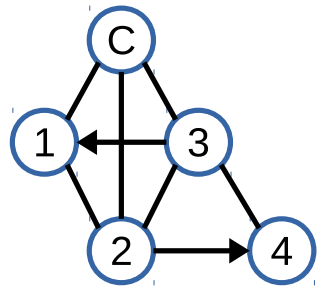

Source: author

Take the network of Figure 13 and the execution trace in Table 22 as an example. First, the variables are initialized in Round 1, i.e., the controller has cost 0 and the other nodes infinity. Next, the controller detects nodes 1, 2, and 3 as inbound neighbors and transmits a controller discovery beacon with this information. As the links are bidirectional, these nodes can reach the controller directly (Round 2).

In the next round, nodes 1, 2, and 3 transmit their own beacon with neighborhood information. Node 2 does not switch the next hop to 1 or 3 , because it is a longer route. Analogously, node 1 does not switch the next hop to 3 due to link being unidirectional. Node 4 sets the next hop as node 3 , since the beacon received from node 2 does not contain its address (unidirectional link). Node 4 also transmits a beacon to advertise its discovery, but it has no effect on the current routes.

At the end of Round 3, the algorithm has converged and controller discovery beacons are no longer transmitted because (i) the topology is stable and the set of neighbors does not change, and (ii) none of the nodes will change their next hop towards the controller.

Table 22 - Enhanced Controller Discovery algorithm example

\begin{tabular}{c|c|c|c|c|c|c|c|c|c|c}
\hline \hline \multirow{2}{*}{ Round } & \multicolumn{2}{|c|}{ C } & \multicolumn{2}{|c|}{1} & \multicolumn{2}{c|}{2} & \multicolumn{3}{|c|}{3} & \multicolumn{2}{|c}{4} \\
\cline { 2 - 12 } & Next & Cost & N & C & N & C & N & C & N & C \\
\hline 1 & C & 0 & - & $\infty$ & - & $\infty$ & - & $\infty$ & - & $\infty$ \\
\hline 2 & C & 0 & $\mathrm{C}$ & 1 & $\mathrm{C}$ & 1 & $\mathrm{C}$ & 1 & - & $\infty$ \\
\hline 3 & $\mathrm{C}$ & 0 & $\mathrm{C}$ & 1 & $\mathrm{C}$ & 1 & $\mathrm{C}$ & 1 & 3 & 2 \\
\hline \hline
\end{tabular}

Source: author

\subsubsection{Implementation details}

The implementation of the enhanced controller discovery algorithm (Section 5.2.2) was straightforward, using Contiki's abstractions to store data and to transmit CD beacons.

\subsection{Radio duty Cycling}

A solution for routing over unidirectional links would not be complete without a Radio Duty Cycling (RDC) protocol. Energy efficiency is a key performance indicator 
for WSN and IoT. Since the main source of energy consumption in such networks is the radio (SHIBATA et al., 2016), radio duty cycling mechanisms have been devised to reduce the amount of time the transceiver is active (i.e., in transmitting or receiving states).

RDCs are classified as either synchronous or asynchronous. Synchronous protocols rely on Time Division Multiple Access (TDMA) and require a setup phase to compute the transmission schedule. Conversely, asynchronous RDCs operate without prior scheduling and do not require tight global clock synchronization.

Most existing asynchronous RDCs do not support unidirectional links properly, as they heavily rely on link layer acknowledgements. In this section, we provide an asynchronous RDC which is able to employ energy saving techniques on bidirectional and unidirectional links. The contribution presented in this section was published at Elsevier Computer Communications journal (ALVES; MARGI; KUIPERS, 2020).

We focus on adapting asynchronous RDCs for unidirectional links. Preamble sampling is a basic technique in which the sender transmits a preamble with the objective of warning the receiver of the upcoming packet transmission. Receivers, in turn, periodically check the medium for ongoing transmissions.

ContikiMAC (DUNKELS, 2011) enhances the basic preamble sampling technique, reducing the preamble duration and, consequently, reducing the overall energy consumption. The main techniques employed by ContikiMAC are preamble packetization, early acknowledgement, and phase lock.

Figure 14 - Examples of preamble sampling techniques. ContikiMAC uses techniques to reduce energy footprint.

(a) Long preamble RDC

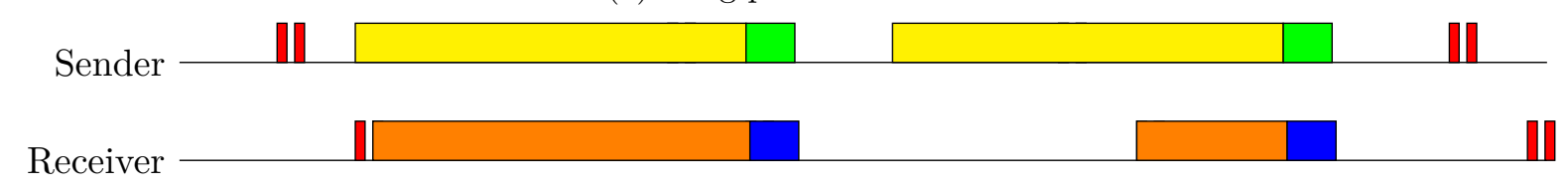

(b) ContikiMAC

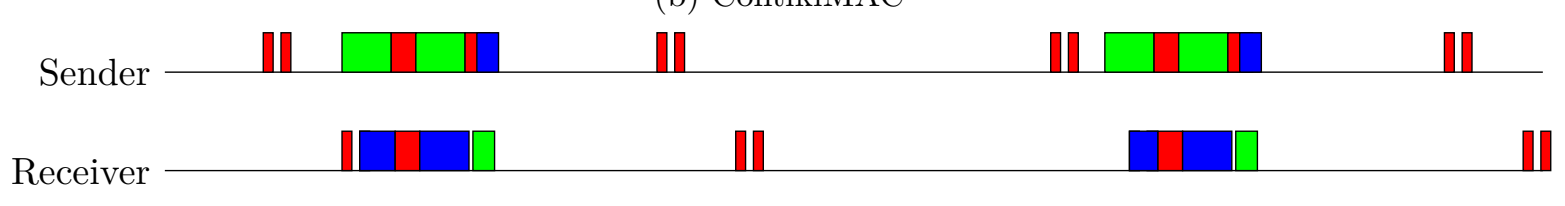

(c) Color code

Idle listening $\square$ Sending preamble $\square$ Receiving preamble $\square$ Sending data $\square$ Receiving data

Source: author

Preamble packetization consists of strobing multiple copies of the packet instead of transmitting a long preamble without useful information. Packetization enables the receiver to send an early acknowledgement, thus, shortening the preamble stream. In addition, if 
phase lock is used, the sender registers the time at which it received the acknowledgement, calculates the phase shift between sender and receiver wake-up times, and postpones the start of preamble transmission to the moment the receiver is about to start listening.

Figure 14 exemplifies packet transmissions with two RDCs: a simple preamble sampling protocol with long preambles and ContikiMAC. The beginning of each packet transmission (from an upper layer in the protocol stack) and the node wake up instants are the same in Figures 14a and 14b.

Considering the long preamble RDC, each node periodically checks the medium for transmissions two times in succession (red shade in Figure 14a). The frequency the nodes check the medium is called channel check rate. A transmitter always transmits the preamble full length (shaded in yellow), followed by the data packet (green shade). The amount of time the receiver stays awake before receiving the actual data packet depends on the moment it detects the preamble. In the first packet transmission exemplified, the receiver detects the preamble early on, staying awake nearly the whole preamble duration (orange shade). In the second transmission, the receiver stays awake less than half of the preamble, after which the data is received (blue shade).

In Figure 14b we investigate the same packet transmission situations if ContikiMAC is used. Notice there is no yellow and orange shades, since the preamble packetization technique transforms the preamble into a repetition of (the same) data packet. The first repetition of the first packet transmission awakes the receiver, which maintains its radio on until receiving the full packet in the second repetition. The receiver then transmits an early acknowledgment (short green shade), which informs the sender the transmission was successful. Consequently, the first packet transmission terminates earlier in ContikiMAC in comparison to the long preamble algorithm.

After receiving the acknowledgement, the transmitter estimates the time the receiver awakes (in comparison to its own wake-up schedule) as part of the phase lock mechanism. In the second packet transmission, the sender uses this information to start the transmission on a later moment, in comparison to the long preamble RDC. Therefore, in the second packet transmission, we can observe all of ContikiMAC mechanisms in action (preamble packetizaition, early ACK, and phase lock) to reduce the preamble length. In other words, it is noteworthy that both transmitter and receiver radios are active for a shorter amount of time in ContikiMAC in comparison to the long preamble technique.

However, ContikiMAC's improvements rely on the existence of a bidirectional link; without the receiver sending acknowledgements, the improvements do not work.

We leverage the centralized control provided by SDN to work around the lack of acknowledgements to enable ContikiMAC techniques over unidirectional links. We add a timestamp to the packets, so the receiver is able to calculate the wake-up phase shift from 
the sender. The receiver informs the controller about the phase shift, which, in turn, informs the sender. In the next transmission, the sender transmits only the preamble packets at the moment the receiver is expected to be listening. Figure 15a shows a transmission before the phase lock, in which the packet is strobed throughout the whole listening interval. Packet number 3 is received, and the receiver informs the controller about the calculated phase shift. In Figure 15b, the sender is already aware of the phase shift, and transmits only packet number 2 , which works as a wake-up tone, and packet number 3 , which is the one actually received.

Figure 15 - Unidirectional RDC: the controller informs the sender when it should start preamble transmission.

(a) Previous to phase lock

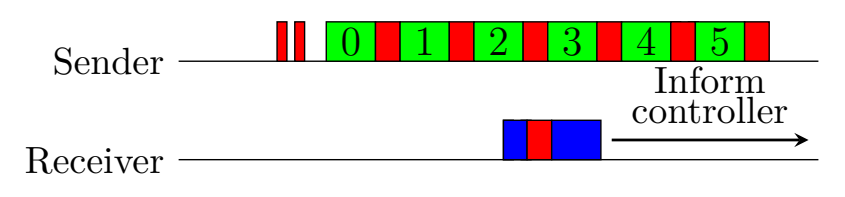

(b) After phase lock

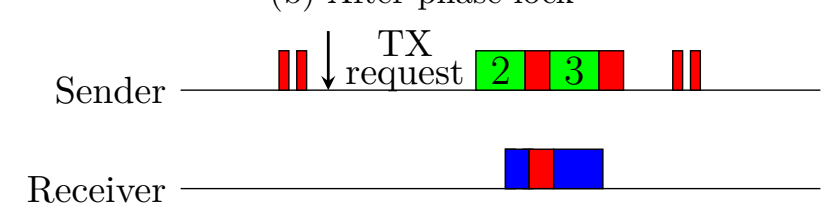

Source: author

The timestamp value encoded in the strobed packets is the time elapsed since the sender last woke up for medium checking. The receiver is only able to register the moment a packet is successfully received, thus, the phase shift PS is calculated as $P S=T_{s}-\left(T_{r}-\Delta p\right)$, where $T_{s}$ is the time stamped in the received packet (in the sender time base), $T_{r}$ is the end-of-reception time in the receiver and $\Delta p$ is the packet duration in milliseconds, as illustrated in Figure 16.

Figure 16 - Unidirectional RDC detailed timing diagram.

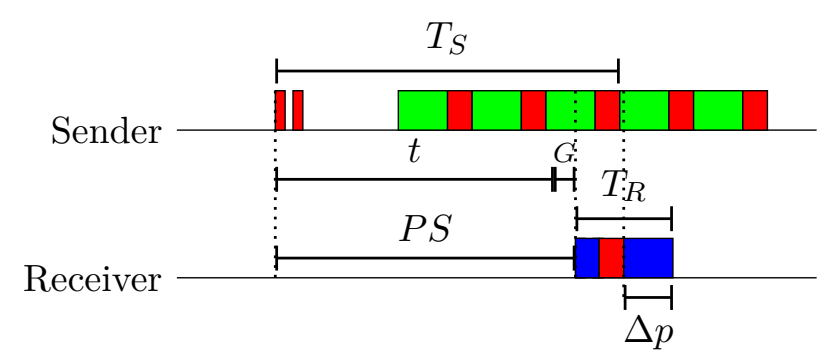

Source: author

In subsequent transmissions, the sender uses the phase shift information to transmit the first packet (wake-up tone) at the moment the receiver is checking the medium for transmissions. The sender transmits at time $t=P S-G$, as shown in Figure 16. $G$ represents a guard time, chosen as half of the packet length plus the time a node takes to check 
the medium for clearance. Mathematically, $G=\frac{\Delta p+2 * C C A+\triangle C C A}{2}$, where CCA is the time it takes to perform a clear channel assessment and $\triangle C C A$ is the time between two consecutive CCAs.

This choice of transmission time increases robustness against clock drifts, since, if the drift is zero, the receiver will wake up in the middle of the probe packet. Therefore, considering the clock drift might be positive or negative, this yields the optimal transmission time to prevent missing the packet transmission due to small drifts. If the receiver detects that the phase shift changed to an extent that is threatening successful packet transmissions, it should send the updated phase shift to the controller or directly to the sender. The phase shift update is triggered when the difference between the calculated value and last informed phase shift exceeds a given threshold. Complementarily, the sender could use successive phase shift updates to estimate the clock drift and automatically recalculate the current phase shift estimation, but this feature is not included in our implementation.

If the clock drift is too large, the receiver may miss packets from the sender, which would not be aware of the problem due to the lack of link-layer acknowledgements. Figure 17 exemplifies two cases of large drift, represented as $\delta$ in the picture. If the clock drift causes the receiver to wake up before the packet is in the air, the transmission fails (the example shows a drift $\delta=-\Delta p$ ). Analogously, if the receiver wakes up too late (the positive drift exemplified in the picture is $\delta=2.5 \Delta p$ ), the packet would not be received.

One way to alleviate this issue is to include timestamps in broadcast packets, for which the early acknowledgement and phase lock techniques are not used. Therefore, broadcast packets can be used for resynchronization.

Figure 17 - Example of packets missed due to large clock drift.

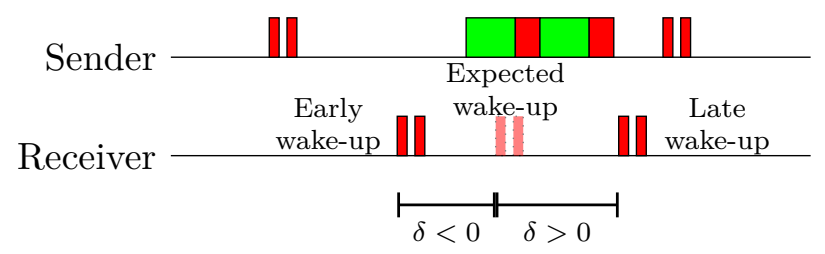

Source: author

If too many packets are lost, the sender will eventually be removed from the receiver's neighbor table. Consequently, the controller will instruct the sender to no longer use the receiver as a relay.

Note that, (i) in the case of communication over bidirectional links, the RDC operates as the original ContikiMAC protocol; and (ii) our RDC, like the ContikiMAC protocol, does not address the hidden node issue. 


\subsubsection{Implementation details}

The radio duty cycling algorithm deals with low level radio interactions and requires precise timing to work correctly. This section contains implementation details, and some modifications, to improve the performance of the unidirectional RDC deployment.

The radio driver in Contiki OS provides a function to load packets in the radio memory and a function to actually transmit a previously loaded packet. In the original ContikiMAC implementation, a packet is loaded to the radio only once before a sequence of strobe transmissions, while the transmit function is called repeatedly. However, the unidirectional RDC requires to load the packet before every strobe transmission, since the phase value has to be updated in the transceiver's memory.

The load function takes a non-negligible amount of time, therefore it is not feasible to load the packet immediately before the packet transmission while guaranteeing the timing constraints of the protocol. The workaround is to load the packet to the radio right after issuing a transmission. As a consequence, the packets contain the phase of the previously transmitted strobe packet, and the time calculations have to be adjusted accordingly.

The precision of the phase shift needs to be at least 0.1 milliseconds, since a 50-byte packet (minimum ContikiMAC packet size, inherited by our algorithm) takes approximately $1.6 \mathrm{~ms}$ to be transmitted. If the precision requirements are not met, the calculated phase shift value could contain a systematic error large enough to disturb protocol execution. In our implementation, we use msp430 clock ticks to measure the phase shift, which provides a precision of $1 / 32768 s \approx 0.03 \mathrm{~ms}$.

The packets containing phase information are not provided with end-to-end reliability. To make sure the phase information has reached its final destination, the sender uses a bit in the packet header to inform the receiver that it knows the phase. The receiver retransmits the phase information if it receives any non-acknowledgement-demanding unicast packet without the known phase bit set.

To increase robustness against clock drifts, we use the standard ContikiMAC guard time $(\approx 16 \mathrm{~ms})$ to send more strobe packets instead of only two (the originally designed wake-up tone and the received packet). The actual quantity of extra strobe packets depends on the packet size.

\subsection{Chapter summary}

We provided five algorithms that support unidirectional links. First, simple controller discovery and neighbor discovery algorithms were designed, as a proof of concept to using SDN to route through unidirectional links. These algorithms were called "simple" as 
they do not focus on performance, but provide basic functionality that allows the controller to build an accurate network representation.

Next, with the goal of creating efficient protocols, we created the enhanced controller discovery and neighbor discovery algorithms. These algorithms contain mechanism to reduce the overall control overhead and are expected to present better performance results.

The enhanced discovery algorithms are capable of tracking the status of links throughout the network lifetime. As a consequence, these algorithms are able to detect links that cease to exist or are formed due to node mobility. In other words, they should support node mobility, as long as the movement speed is not faster than the algorithms capability to detect link changes. The same argument applies to other topology changing situations, such as nodes that run out of energy and leave the network, or nodes that join the network after the initial setup.

Finally, we designed a Radio Duty Cycling protocol, given the lack of protocols in this category that embrace unidirectional links. We enhance the ContikiMAC protocol to create an asynchronous RDC, using SDN features to forego link layer acknowledgements. 


\section{Experiments}

This chapter contains the performance evaluation of the proposed algorithms. The experimental method is described in Section 6.1, including scenarios, tools, and evaluation metrics. Next, we present and discuss the obtained results in Section 6.2. The conclusions drawn from the experiments are summarized in Section 6.3.

\subsection{Method}

We designed experiments towards answering the question, "What are the gains obtained from exploring unidirectional links in low-power wireless networks?". To this end, we tested combinations of discovery algorithms and radio duty cycling algorithms under several topologies with and without unidirectional links.

First, we analyzed a set of simulations using a pure CSMA/CA medium access scheme (i.e., without duty cycling), aiming to assess the impact of exploring unidirectional links without the extra overhead imposed by an RDC. We compared a traditional discovery algorithm used in the literature, namely the Collect-based discovery (ALVES et al., 2019), to three versions of our discovery algorithms: (i) the simple version, (ii) the enhanced version using unidirectional links, and (iii) the enhanced version blacklisting unidirectional links. This set of experiments is displayed in Section 6.2.1.

The second set of experiments studied the influence of radio duty cycling on exploring unidirectional links. We paired the simple version of our discovery algorithm to the baseline version of our RDC. Next, the enhanced discovery algorithms was combined with two versions of our RDC protocol: (i) the baseline version, in which the phase information is directly sent to the target node, and (ii) a modified version, in which the phase information is always sent to the controller. As a comparison, we instructed the controller to calculate only bidirectional paths and use the original ContikiMAC RDC protocol combined with our enhanced discovery algorithms. Finally, as a baseline, we also combined the Collect-based discovery protocol with ContikiMAC RDC. We used a channel check rate, i.e., the frequency the nodes check the medium, of $8 \mathrm{~Hz}$ in all cases. Experiments with RDC are discussed in Section 6.2.2.

Table 23 summarizes the combinations of discovery algorithms and radio duty cycling algorithms used in the performance evaluation.

It would be interesting to test the performance of non-SDN solutions, such as BRA (RAMASUBRAMANIAN; MOSSE, 2008) or ULC (KARNAPKE; NOLTE, 2015); however, there is no implementation of such algorithms readily available. 
Table 23 - Combinations of algorithms tested.

\begin{tabular}{lcc}
\hline \hline \multicolumn{1}{c}{ Discovery algorithm } & RDC & Modification \\
\hline Our simple discovery algorithms & CSMA/CA & using unidirectional links \\
Our enhanced discovery algorithms & CSMA/CA & using unidirectional links \\
Our enhanced discovery algorithms & CSMA/CA & bidirectional links only \\
Collect-based & CSMA/CA & baseline (bidirectional) \\
\hline Our simple discovery algorithms & Our RDC & send phase info to node \\
Our enhanced discovery algorithms & Our RDC & send phase info to node \\
Our enhanced discovery algorithms & Our RDC & send phase info to controller \\
Our enhanced discovery algorithms & ContikiMAC & bidirectional links only \\
Collect-based & ContikiMAC & baseline (bidirectional) \\
\hline
\end{tabular}

Source: author

In addition to fully bidirectional networks, we tested three unidirectional link settings: (i) random unidirectional links (15\% of all links), which emulate unidirectional links that naturally emerge in homogeneous networks, (ii) random nodes with increased range (20\% of all nodes have double range), to represent heterogeneous networks, and (iii) a special case for SDN, in which the controller is able to reach all nodes in the network within one hop (referred to as "controller to all" in Section 6.2).

The number of nodes ranged from 16 to 100 nodes (square numbers only), to check the algorithms behavior as the network gets larger. The nodes were positioned either randomly or as square grids. The controller was positioned at a grid corner, while the data sink was placed at the grid midpoint. The positioning of these nodes was random in the random topologies. All nodes in the network transmitted constant bit rate (CBR) data, except the data sink and the controller node, which do not transmit data packets. The data payload size was 10 bytes, each node transmits 1 packet per minute, which is typical of WSN applications.

For each parameter combination, ten 60-minute-long simulation runs were executed, to achieve statistical significance. The graphs presented in the following sections show $95 \%$ confidence intervals. Every "statistically equal" or "statistically different" statement in the results description (Section 6.2) corresponds to the outcome of a two-tailed MannWhitney U-test considering $\alpha=0.05$.

A summary of the simulation parameters is presented in Table 24.

\subsubsection{Tools}

All protocols were implemented on Contiki OS version 3.0. The SDN support for wireless sensor networks in Contiki is provided by IT-SDN (ALVES et al., 2019), presented in Section 2.4.2. 
Table 24 - Simulation parameters.

\begin{tabular}{ll}
\hline \hline \multicolumn{1}{c}{ Parameter } & \multicolumn{1}{c}{ Value } \\
\hline Number of nodes & $16,25,36,49,64,81,100$ \\
\hline Topologies & random, grid \\
\hline Simulation duration & $3600 \mathrm{~s}$ \\
\hline Number of replications & 10 \\
\hline Data payload size & 10 bytes \\
\hline Data transmission rate (each node) & 1 packet $/ \mathrm{min}$ \\
\hline Node boot interval & {$[0,1] \mathrm{s}$} \\
\hline Data traffic start time & {$[2,3] \mathrm{min}$} \\
\hline ContikiMAC channel check rate $(\mathrm{CCR})$ & $8 \mathrm{~Hz}$ \\
\hline \hline
\end{tabular}

Source: author

A summary of the IT-SDN parameters we used is shown in Table 25. We configured IT-SDN to send end-to-end acknowledgements to control packets, send source-routed control packets, and limit neighbor table size to 10 entries. Only end-to-end reliability was used; although IT-SDN supports an extra hop-by-hop reliability mechanism, it was disabled as it does not work properly in unidirectional links. For the same reason, link layer acknowledgements from IEEE 802.15.4 were disabled.

Whenever using the proposed discovery algorithms, the controller software assumes that a neighbor information packet contains only the inbound neighbors of the sender; therefore, a directed edged is added in the controller representation of the network. On the other hand, when running traditional discovery protocols, the controller adds a double edge for each neighbor in the neighbor information packet: an edge from the packet sender to the neighbor, and vice-versa.

Table 25 - IT-SDN parameters.

\begin{tabular}{lll}
\hline \hline \multicolumn{1}{c}{ Parameter } & \multicolumn{1}{c}{ Value } \\
\cline { 2 - 3 } & Software version & v0.4.1 \\
\cline { 2 - 3 } & Controller retransmission timeout & $60 \mathrm{~s}$ \\
\cline { 2 - 3 } & Neighbor report maximum frequency & 1 packer per minute \\
\cline { 2 - 3 } & Neighbor table size & 10 entries \\
\cline { 2 - 2 } & Route calculation algorithm & Dijkstra \\
\cline { 2 - 2 } & Route recalculation threshold & $20 \%$ \\
\cline { 2 - 2 } & Source routed control packets & Enabled \\
\cline { 2 - 2 } & Control packets reliability & End-to-end \\
\hline
\end{tabular}

The algorithms were benchmarked with the COOJA WSN simulator/emulator tool (OSTERLIND et al., 2006), using Directed Graph Radio Medium (DGRM) to model radio links. DGRM enables defining unidirectional links, opposed to the other radio medium models available on COOJA. Each link is individually defined as an ideal communication channel, that is, packet losses at the physical layer originate from collisions only. 
We used sky/telosB mote as target compilation platform, the memory capacity of which is $10 k B$ of RAM and $48 k B$ of ROM (MEMSIC, 2011). Table 26 contains the memory usage of the compiled binaries of an SDN-enabled WSN node according to the size Linux command. Depending on the combination of algorithms, RAM usage ranges from $7080 B$ to $9538 B$, while ROM occupation goes from $35592 B$ to $44996 B$. Generally, Colletc-based discovery uses more memory than our enhanced algorithm, which in turn requires more ROM memory than the simple algorithm. Our unidirectional RDC is more memory consuming than ContikiMAC, which is expected, since it builds upon it.

Table 26 - Memory usage.

\begin{tabular}{lccc}
\hline \hline \multicolumn{1}{c}{ Discovery algorithm } & RDC & RAM usage & ROM usage \\
\hline Our simple discovery algorithms & CSMA/CA & $7116 B$ & $35592 B$ \\
Our enhanced discovery algorithms & CSMA/CA & $7080 B$ & $36378 B$ \\
Collect-based & CSMA/CA & $8964 B$ & $41554 B$ \\
\hline Our simple discovery algorithms & Our RDC & $7998 B$ & $41908 B$ \\
Our enhanced discovery algorithms & Our RDC & $7962 B$ & $42670 B$ \\
Our enhanced discovery algorithms & ContikiMAC & $7654 B$ & $39774 B$ \\
Collect-based & ContikiMAC & $9538 B$ & $44996 B$ \\
\hline \hline
\end{tabular}

Source: author

IT-SDN provides a custom controller software, which we use in our experiments with minor modifications to calculate routes with unidirectional links. It runs on the host machine and connects with the sensor network through the COOJA serial server extension. It uses the Dijkstra algorithm to calculate best routes according to link quality values provided by the neighbor discovery protocol. Routes are recalculated whenever a neighbor information packet is received, whereas new routes are deployed only if it is at least $20 \%$ better than the currently deployed route.

The size Linux command indicates the controller software text portion is 97124 bytes, while volatile memory portion is 7008 bytes; however, this does not encompass execution time memory allocation. Other controller resource usage metrics were not collected, such as energy consumption and processing, since the controller runs on the host machine and is not expected to be the bottleneck on our SDWSN architecture.

The random topologies are generated with the NPART software (MILIC; MALEK, 2009), using the default parameters for Berlin networks. The actual topologies used are available at the Scientific Data Repository from Universidade de São Paulo, at the link $<$ repositorio.uspdigital.usp.br $>$.

\subsubsection{Metrics}

We have considered the following performance metrics: 
- Data delivery: the global percentage of data packets that successfully reached their destination, calculated as $P D R=\frac{\sum_{i}(\# \text { data packets received at node } i)}{\sum_{i}(\# \text { data packets sent at node } i)}$.

- Data delay: the average time between the data packets transmission and reception (at the application layer, therefore queuing and flow setup delays are included).

- Control overhead: the total number of non-data packets transmitted within the network, which is related to the discovery algorithm's efficiency.

- Energy consumption: the average amount of energy spent by the radio transceiver of all network nodes, considering three radio states (transmitting, receiving, and sleeping). We have used Energest (DUNKELS et al., 2007), a tool from Contiki OS, to obtain the amount of time spent in each state. The energy consumption is calculated as $E=V\left(I_{t} T_{t}+I_{r} T_{r}+I_{s} T_{s}\right)$, where $V, T$, and $I$ are, respectively, the voltage, time spent in each state, and current drawn in each stage; the subscripts $t, r$ and $s$ refer to transmitting, receiving and sleeping states, respectively. Drained current values have been taken from the CC2420 datasheet (TEXAS INSTRUMENTS, 2004) and replicated in Table 27.

- Link discovery rate: the percentage of existing links that the neighbor discovery algorithm was able to detect throughout the simulation. The discovery rate is measured at the controller, considering its internal representation of the network.

Table 27 - Energy parameters.

\begin{tabular}{ll}
\hline \hline \multicolumn{1}{c}{ Parameter } & \multicolumn{1}{c}{ Value } \\
\hline Radio module transmission power & $0 \mathrm{dBm}$ \\
\hline Transmission current consumption & $21.70 \mathrm{~mA}$ \\
\hline Receiving current consumption & $22.00 \mathrm{~mA}$ \\
\hline Sleeping current consumption & $0.18 \mathrm{~mA}$ \\
\hline Operation voltage & $3 \mathrm{~V}$ \\
\hline \hline
\end{tabular}

Source: Texas Instruments (2004)

\subsection{Results}

The results are organized as follows: each metric is presented as a set of four graphs, one for each link type (i.e. bidirectional links only, controller to all, nodes with increased range, and random unidirectional links). Within the graphs, each line represents a combination of three simulation parameters: (i) neighbor discovery algorithm (i.e. collect or this work); (ii) radio duty cycling protocol (i.e. pure CSMA, ContikiMAC, or this work); and (iii) topology type (i.e. random or grid). All numeric metric values and confidence intervals are available at the Scientific Data Repository from Universidade de São Paulo 
(<repositorio.uspdigital.usp.br $>$ ). The non-RDC, pure CSMA results are discussed in Section 6.2.1, while the experiments with RDC are discussed in Section 6.2.2.

\subsubsection{Simulation results without duty cycling}

This section contains experiment results for non-duty-cycled pure CSMA networks, performed with the objective of assessing the impact of using unidirectional links for routing without the influence of radio duty cycling.

Figures 18, 19, 20, and 21 display data delivery results. In fully bidirectional networks, the enhanced discovery algorithms perform as good as the Collect-based approach, presenting statistically equivalent results in most network sizes (Figure 18). However, the enhanced ND/CD performs worse if unidirectional links are blacklisted (between $5 \%$ and $30 \%$ worse), although this is only statistically significant in the grid topology. The reason behind these results is that each direction of the link is informed separately, creating temporary unidirectional links in the controller representation that cannot be used for routing.

Figure 18 - Pure CSMA. Metric: Data delivery. Link setting: Bidirectional links only.

$\begin{array}{ll}- \text { Collect ND/CD (random topology) } & -\$-\text { Collect ND/CD (grid topology) } \\ - \text { Enhanced ND/CD -- bidirectional routes only (random topology) } & - \text { - Enhanced ND/CD -- bidirectional routes only (grid topology) } \\ - \text { Enhanced ND/CD (random topology) } & -4-\text { Enhanced ND/CD (grid topology) } \\ - \text { Naive ND/CD (random topology) } & =- \text { Naive ND/CD (grid topology) }\end{array}$

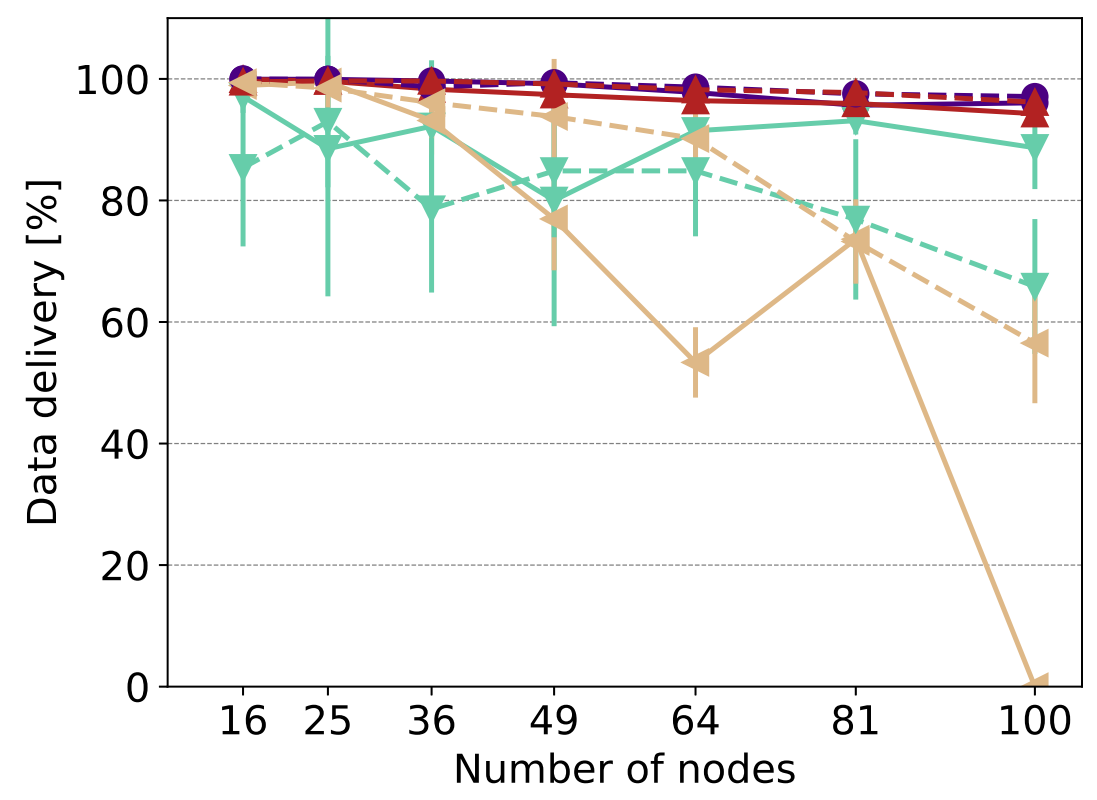

Source: author

In fact, considering our enhanced algorithm, we can observe an increased packet delivery rate when using unidirectional links in the other link scenarios (Figures 19, 20, and 21). Enabling the use of unidirectional links yields at least $90 \%$ delivery, while not using these links can drop the delivery rate into the realm of $80 \%$. We can notice a larger impact 
Figure 19 - Pure CSMA. Metric: Data delivery. Link setting: Controller to all.
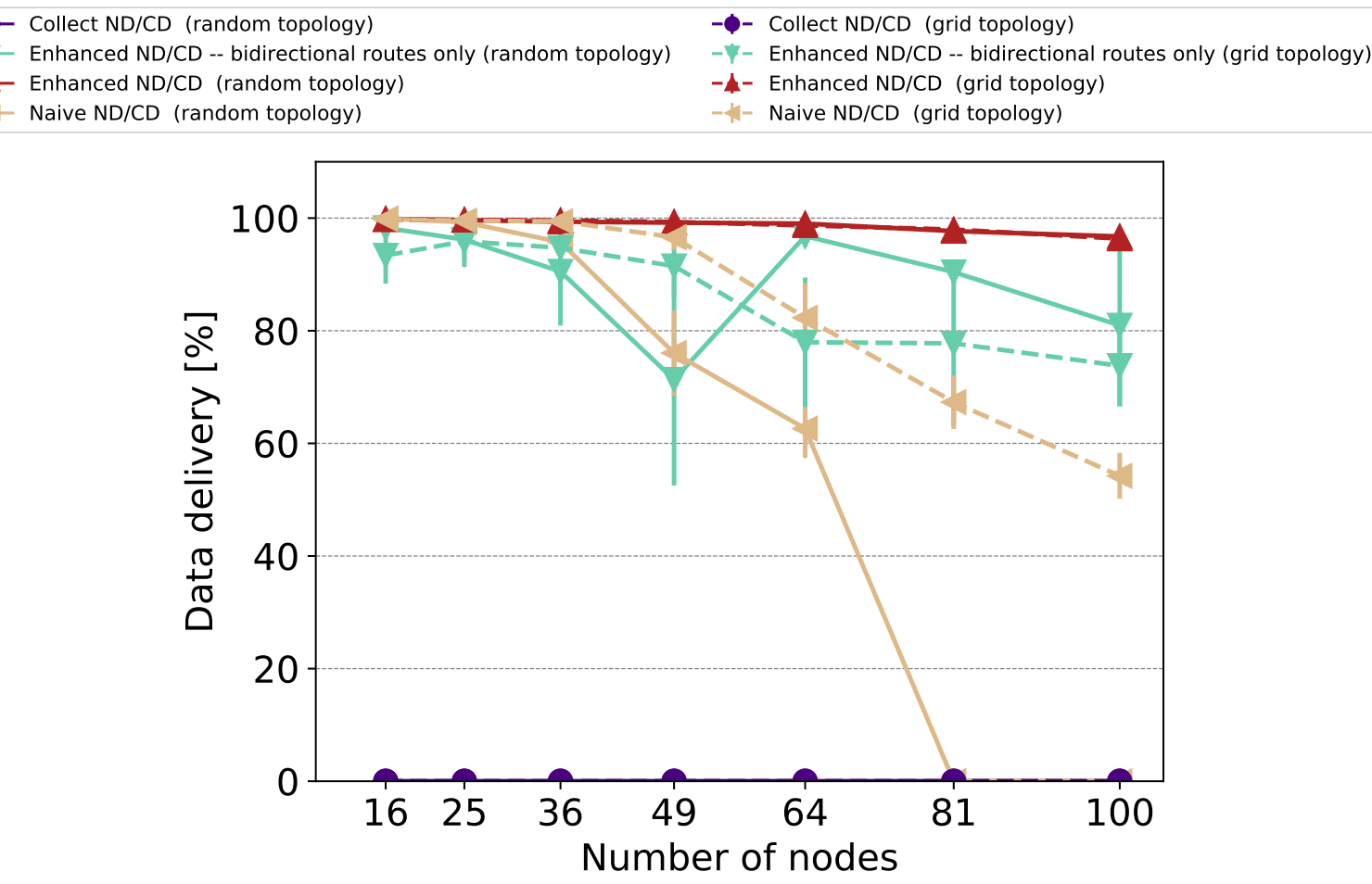

Source: author

Figure 20 - Pure CSMA. Metric: Data delivery. Link setting: Nodes with increased range.

\begin{tabular}{|ll}
- Collect ND/CD (random topology) & - - Collect ND/CD (grid topology) \\
- Enhanced ND/CD -- bidirectional routes only (random topology) & - - Enhanced ND/CD -- bidirectional routes only (grid topology) \\
- Enhanced ND/CD (random topology) & $-4-$ Enhanced ND/CD (grid topology) \\
- - Naive ND/CD (random topology) & - - Naive ND/CD (grid topology)
\end{tabular}

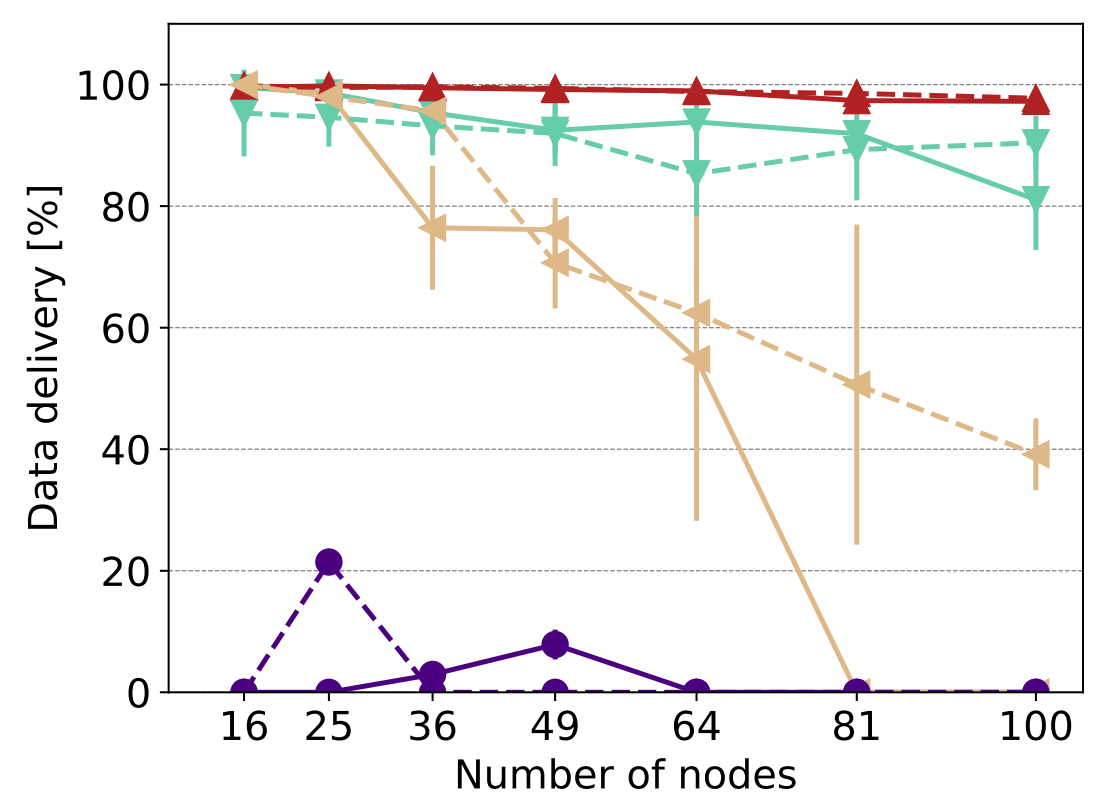

Source: author 
Figure 21 - Pure CSMA. Metric: Data delivery. Link Setting: Random unidirectional links.

\begin{tabular}{|ll}
- - Collect ND/CD (random topology) & - - Collect ND/CD (grid topology) \\
- Enhanced ND/CD -- bidirectional routes only (random topology) & - - Enhanced ND/CD -- bidirectional routes only (grid topology) \\
- Enhanced ND/CD (random topology) & $-4-$ Enhanced ND/CD (grid topology) \\
- - Naive ND/CD (random topology) & - - Naive ND/CD (grid topology)
\end{tabular}

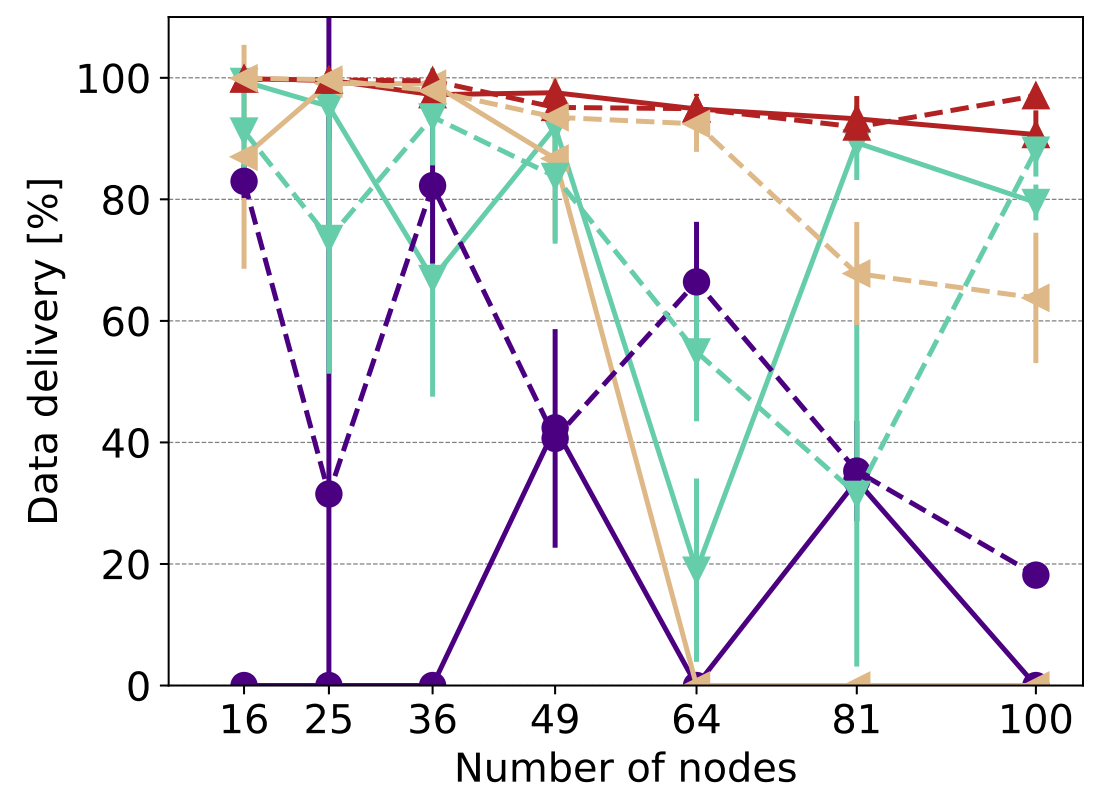

Source: author

in the "controller to all" and "random unidirectional links" topologies. The delivery rate difference between using and not using unidirectional links is statistically significant for all scenarios with unidirectional links, except for 16-node and 81-node random topologies with random unidirectional links.

It is notable that some curves do not present a monotonic delivery decrease as the number of nodes increases, especially in Figure 21. The reason behind that is the randomness in topology and unidirectional link placement. Particularly, these two factors may be combined in such a way that the remaining bidirectional links become bottlenecks. As such, as the results show, using unidirectional links increases robustness.

The simple ND/CD algorithms presented comparable performance to the enhanced algorithms in small networks (up to 36 nodes). However, its lack of scalability becomes evident, as the delivery rate decreases in a linear trend as the network size increases. In large random topologies, it is unable to deliver packets at all; while it performs better on grid topologies, delivering between $39 \%$ and $64 \%$ on 100 -node grids.

The Collect-based algorithm performs poorly in the presence of unidirectional links, regardless of link and topology type, presenting statistically different data delivery results from the enhanced solution. 
Figure 22 - Pure CSMA. Metric: Data delay. Link setting: Bidirectional links only.
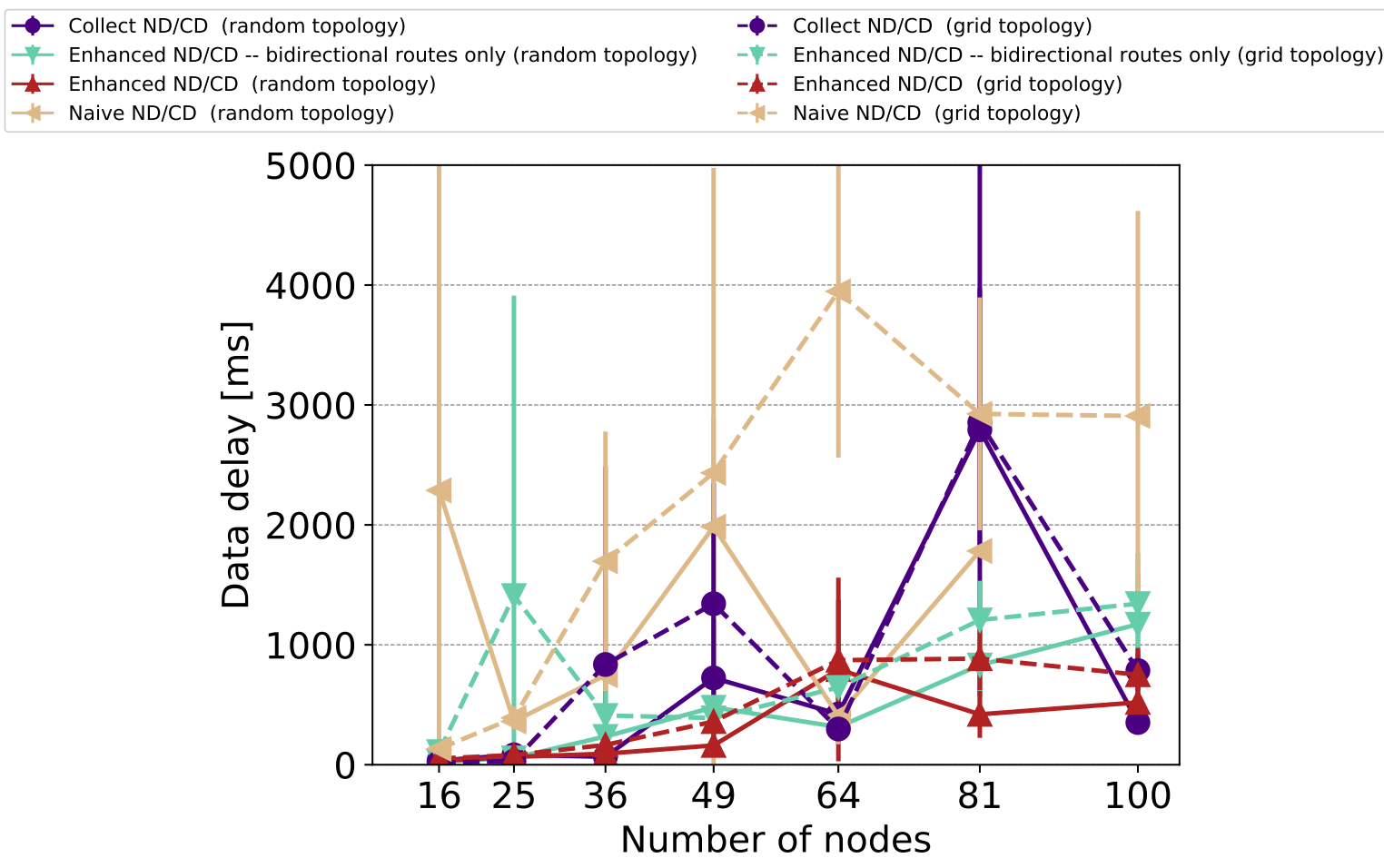

Source: author

Figure 23 - Pure CSMA. Metric: Data delay. Link setting: Controller to all.

$\begin{array}{ll}- \text { - Collect ND/CD (random topology) } & -\$-\text { Collect ND/CD (grid topology) } \\ - \text { Enhanced ND/CD -- bidirectional routes only (random topology) } & - \text { - Enhanced ND/CD -- bidirectional routes only (grid topology) } \\ - \text { Enhanced ND/CD (random topology) } & -4-\text { Enhanced ND/CD (grid topology) } \\ - \text { - Naive ND/CD (random topology) } & - \text { - Naive ND/CD (grid topology) }\end{array}$

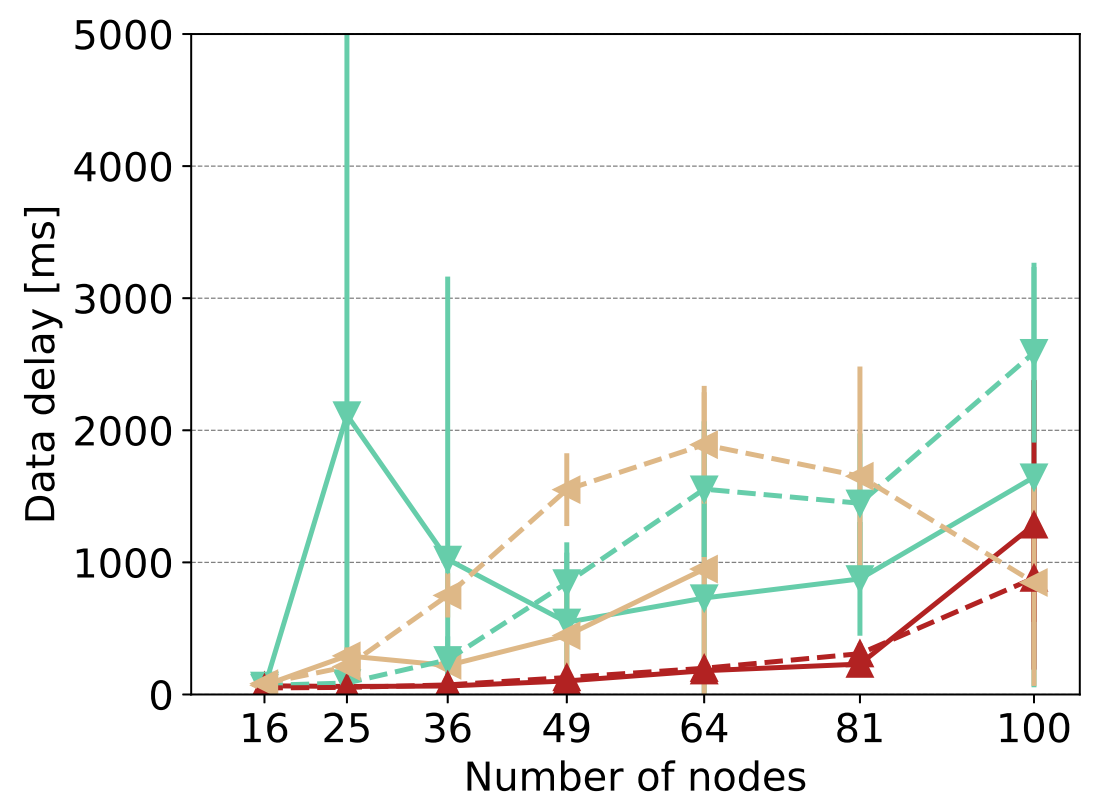

Source: author 
Figure 24 - Pure CSMA. Metric: Data delay. Link setting: Nodes with increased range.
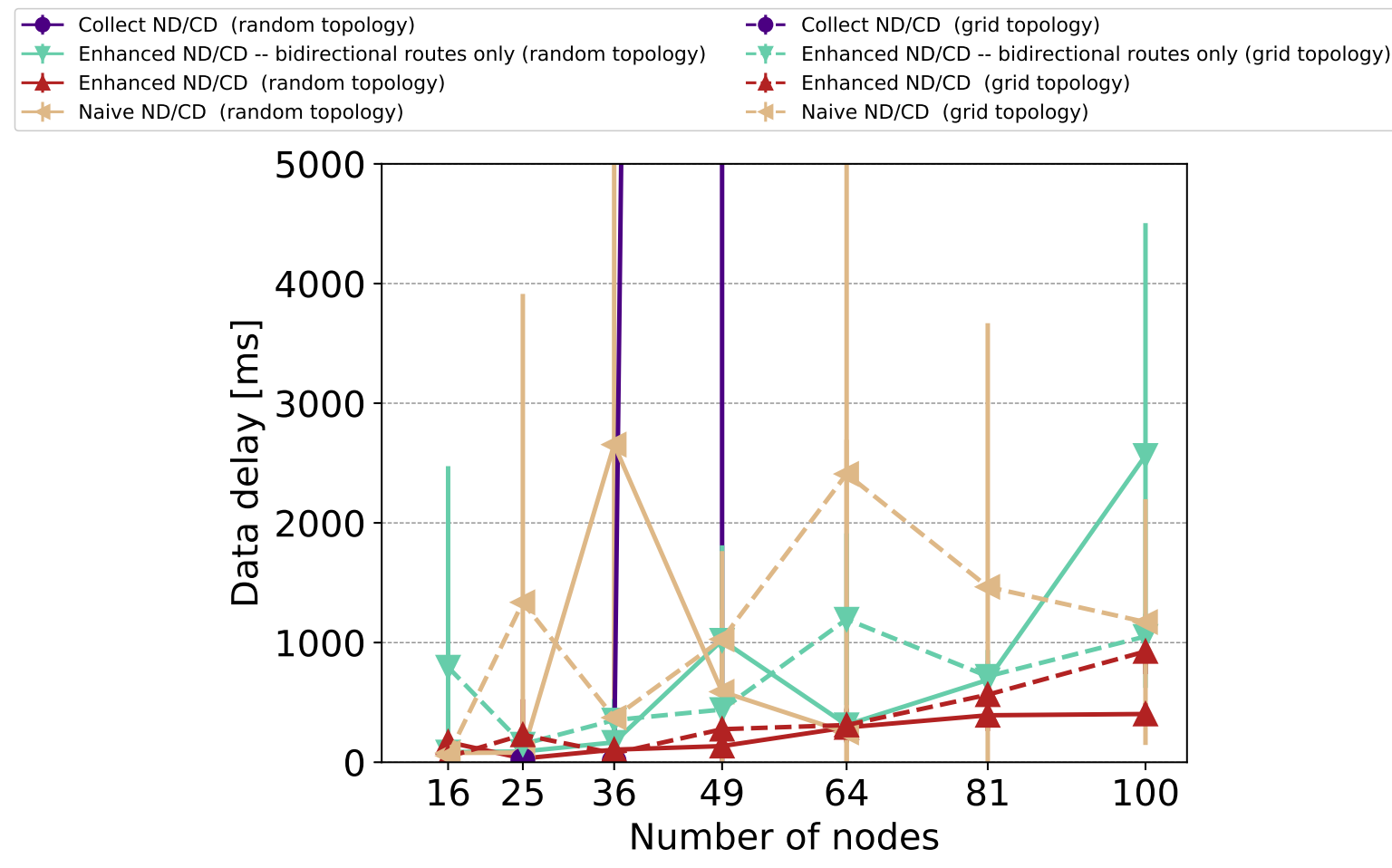

Source: author

Figure 25 - Pure CSMA. Metric: Data delay. Link setting: Random unidirectional links.

\begin{tabular}{|ll}
- - Collect ND/CD (random topology) & $-\$-$ Collect ND/CD (grid topology) \\
- Enhanced ND/CD -- bidirectional routes only (random topology) & - - Enhanced ND/CD -- bidirectional routes only (grid topology) \\
- Enhanced ND/CD (random topology) & $-4-$ Enhanced ND/CD (grid topology) \\
- - Naive ND/CD (random topology) & - - Naive ND/CD (grid topology)
\end{tabular}

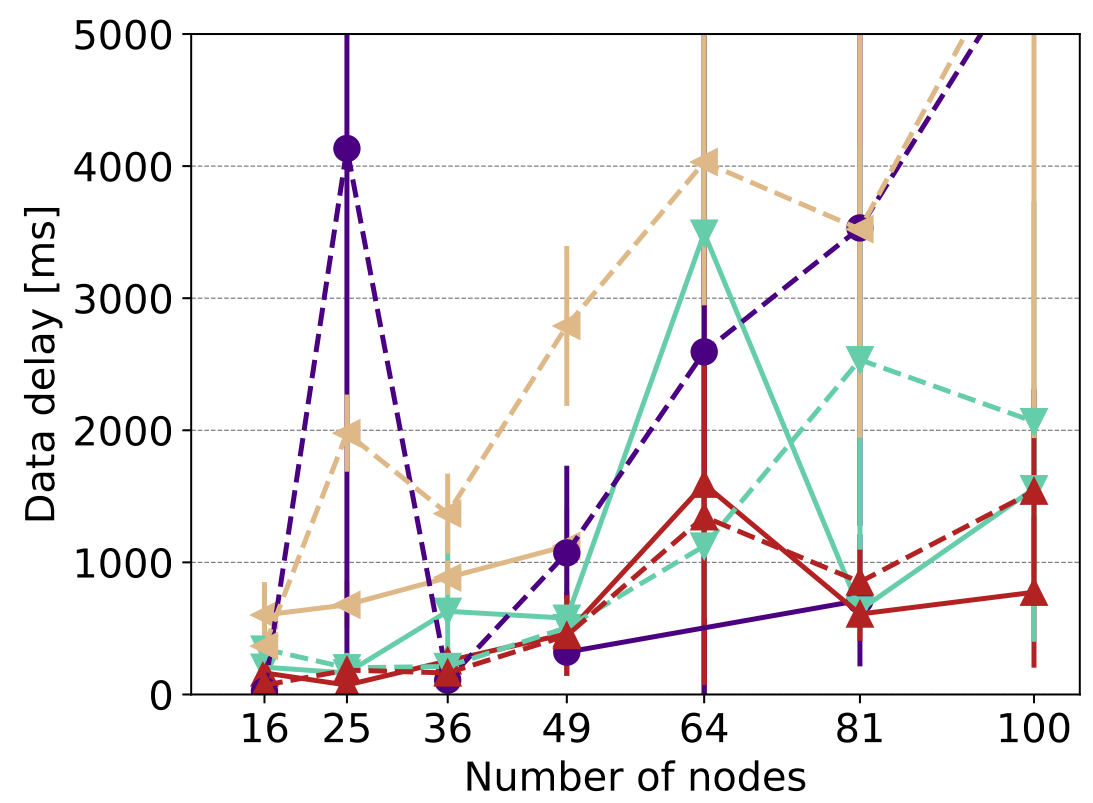

Source: author 
Considering the enhanced ND/CD results, the "controller to all" topology provides the largest delay improvement when unidirectional links are employed, in comparison to the case only bidirectional links are allowed (Figure 23). The improvement is at least $30 \%$ on large topologies, presenting statistically different results for most topology sizes (exceptions are 16-node and 100-node random topologies, and the 25-node grid). The unidirectional links from the controller enable a fast flow setup on all nodes. Conversely, the presence of unidirectional links increases link layer contention, even if such links are not used.

For the other link setting scenarios, still considering the enhanced ND/CD algorithm, the unidirectional links tend to decrease the average delay and standard deviation. Nonetheless, the standard deviation intervals overlap in many cases, which hinders the drawing of further conclusions.

An investigation of simulation logs revealed that the causes of high confidence intervals in the delay metric are at least one of the following three reasons: (i) the delay metric is sensitive to the initial network transient; (ii) some of the 10 simulation runs for a given scenario did not deliver any data packets, therefore, it was not possible to calculate delay for that particular run. Consequently, the average delay was calculated with less than 10 data points, which tends to raise the confidence interval; (iii) one of the simulation runs yielded a significantly larger delay than the other ones (at least triple the average). This may happen because, within a specific simulation, a couple nodes were able to deliver data only near the end of the simulation time. As a consequence, the delivery of data packets that were buffered since the beginning of the simulation cause the average packet delay for those nodes to be in the order of magnitude of tens of minutes, much larger the usual steady state delay (around 100ms). Notice that our improved solution supporting unidirectional links presents the narrowest confidence interval in most cases, which indicates this is the most consistent algorithm, that is, it is less susceptible to the aforementioned issues.

The Collect ND/CD fails to deliver any data packet in many most scenarios with unidirectional links, causing the absence of data points in Figures 23, 24 and 25. In these scenarios, a data packet is delivered only after a long queuing delay caused by the long time it takes to first configure the flow tables. With all links bidirectional, in Figure 22, sometimes the Collect ND/CD performs better, sometimes it performs worse than the enhanced ND/CD algorithm, without a clear pattern. We noticed, however, that whenever an algorithm presented higher delay, it was accompanied by a larger confidence interval.

The simple ND/CD showed the higher delay among all algorithms, as observed in Figures 22, 23, 24, and 25. This is a consequence of longer initial delay and convergence time. On some cases, the simple ND/CD presented a declining delay trend on large topologies (clearly in Figure 23), but that occurs because nodes farther from the controller 
never get their flow tables configured, giving a false illusion of better delay performance.

From Figures 26, 27, 28, and 29, the enhanced ND/CD discovered nearly all links in all topologies (at least $95 \%$ on $65 \%$ of the test cases, minimum $89 \%$ ), while the collect-based discovery failed to find most of the unidirectional links, and the naive algorithm presented scalability issues, consistently presenting discovery above $80 \%$ only on networks up to 36 nodes.

Figure 26 - Pure CSMA. Metric: Link discovery rate. Link setting: Bidirectional links only.
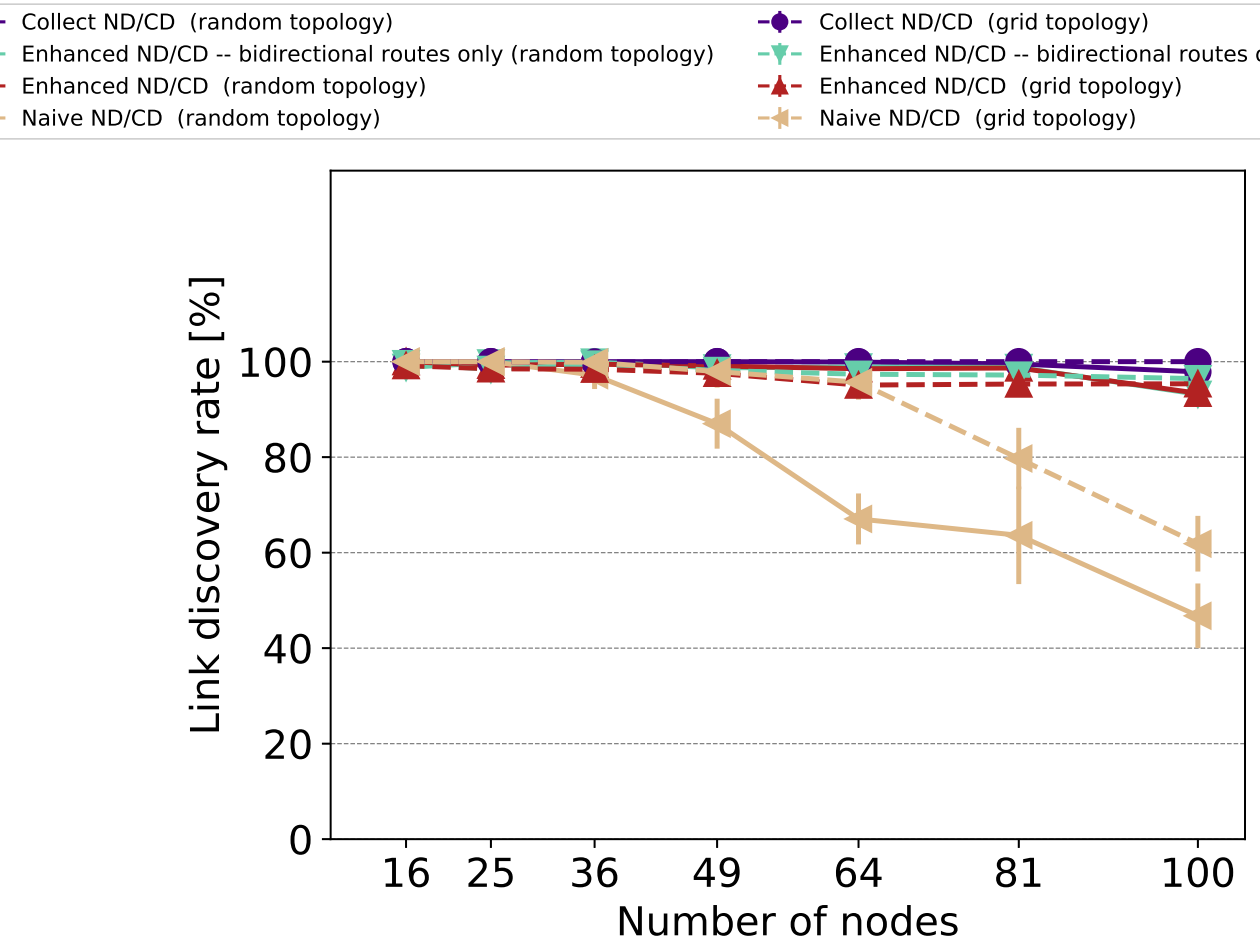

Source: author

A neighborhood larger than the neighbor table capacity causes our algorithm to discover less than $100 \%$ of the links, as the exceeding links are ignored. The other reason for undetected links is the lack of a bidirectionally connected component, as the nodes without a bidirectional path towards the controller are unable to send neighbor report packets (e.g., 49-node and 81-node random unidirectional links scenarios, Figure 29).

The naive algorithm performed worse on random than grid topologies, as the number of links per node can be large, quickly depleting the limited amount of memory reserved to store reachability information. From 64-node scenarios onward, the number of links is just too large, hampering whole network discovery.

Collect-based discovery performed as good as our algorithm for fully bidirectional networks, the difference is that it stores the outbound neighbors while we store the inbound neighbors, causing small differences. On the other hand, collect failed to detect unidirectional links, degrading the observed performance on the other test cases. 
Figure 27 - Pure CSMA. Metric: Link discovery rate. Link setting: Controller to all.

$\begin{array}{ll}- \text { - Collect ND/CD (random topology) } & -\$-\text { Collect ND/CD (grid topology) } \\ - \text { Enhanced ND/CD -- bidirectional routes only (random topology) } & - \text { - Enhanced ND/CD -- bidirectional routes only (grid topology) } \\ - \text { Enhanced ND/CD (random topology) } & -4-\text { Enhanced ND/CD (grid topology) } \\ - \text { - Naive ND/CD (random topology) } & - \text { - Naive ND/CD (grid topology) }\end{array}$

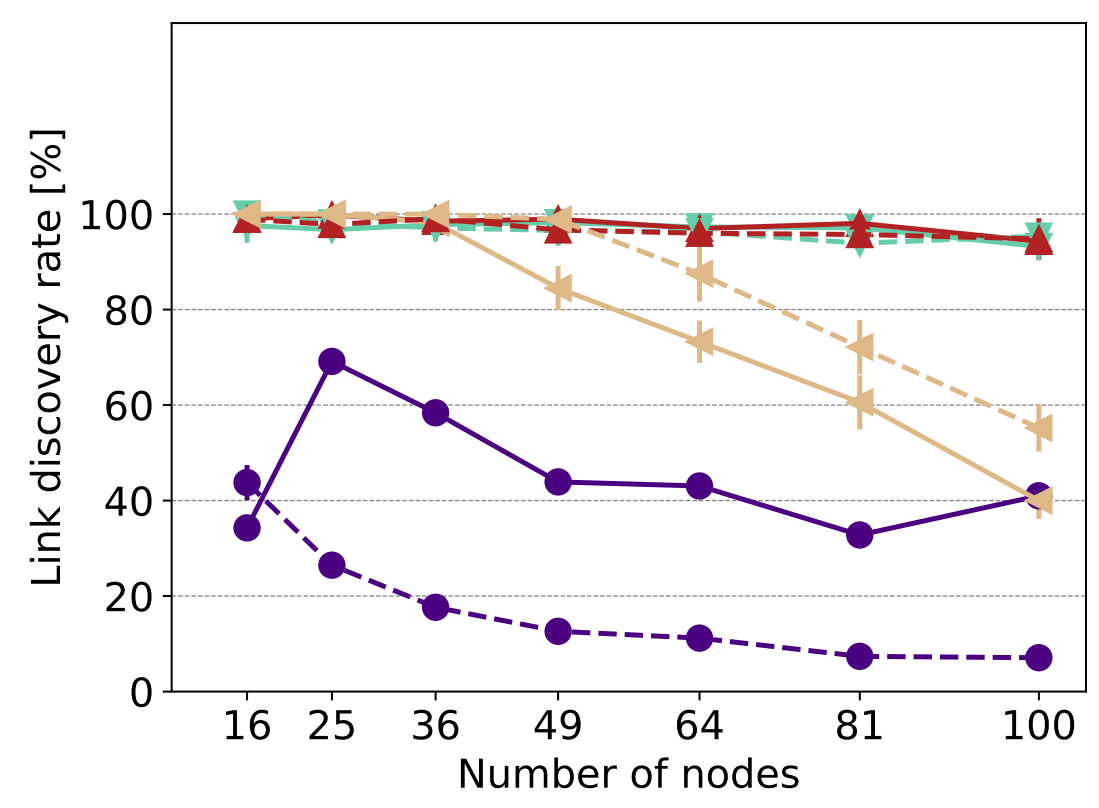

Source: author

Figure 28 - Pure CSMA. Metric: Link discovery rate. Link setting: Nodes with increased range.

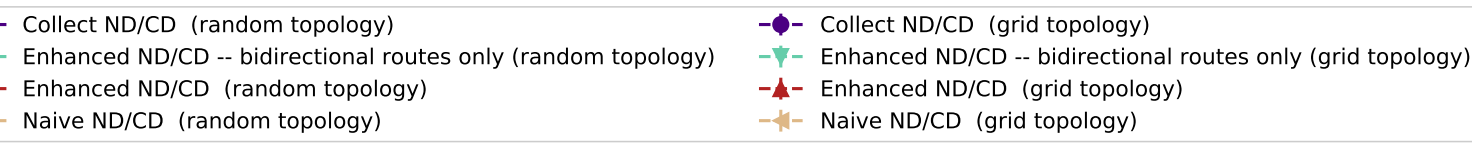

- Collect ND/CD (grid topology)

- Enhanced ND/CD -- bidirectional routes only (grid topology)

-4- Enhanced ND/CD (grid topology)

-4 - Naive ND/CD (grid topology)

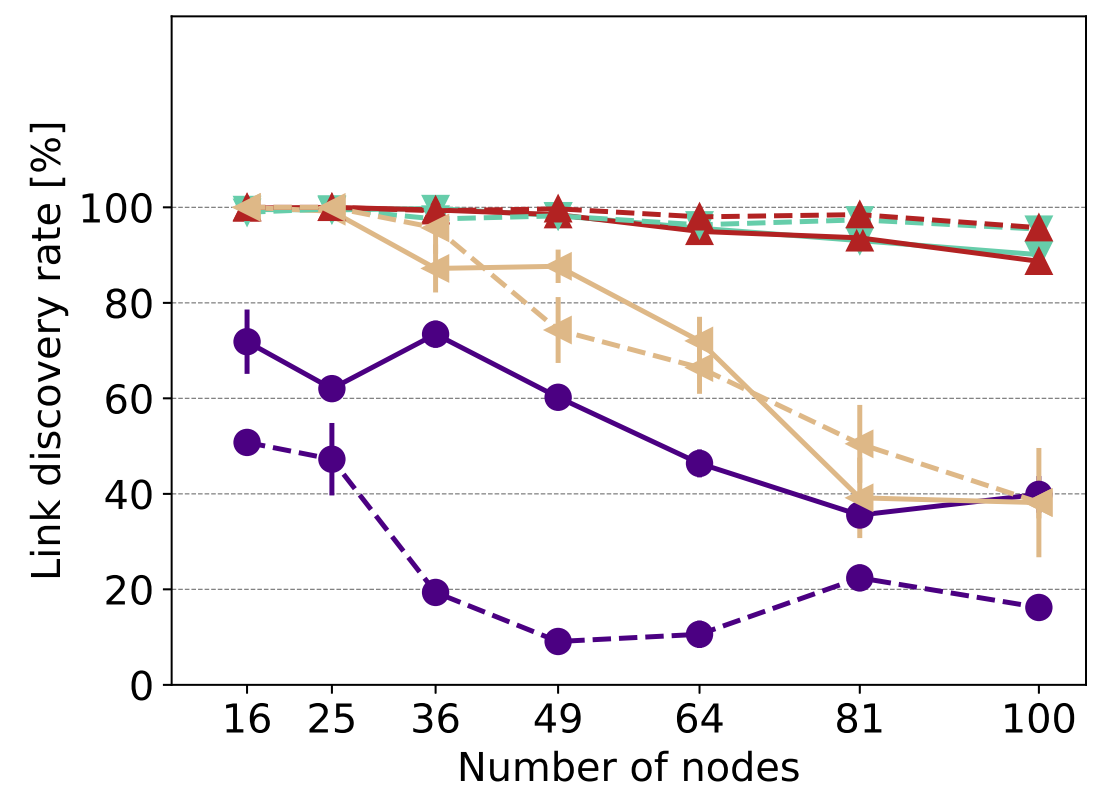

Source: author 
Figure 29 - Pure CSMA. Metric: Link discovery rate. Link setting: Random unidirectional links.

\begin{tabular}{|ll}
- - Collect ND/CD (random topology) & - - Collect ND/CD (grid topology) \\
- Enhanced ND/CD -- bidirectional routes only (random topology) & - - Enhanced ND/CD -- bidirectional routes only (grid topology) \\
- Enhanced ND/CD (random topology) & $-4-$ Enhanced ND/CD (grid topology) \\
- - Naive ND/CD (random topology) & - - Naive ND/CD (grid topology)
\end{tabular}

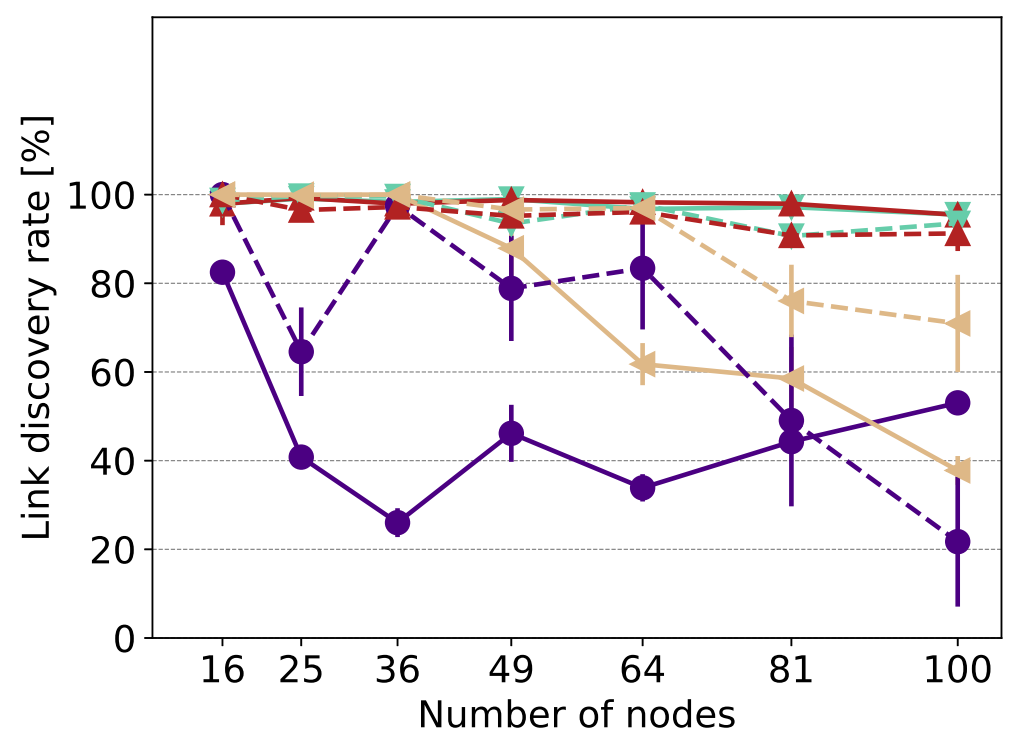

Source: author

Lastly, let us investigate the control overhead generated by each discovery algorithm, exhibited in Figures 30, 31, 32, and 33. Each tested algorithm presented linearly increasing overhead as the network size grows, although presenting different linear coefficients. The enhanced algorithm presented the most consistent results across the different link configurations. Its results with unidirectional links enabled also presented the lowest overhead whenever there is unidirectional links in the topology, although Collect wastes around $22 \%$ less on average at the fully bidirectional network.

The number of control packets shown in the graphs accounts for every kind of control packet, including discovery beacons, neighbor reports to the controller, flow requests and flow setups. Analyzing the distribution of the control packet types gives further insight into the behavior of each algorithm.

With respect to discovery beacons, the enhanced algorithm transmits at least $68 \%$ less packets than collect, $92 \%$ on average. This is the effect of the beacon reducing mechanisms implemented in our approach (overhearing and dynamic beacon intervals).

On the other hand, the number of neighbor report packets the nodes send to the controller is larger in the enhanced algorithm, notably at controller-to-all link type. This indicates that our link quality estimator is more sensitive than the one used by the Collect $\mathrm{ND} / \mathrm{CD}$, thus, there is room for fine-tuning our estimator parameters. For example, the controller transmits a lot of packets, causing the calculated LQE to vary in controller-to-all link scenarios. 
Figure 30 - Pure CSMA. Metric: Control overhead. Link setting: Bidirectional links only.

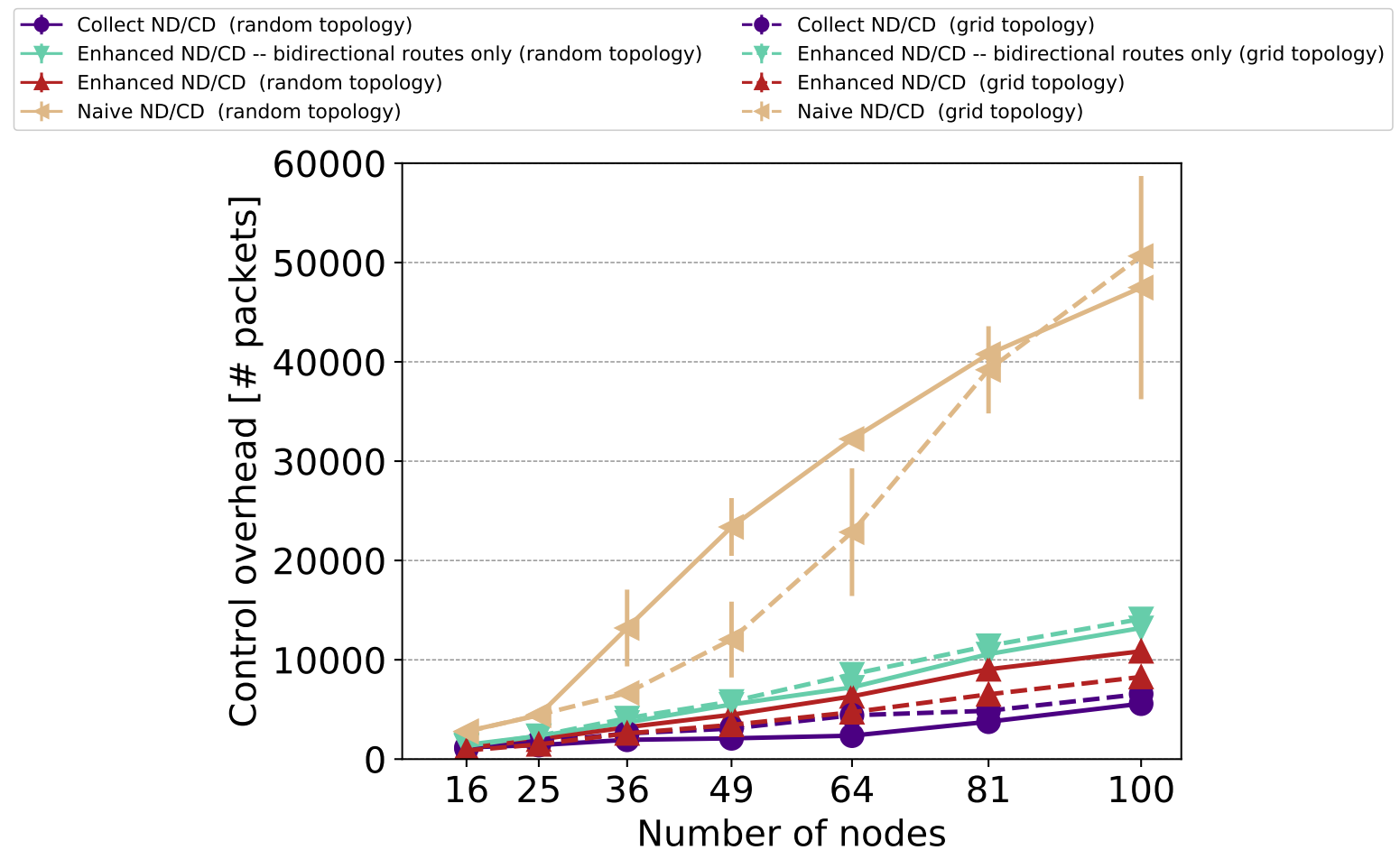

Source: author

Figure 31 - Pure CSMA. Metric: Control overhead. Link setting: Controller to all.

$\begin{array}{ll}- \text { - Collect ND/CD (random topology) } & -\$-\text { Collect ND/CD (grid topology) } \\ - \text { Enhanced ND/CD -- bidirectional routes only (random topology) } & - \text { - Enhanced ND/CD -- bidirectional routes only (grid topology) } \\ - \text { Enhanced ND/CD (random topology) } & -4-\text { Enhanced ND/CD (grid topology) } \\ - \text { - Naive ND/CD (random topology) } & - \text { - Naive ND/CD (grid topology) }\end{array}$

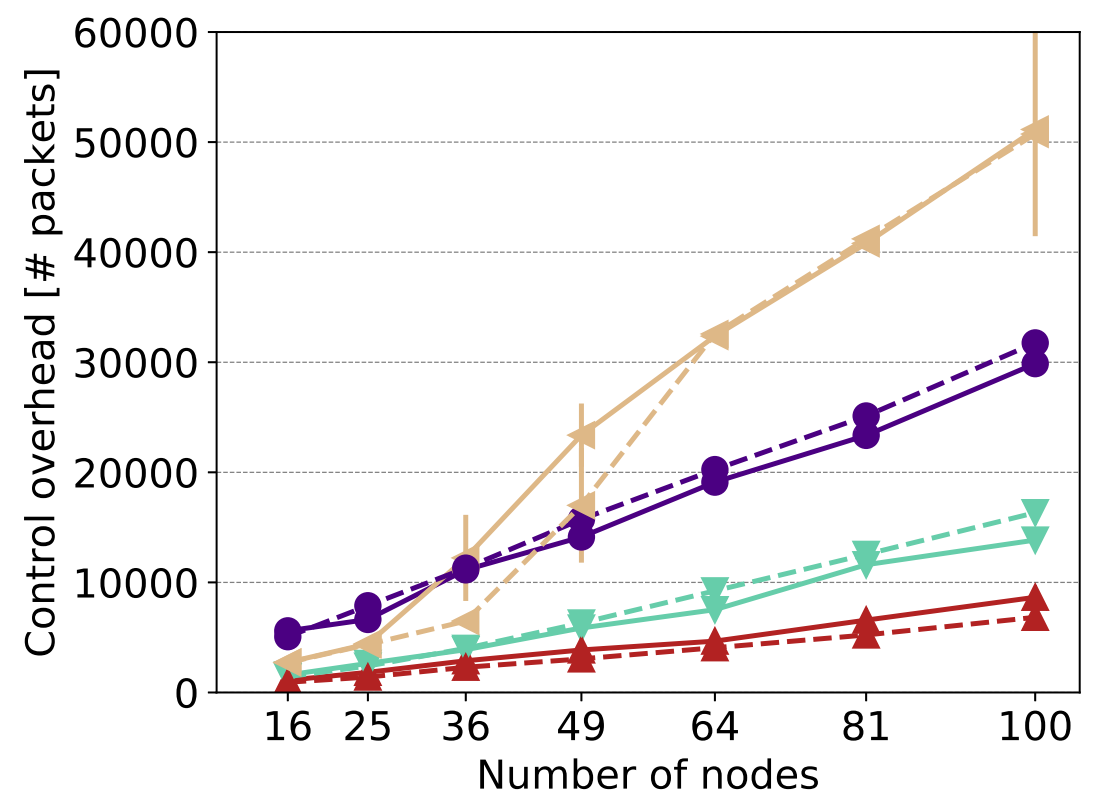

Source: author 
Figure 32 - Pure CSMA. Metric: Control overhead. Link setting: Nodes with increased range.

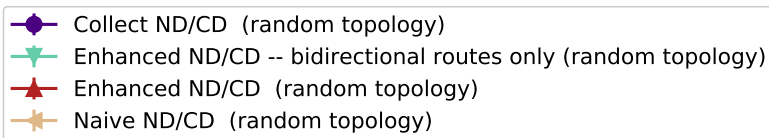

- Enhanced ND/CD -- bidirectional routes only (random topology)

t- Enhanced ND/CD (random topology)

Naive ND/CD (random topology)

- - Collect ND/CD (grid topology)

- Enhanced ND/CD -- bidirectional routes only (grid topology)

- - Enhanced ND/CD (grid topology)

$-\$-$ Naive ND/CD (grid topology)

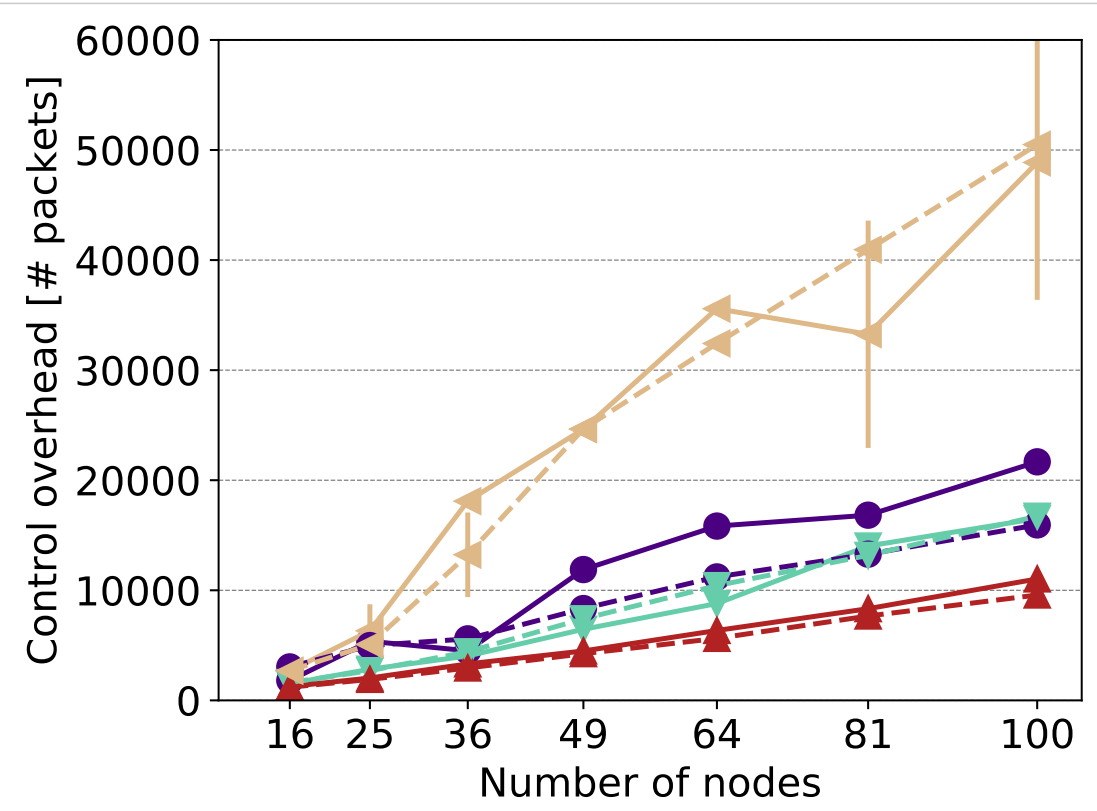

Source: author

Figure 33 - Pure CSMA. Metric: Control overhead. Link setting: Random unidirectional links.

$\begin{array}{ll}- \text { - Collect ND/CD (random topology) } & -\$-\text { Collect ND/CD (grid topology) } \\ - \text { Enhanced ND/CD -- bidirectional routes only (random topology) } & - \text { - Enhanced ND/CD -- bidirectional routes only (grid topology) } \\ - \text { Enhanced ND/CD (random topology) } & -4-\text { Enhanced ND/CD (grid topology) } \\ - \text { - Naive ND/CD (random topology) } & - \text { - Naive ND/CD (grid topology) }\end{array}$

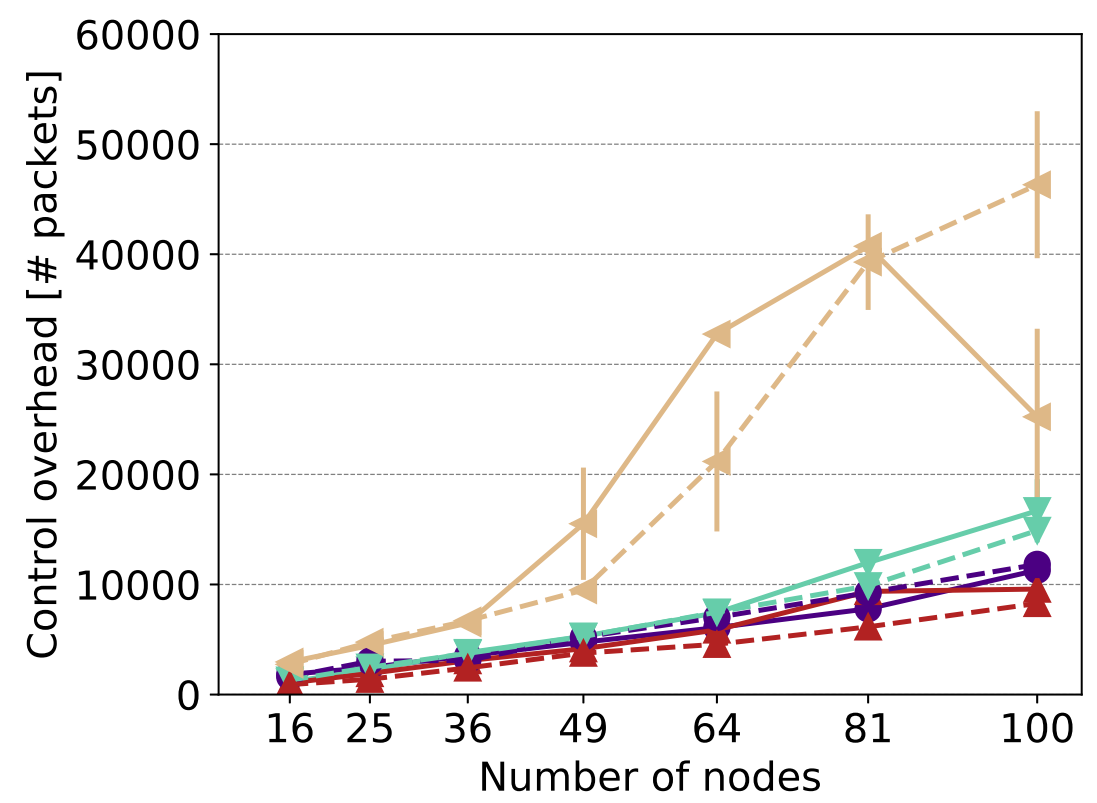

Source: author 
More neighbor report packets reaching the controller may lead to route recalculation, thus, triggering transmission of flow setup packets. We notice a correlation between the transmission frequency of these packet types. On the one hand, the naive algorithm does not estimate the link quality, therefore, it transmits very few neighbor report and flow setup packets. On the other hand, it is not equipped with any mechanism to curb the overhead of the discovery packets, which is its major source of control overhead.

We do not show energy consumption results for the no-RDC experiments, because, in these case, the radio does not enter the sleep state. Since the current drawn by transmitting and sending packets are nearly equal, the average energy consumption is approximately constant.

The conclusion drawn from these results is that leveraging unidirectional links, when they are present in the network, positively affects packet delivery rate and tends to decrease the average data delay.

\subsubsection{Simulation results with duty cycling}

This section presents and discusses the results of the discovery algorithms combined with RDCs. As observed in Section 6.2.1, the usage of unidirectional links revealed a positive impact on network performance indicators. In this section we explore how the introduction of an RDC influences the metrics.

Figure 34 - Duty cycled. Metric: Data delivery. Link setting: Bidirectional links only.
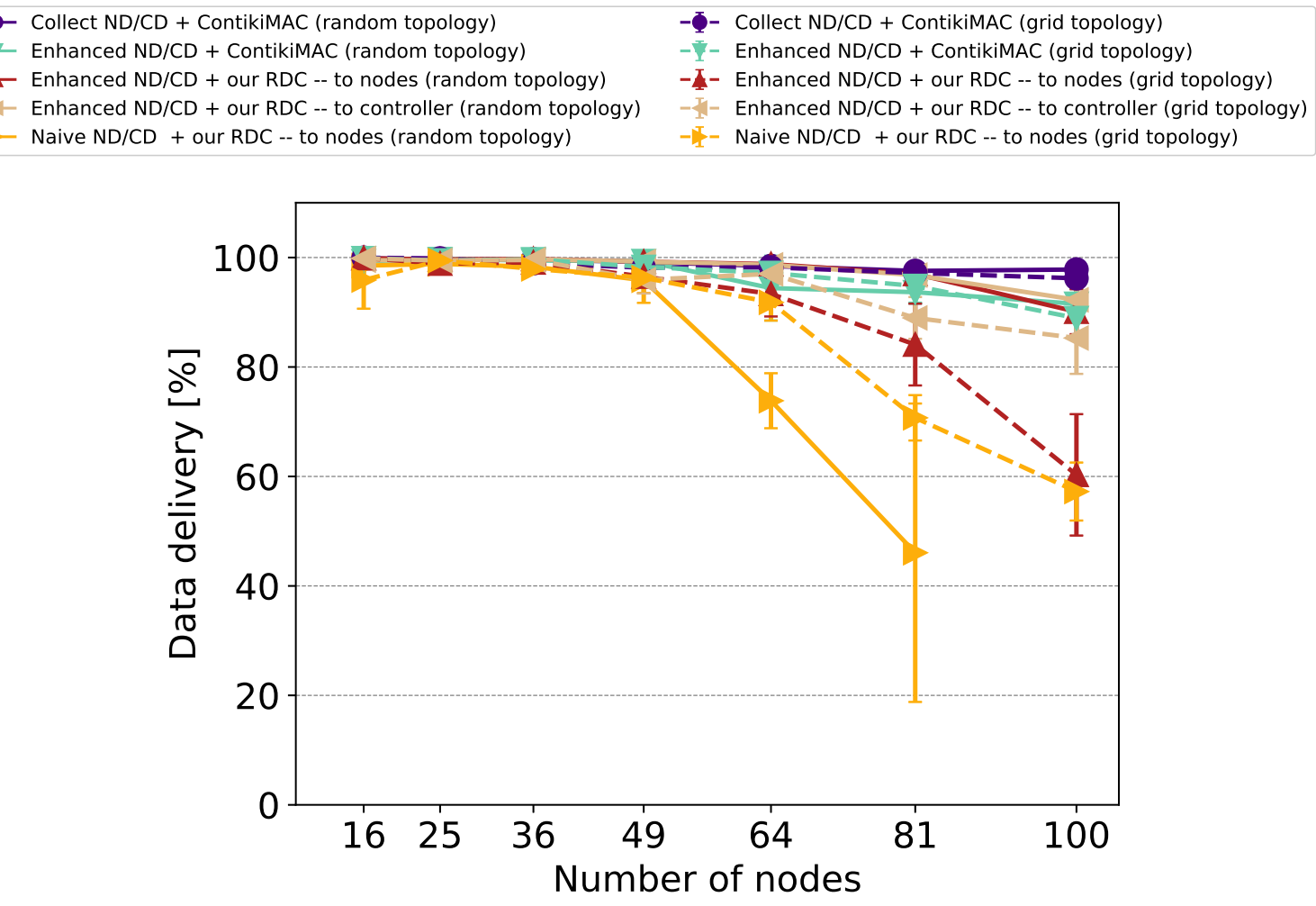

Source: author 
Figure 35 - Duty cycled. Metric: Data delivery. Link setting: Controller to all.

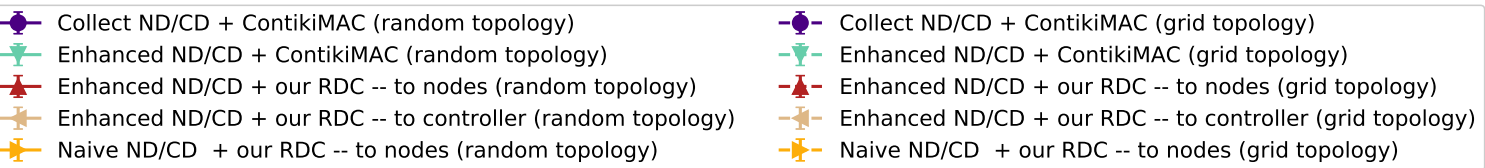

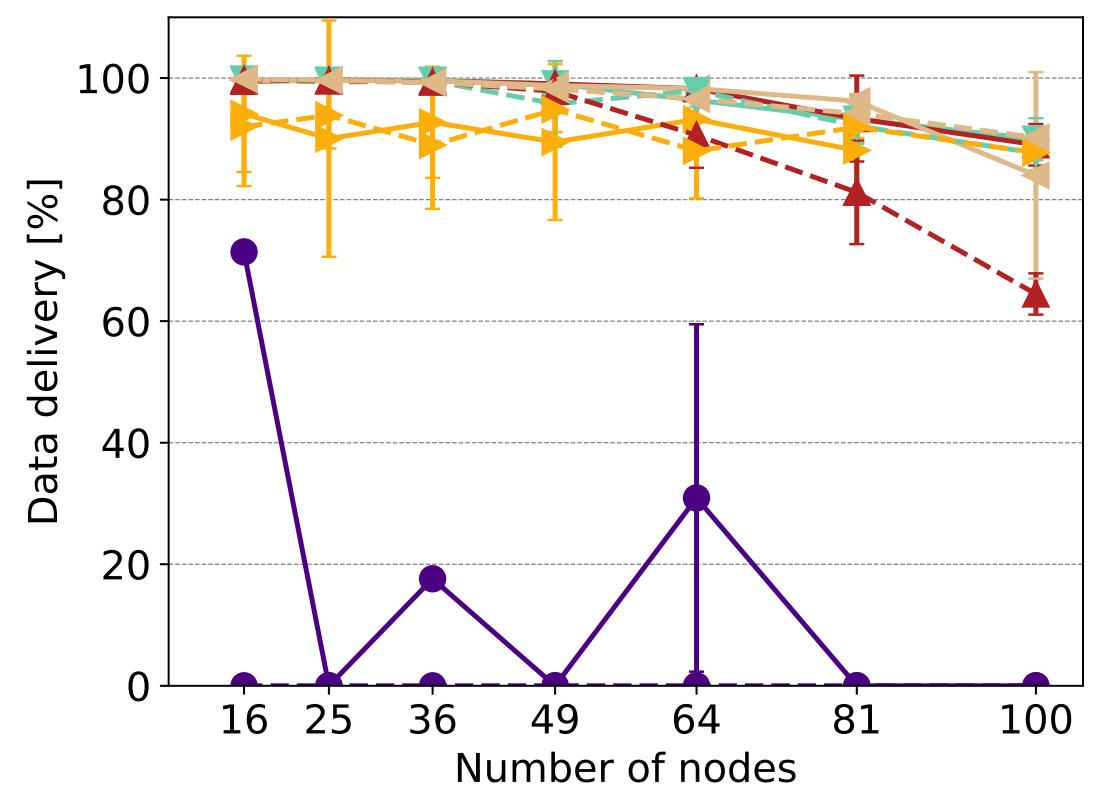

Source: author

Figure 36 - Duty cycled. Metric: Data delivery. Link setting: Nodes with increased range.

\begin{tabular}{ll} 
- Collect ND/CD + ContikiMAC (random topology) & $-\mathbf{-}$ - Collect ND/CD + ContikiMAC (grid topology) \\
- Enhanced ND/CD + ContikiMAC (random topology) & - - Enhanced ND/CD + ContikiMAC (grid topology) \\
- Enhanced ND/CD + our RDC -- to nodes (random topology) & - - Enhanced ND/CD + our RDC -- to nodes (grid topology) \\
- Enhanced ND/CD + our RDC -- to controller (random topology) & - - Enhanced ND/CD + our RDC -- to controller (grid topology) \\
- Naive ND/CD + our RDC -- to nodes (random topology) & - - Naive ND/CD + our RDC -- to nodes (grid topology) \\
\hline
\end{tabular}

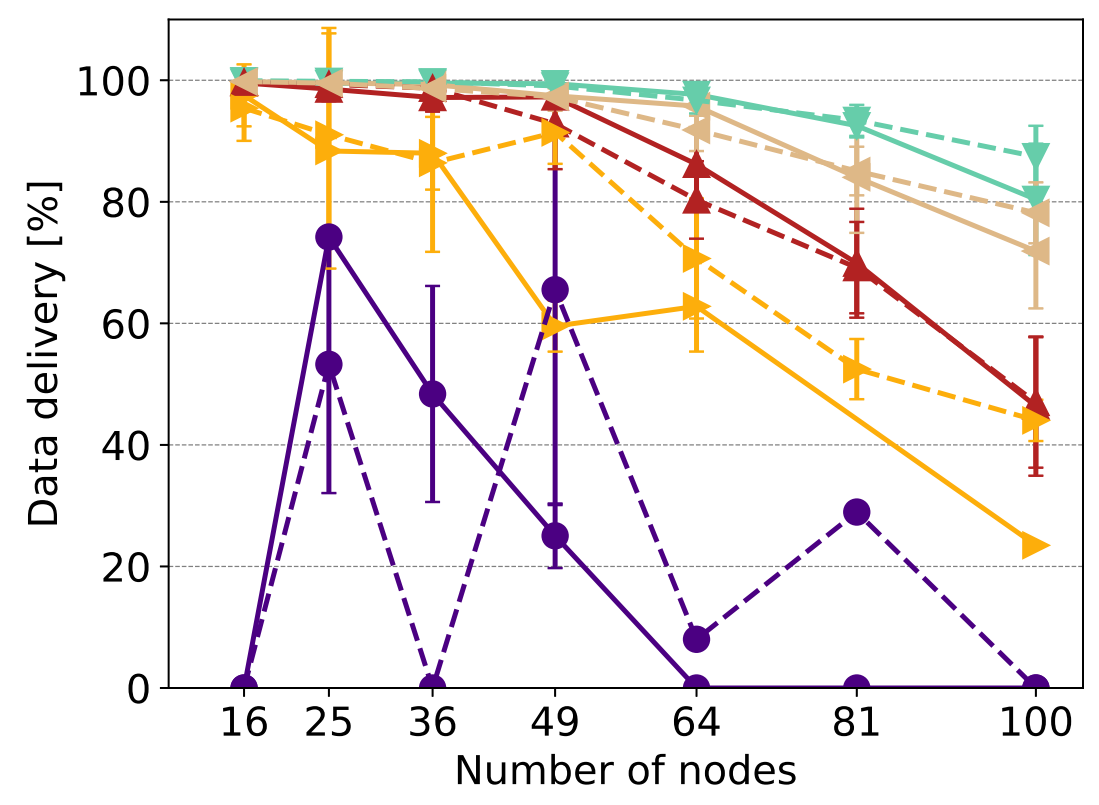

Source: author 
Figure 37 - Duty cycled. Metric: Data delivery. Link setting: Random unidirectional links.

$\begin{array}{ll}- \text { - Collect ND/CD + ContikiMAC (random topology) } & -\mathbf{-} \text { Collect ND/CD + ContikiMAC (grid topology) } \\ - \text { Enhanced ND/CD + ContikiMAC (random topology) } & - \text { - Enhanced ND/CD + ContikiMAC (grid topology) } \\ - \text { Enhanced ND/CD + our RDC -- to nodes (random topology) } & -\mathrm{-} \text { - Enhanced ND/CD + our RDC -- to nodes (grid topology) } \\ - \text { Enhanced ND/CD + our RDC -- to controller (random topology) } & - \text { - Enhanced ND/CD + our RDC -- to controller (grid topology) } \\ - \text { Naive ND/CD + our RDC -- to nodes (random topology) } & - \text { - Naive ND/CD + our RDC -- to nodes (grid topology) }\end{array}$

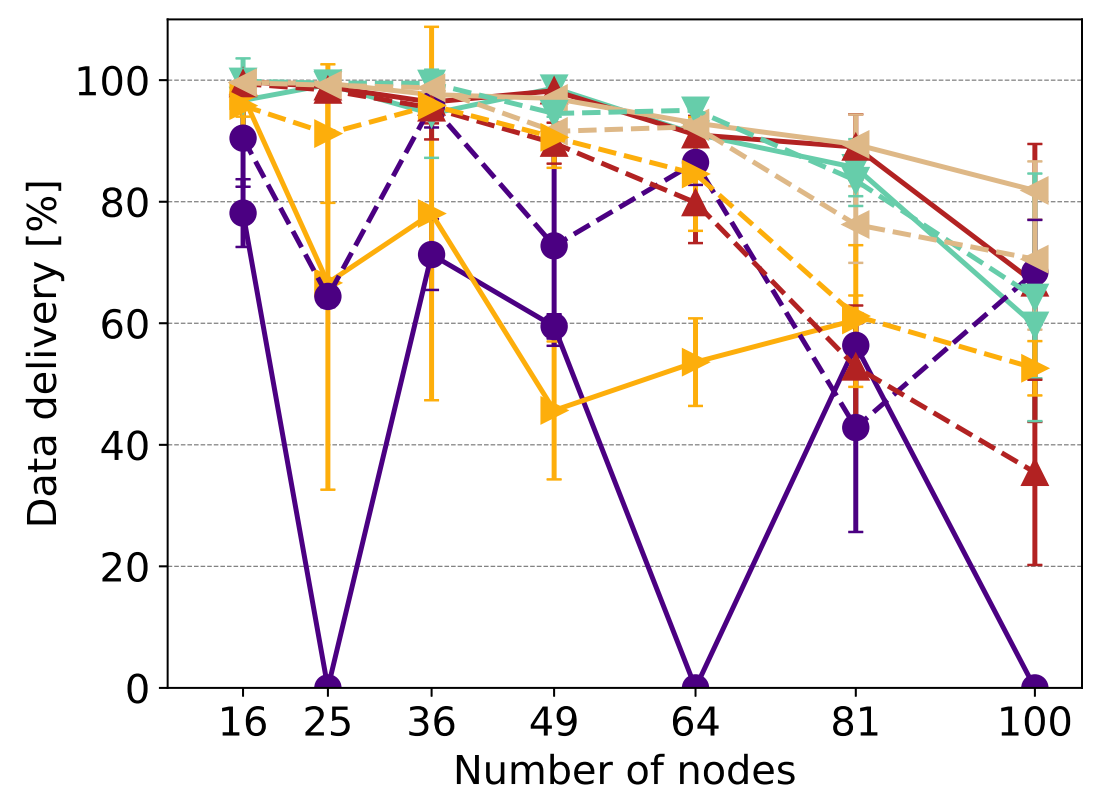

Source: author

Data delivery results are displayed in Figures 34, 35, 36, and 37. The bidirectional link topology (Figure 34) is useful to investigate the effect of the extra load needed to support unidirectional links in comparison to solutions that do not support them. We observed that, in some of the simulation runs for large networks, some of the links were initially detected as unidirectional, since the inbound and outbound components of a bidirectional link are informed separately to the controller. As a consequence, when receivers are configured to send phase information directly to the sender, the controller sets and maintains control routes for communicating phase information. This extra overhead degrades performance.

The addition of a link from the controller to the other nodes (Figure 35) causes the Collect-based discovery protocol to fail, since it cannot correctly handle long-distance unidirectional links. In this scenario, there is no statistical difference between using the enhanced algorithm with ContikiMAC and using it with our RDC sending phase information to the controller, except in the case of large grid networks, wherein the send-to-nodes version of our RDC presented lower data yield.

Device heterogeneity causes some nodes to reach further than others, but the RDC does not influence the delivery rate in small networks (Figure 36). In large networks, the overhead of maintaining the phase information hinders rather than helps, as we observe a degradation in performance. For this type of topology, using a unidirectional link typically 
reduces the path length by only one hop, which is small compared to the several hops reduction expected in the "controller to all" topology.

The "send to controller" version of our RDC yields small gains $(\approx 3 \%)$ in the random unidirectional links with random topology scenario (Figure 37), although the results are statistically different only for the 100-node network. The algorithm version that sends phase information directly to nodes tends to perform worse, presenting a statistically inferior data yield for the grid topology. The unidirectional links enable a larger number of possible routes in the network, alleviating link-layer congestion bottlenecks. This effect is more pronounced in the random network topology, since it contains more bottleneck nodes and links.

The general trend for the simple ND/CD is similar to the one observed on the non-RDC experiments: although data yield is relatively high for small networks, it tends to decrease as the network increases. Collect-based ND/CD once again showed its inability to properly handle unidirectional links, often presenting delivery under $80 \%$, or even $0 \%$.

Figure 38 - Duty cycled. Metric: Link discovery rate. Link setting: Bidirectional links only.
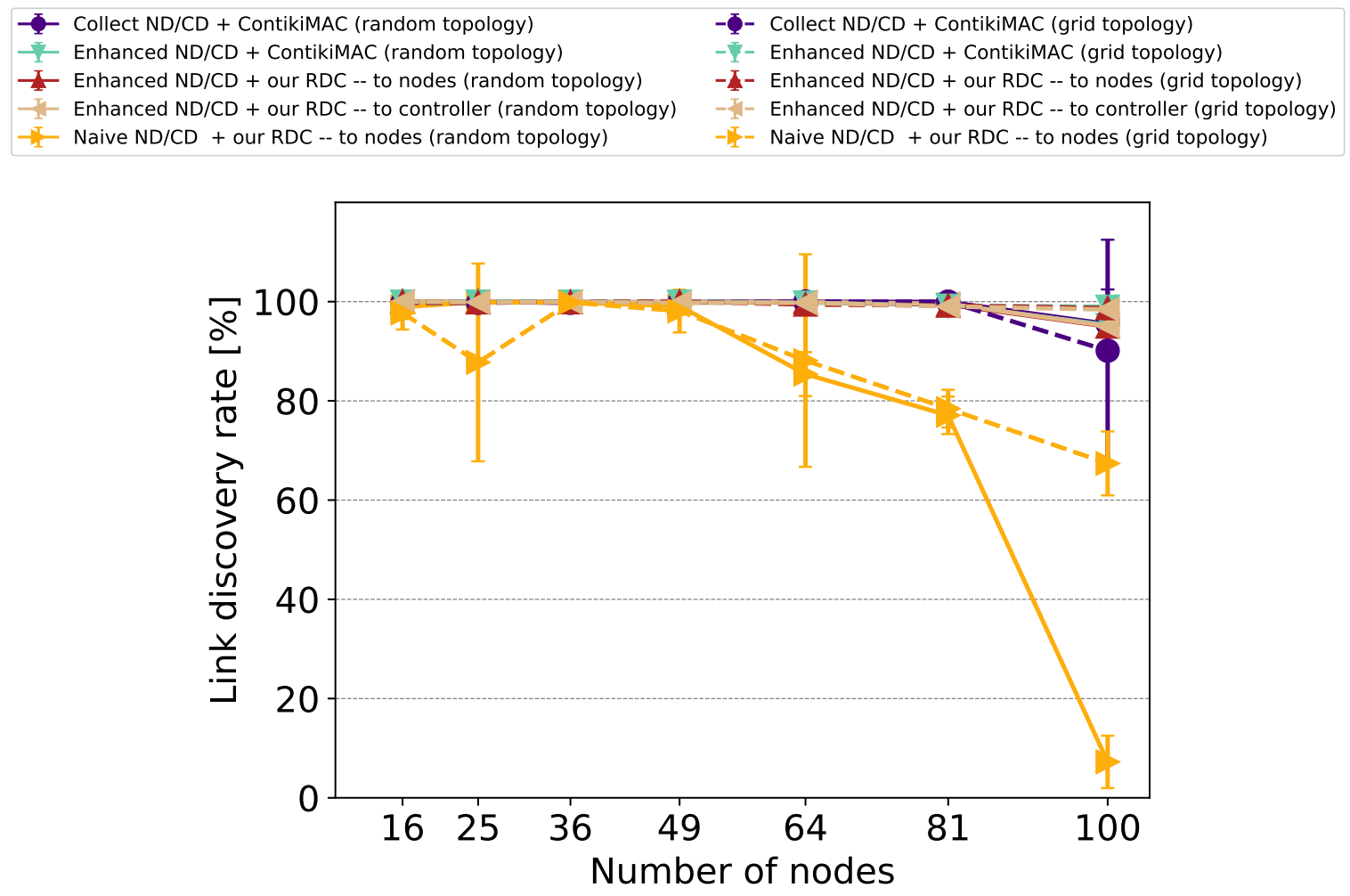

Source: author

Figures 38, 39, 40, and 41 show link discovery rate results. The enhanced discovery protocols presented consistent behavior in terms of detecting all links, regardless of the unidirectional link type. Eventually, links go undetected if the number of links exceeds the neighbor table capacity, which is caused by memory scarceness in network nodes. There was not significant difference between the versions of our RDC and ContikiMAC. 
Figure 39 - Duty cycled. Metric: Link discovery rate. Link setting: Controller to all.

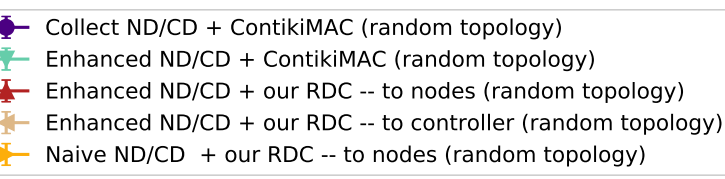

- Collect ND/CD + ContikiMAC (grid topology)

- Enhanced ND/CD + ContikiMAC (grid topology)

- Enhanced ND/CD + our RDC -- to nodes (grid topology)

- Enhanced ND/CD + our RDC -- to controller (grid topology)

- Naive ND/CD + our RDC -- to nodes (grid topology)

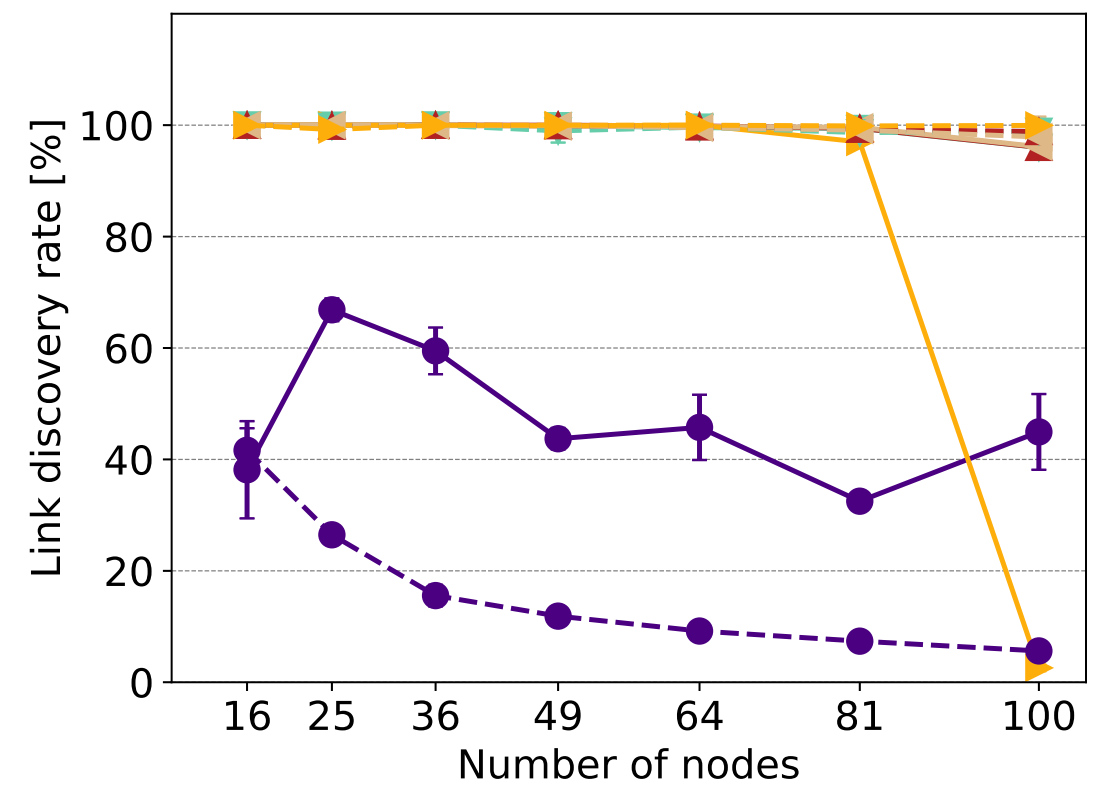

Source: author

Figure 40 - Duty cycled. Metric: Link discovery rate. Link setting: Nodes with increased range.

Collect ND/CD + ContikiMAC (random topology)

Enhanced ND/CD + ContikiMAC (random topology)

Enhanced ND/CD + our RDC -- to nodes (random topology)

Enhanced ND/CD + our RDC -- to controller (random topology)

Naive ND/CD + our RDC -- to nodes (random topology)
- Collect ND/CD + ContikiMAC (grid topology)

- Enhanced ND/CD + ContikiMAC (grid topology)

- I- Enhanced ND/CD + our RDC -- to nodes (grid topology)

- Enhanced ND/CD + our RDC -- to controller (grid topology)

- Naive ND/CD + our RDC -- to nodes (grid topology)

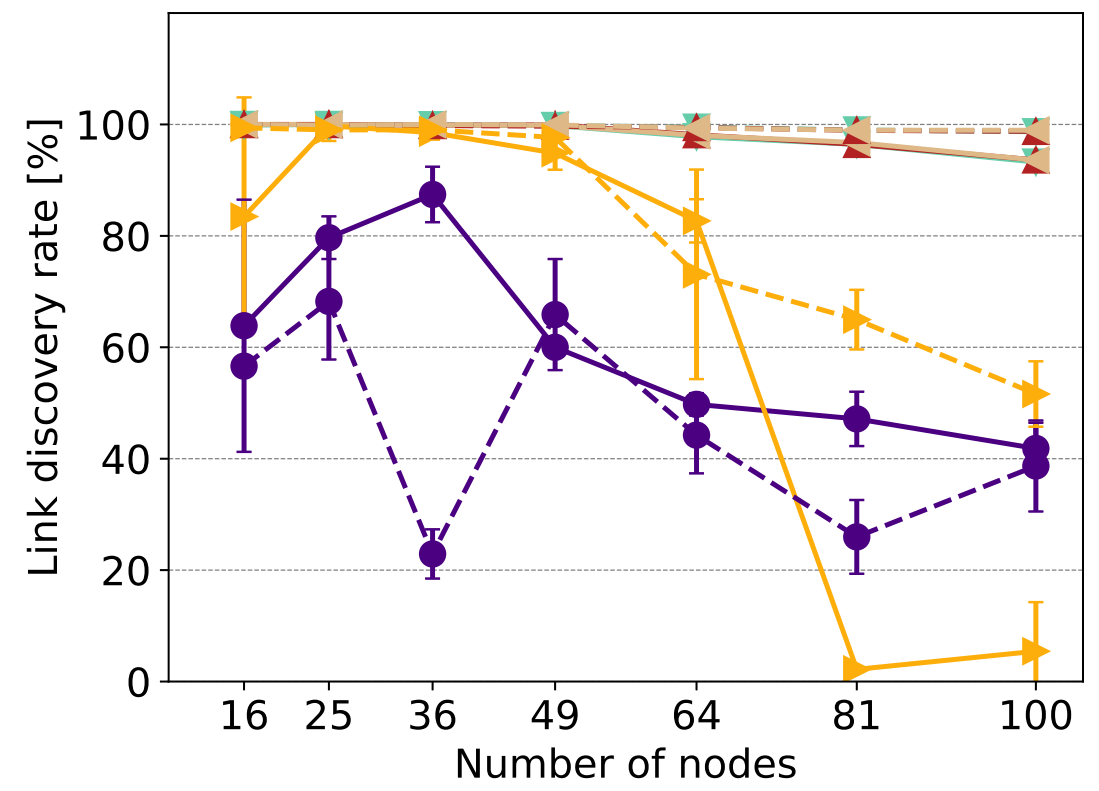

Source: author 
Figure 41 - Duty cycled. Metric: Link discovery rate. Link setting: Random unidirectional links.

\begin{tabular}{|ll}
- - Collect ND/CD + ContikiMAC (random topology) & $-\Phi-$ Collect ND/CD + ContikiMAC (grid topology) \\
- Enhanced ND/CD + ContikiMAC (random topology) & - - Enhanced ND/CD + ContikiMAC (grid topology) \\
- Enhanced ND/CD + our RDC -- to nodes (random topology) & - - Enhanced ND/CD + our RDC -- to nodes (grid topology) \\
- Enhanced ND/CD + our RDC -- to controller (random topology) & - - Enhanced ND/CD + our RDC -- to controller (grid topology) \\
\hline- Naive ND/CD + our RDC -- to nodes (random topology) & - - Naive ND/CD + our RDC -- to nodes (grid topology)
\end{tabular}

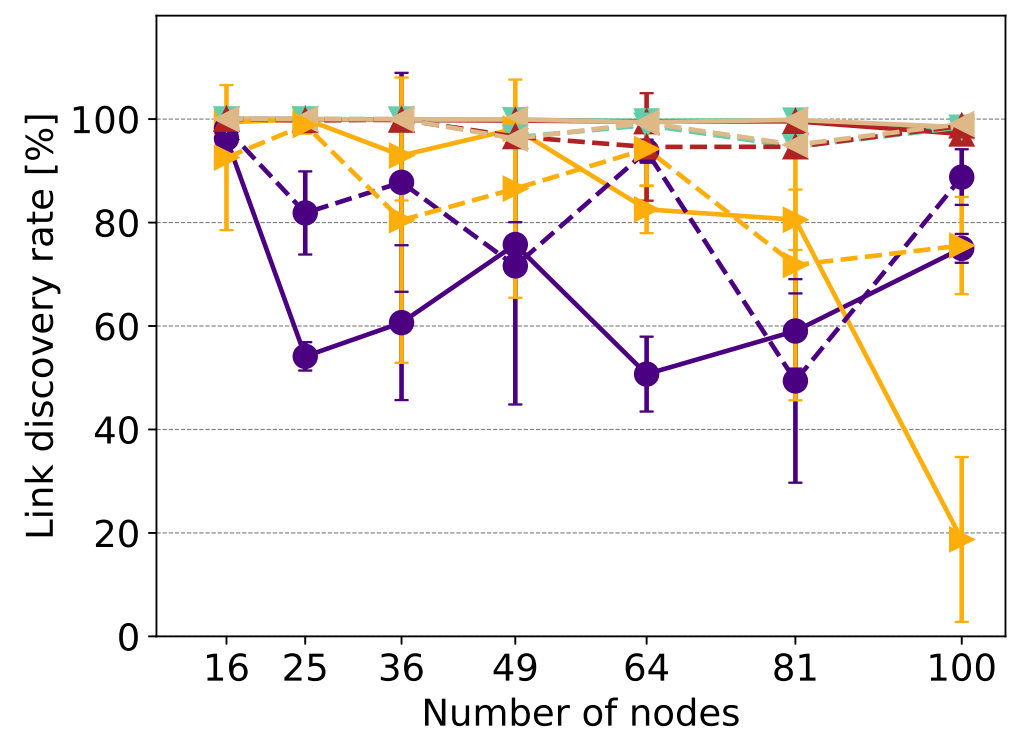

Source: author

The simple discovery algorithm presented high discovery rates for most link configurations up to 36-node networks. It performed worse on the node heterogeneity scenario (Figure 40), since it is the configuration with the highest density of links. It performed well on Figure 39, the controller-to-all topology, as the controller has easy access to all nodes in the network, while the reduced transmission rate, introduced by RDC usage, avoids memory overflow issues occurred on non-RDC simulations.

Regarding the Collect-based discovery protocol, the link discovery rate is greatly impacted by unidirectional links. This discovery protocol may mistakenly assume that a unidirectional links is bidirectional, causing heavy losses in the network. Nodes far from the controller might not be able to join the network, and, therefore, do not report their neighborhood status.

It is noteworthy that delivery rate is correlated to discovery rate, with a Pearson correlation coefficient of 0.88 . As the percentage of discovered links decreases, more links becomes bottlenecks and/or nodes farther from the controller are unable to deliver data.

The most noticeable result for the data delay metric is observed with the "controller to all" topology (Figure 43). Using the enhanced algorithm as reference, using long unidirectional links allows for faster initial route setup, decreasing the overall data delivery delay. The maximal improvement is $54.9 \%$, for both grid and random topologies, although there is enough statistical evidence for only 49 and 81-node networks. 
Figure 42 - Duty cycled. Metric: Data delay. Link setting: Bidirectional links only.

- Collect ND/CD + ContikiMAC (random topology)

Enhanced ND/CD + ContikiMAC (random topology)

Enhanced ND/CD + our RDC -- to nodes (random topology)

Enhanced ND/CD + our RDC -- to controller (random topology)

Naive ND/CD + our RDC -- to nodes (random topology)
9- Collect ND/CD + ContikiMAC (grid topology)

- Enhanced ND/CD + ContikiMAC (grid topology)

- Enhanced ND/CD + our RDC -- to nodes (grid topology)

- Enhanced ND/CD + our RDC -- to controller (grid topology)

- Naive ND/CD + our RDC -- to nodes (grid topology)

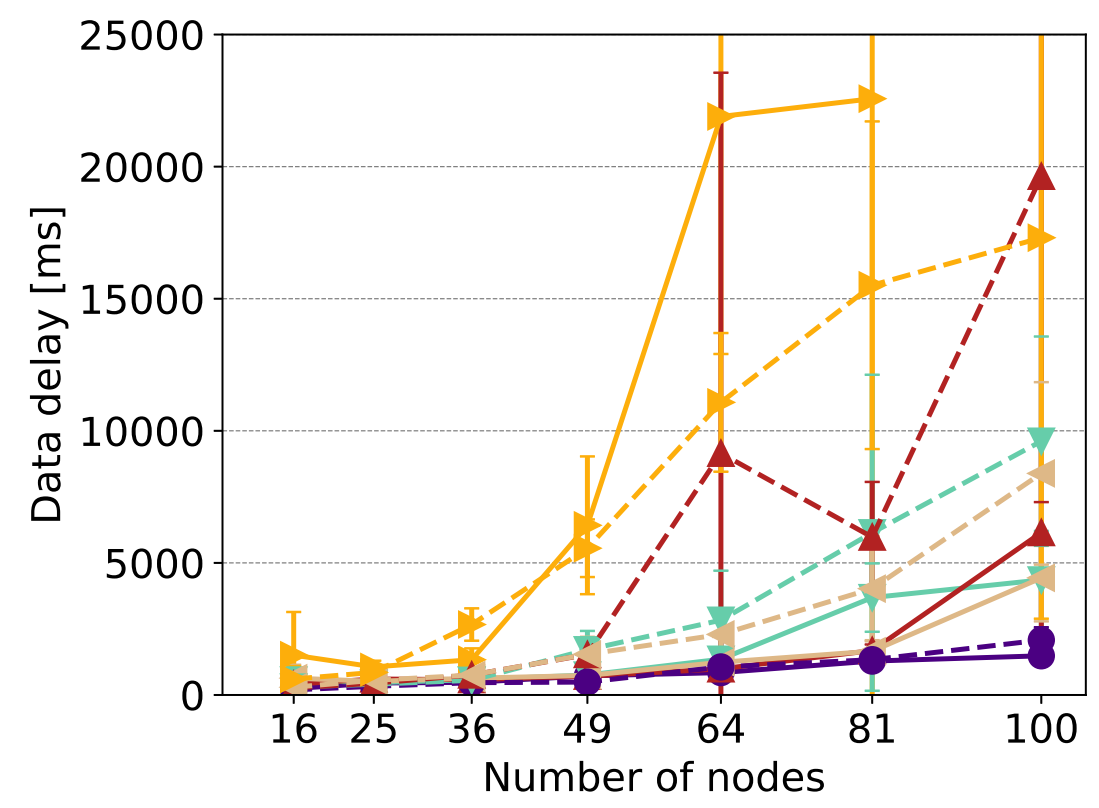

Source: author

Figure 43 - Duty cycled. Metric: Data delay. Link setting: Controller to all.
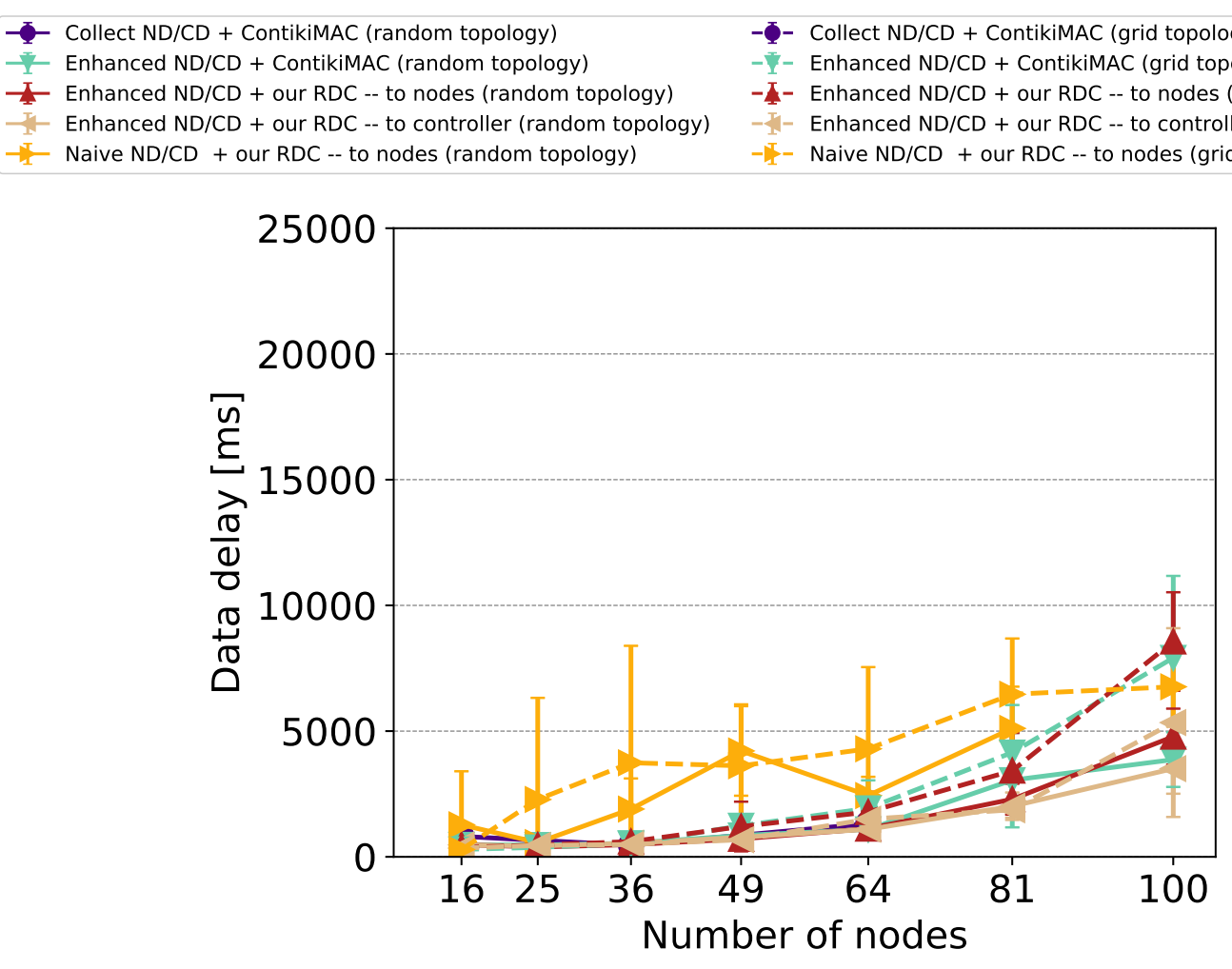

Source: author 
Figure 44 - Duty cycled. Metric: Data delay. Link setting: Nodes with increased range.

- Collect ND/CD + ContikiMAC (random topology)

- Enhanced ND/CD + ContikiMAC (random topology)

Enhanced ND/CD + our RDC -- to nodes (random topology)

Enhanced ND/CD + our RDC -- to controller (random topology)

Naive ND/CD + our RDC -- to nodes (random topology)
- Collect ND/CD + ContikiMAC (grid topology)

- Enhanced ND/CD + ContikiMAC (grid topology)

- Enhanced ND/CD + our RDC -- to nodes (grid topology)

- Enhanced ND/CD + our RDC -- to controller (grid topology)

- Naive ND/CD + our RDC -- to nodes (grid topology)

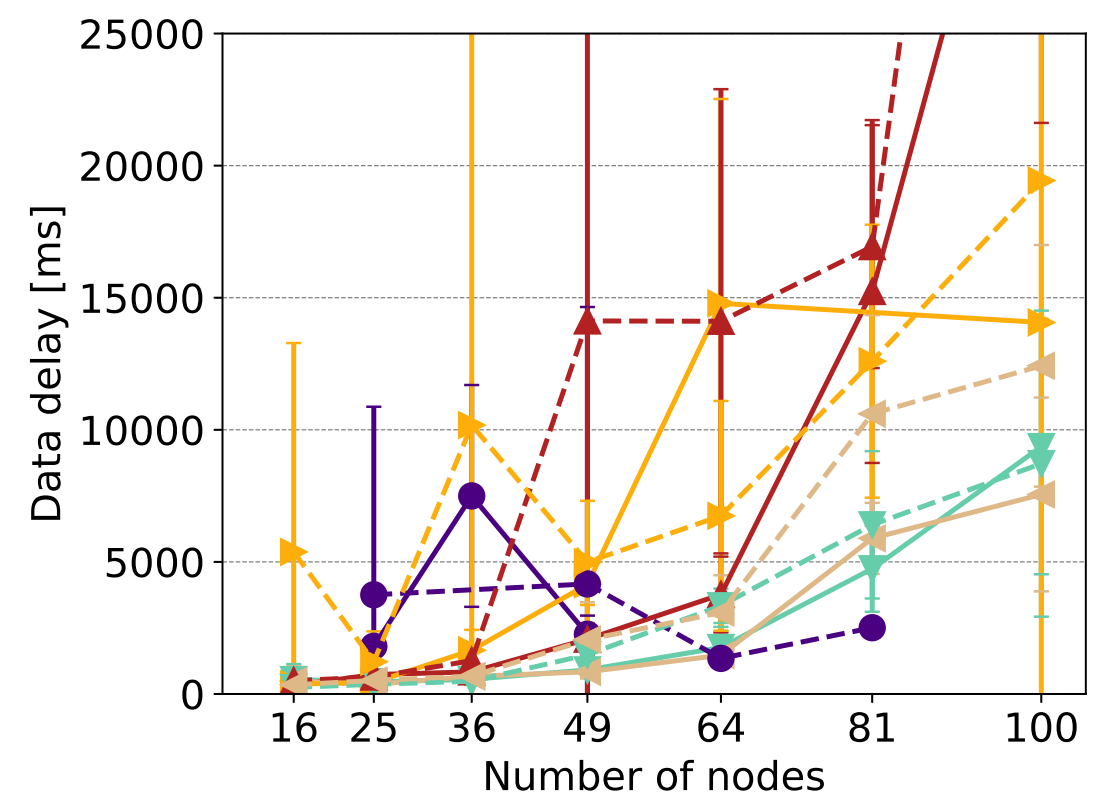

Source: author

Figure 45 - Duty cycled. Metric: Data delay. Link setting: Random unidirectional links.

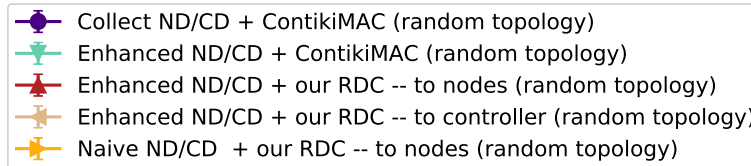

-\$- Collect ND/CD + ContikiMAC (grid topology)

- Enhanced ND/CD + ContikiMAC (grid topology)

- Enhanced ND/CD + our RDC -- to nodes (grid topology)

- Enhanced ND/CD + our RDC -- to controller (grid topology)

- Naive ND/CD + our RDC -- to nodes (grid topology)

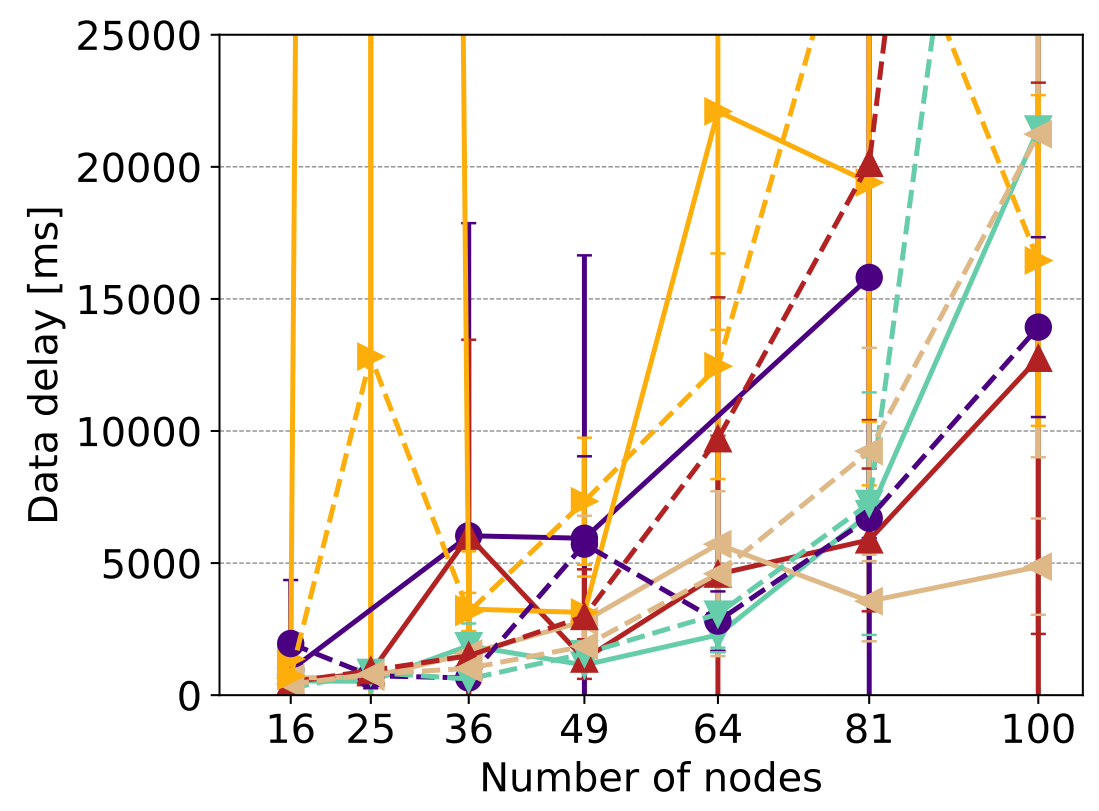

Source: author 
It is not possible to draw conclusions with respect to the other link scenarios (Figures 42, 44, and 45). The issues that cause large confidence intervals explained in Section 6.2.1 are aggravated by the increased medium access contention introduced by the RDCs. The delay metric is sensitive to the initial network setup, meaning that small differences in the packet ordering and collisions at that stage can have great influence on the average delay. Such circumstances account for the large standard deviation values, since some simulations contain packets with a very high delay. This observation is confirmed by comparing the average to the delay median, the latter being significantly smaller in most cases. Nonetheless, there is a trend indicating that, while random topologies present a larger average delay in small networks, they are faster than grids in large networks.

Figure 46 - Duty cycled. Metric: Data delay after convergence. Link setting: Bidirectional links only.

\begin{tabular}{|ll}
- - Collect ND/CD + ContikiMAC (random topology) & - - Collect ND/CD + ContikiMAC (grid topology) \\
- Enhanced ND/CD + ContikiMAC (random topology) & - - Enhanced ND/CD + ContikiMAC (grid topology) \\
- Enhanced ND/CD + our RDC -- to nodes (random topology) & - - Enhanced ND/CD + our RDC -- to nodes (grid topology) \\
- Enhanced ND/CD + our RDC -- to controller (random topology) & - - Enhanced ND/CD + our RDC -- to controller (grid topology) \\
- Naive ND/CD + our RDC -- to nodes (random topology) & - - Naive ND/CD + our RDC -- to nodes (grid topology)
\end{tabular}

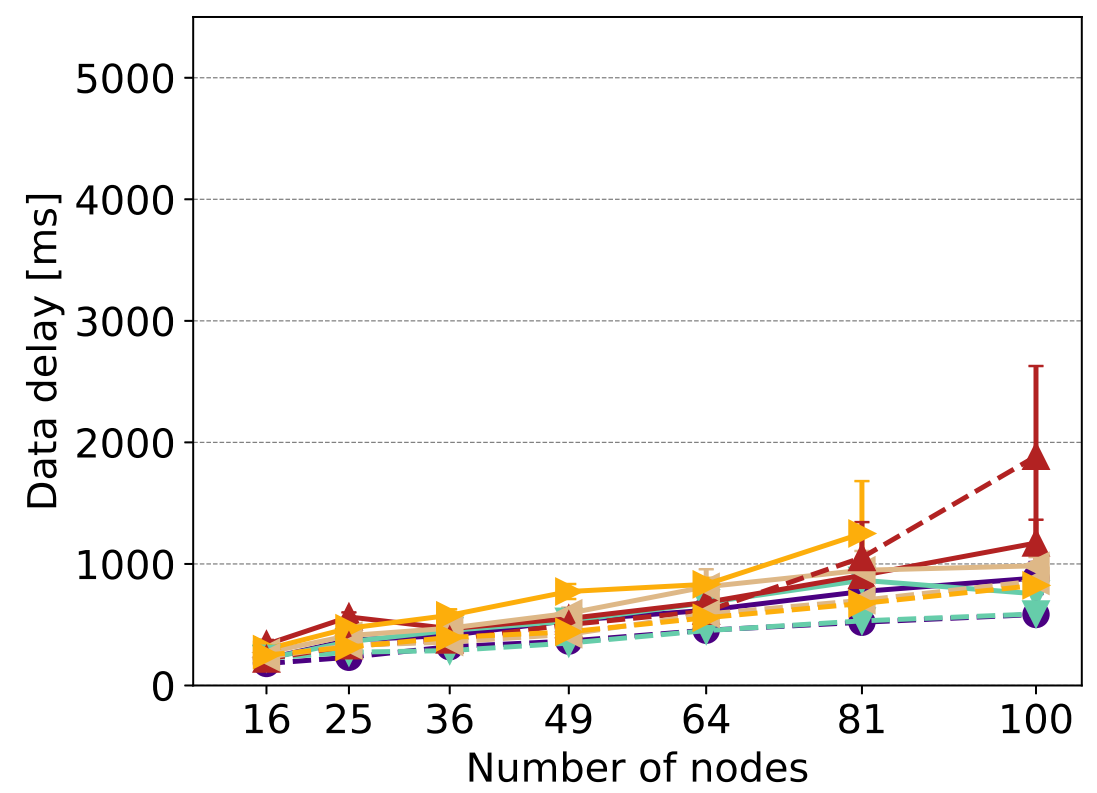

Source: author

To provide better understanding of the delay metric, we analyzed the delay of data packets transmitted after network convergence, shown in Figures 46, 47, 48, and 49. The convergence criterion is the time at which every node has successfully transmitted at least one data packet to the sink.

It is noticeable that the average delay after convergence is significantly smaller than the delay considering all packets, reassuring that the initial flow configuration has a great impact on this metric. 
Figure 47 - Duty cycled. Metric: Data delay after convergence. Link setting: Controller to all.

Collect ND/CD + ContikiMAC (random topology)

Enhanced ND/CD + ContikiMAC (random topology)

Enhanced ND/CD + our RDC -- to nodes (random topology)

Enhanced ND/CD + our RDC -- to controller (random topology)

Naive ND/CD + our RDC -- to nodes (random topology)
Collect ND/CD + ContikiMAC (grid topology)

- Enhanced ND/CD + ContikiMAC (grid topology)

- I- Enhanced ND/CD + our RDC -- to nodes (grid topology)

Enhanced ND/CD + our RDC -- to controller (grid topology)

- Naive ND/CD + our RDC -- to nodes (grid topology)

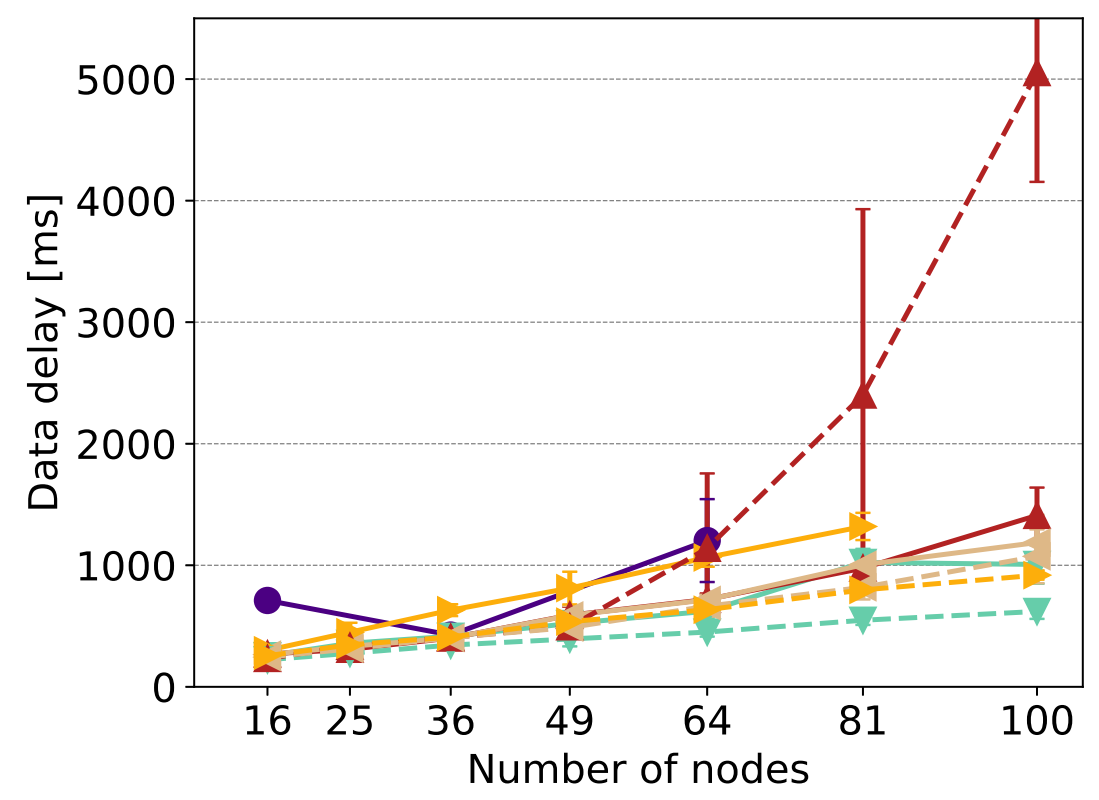

Source: author

Figure 48 - Duty cycled. Metric: Data delay after convergence. Link setting: Nodes with increased range.

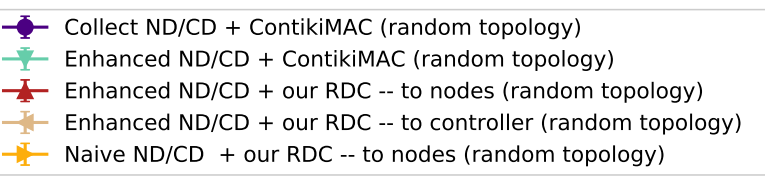
\$- Collect ND/CD + ContikiMAC (grid topology)
- Enhanced ND/CD + ContikiMAC (grid topology)
- $\mathrm{A}$ - Enhanced ND/CD + our RDC -- to nodes (grid topology)
- Enhanced ND/CD + our RDC -- to controller (grid topology)
- Naive ND/CD + our RDC -- to nodes (grid topology)

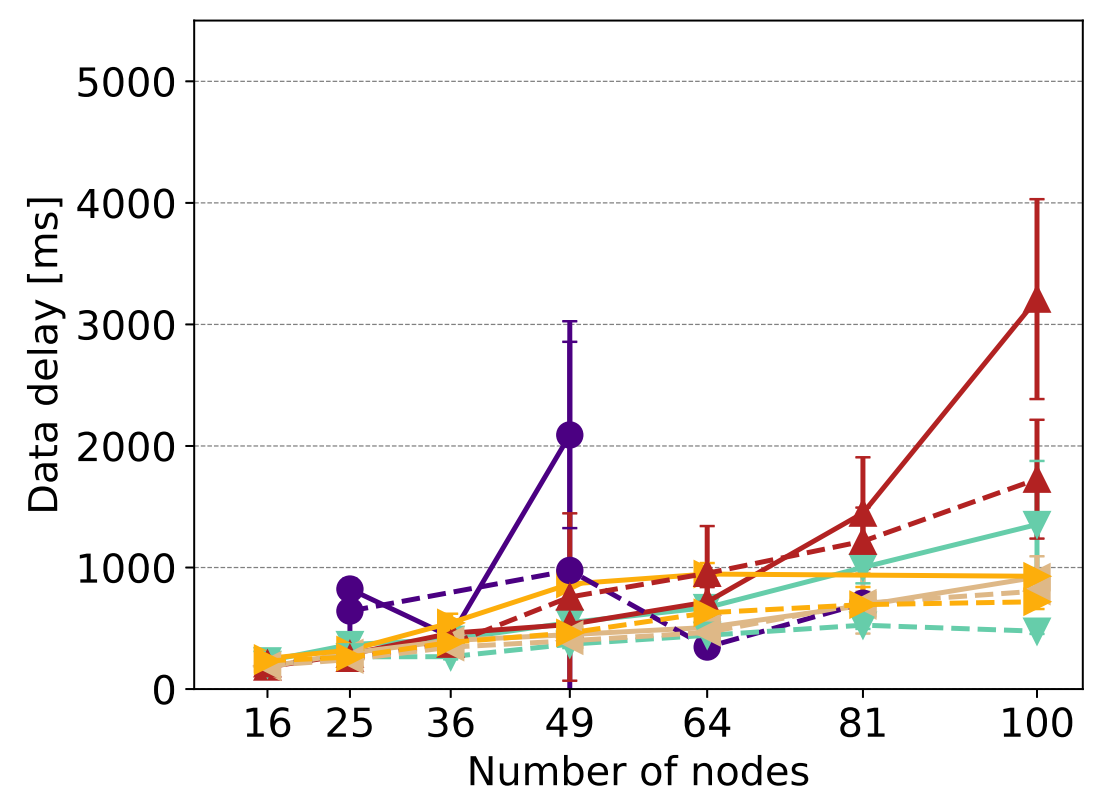

Source: author 
Figure 49 - Duty cycled. Metric: Data delay after convergence. Link setting: Random unidirectional links.
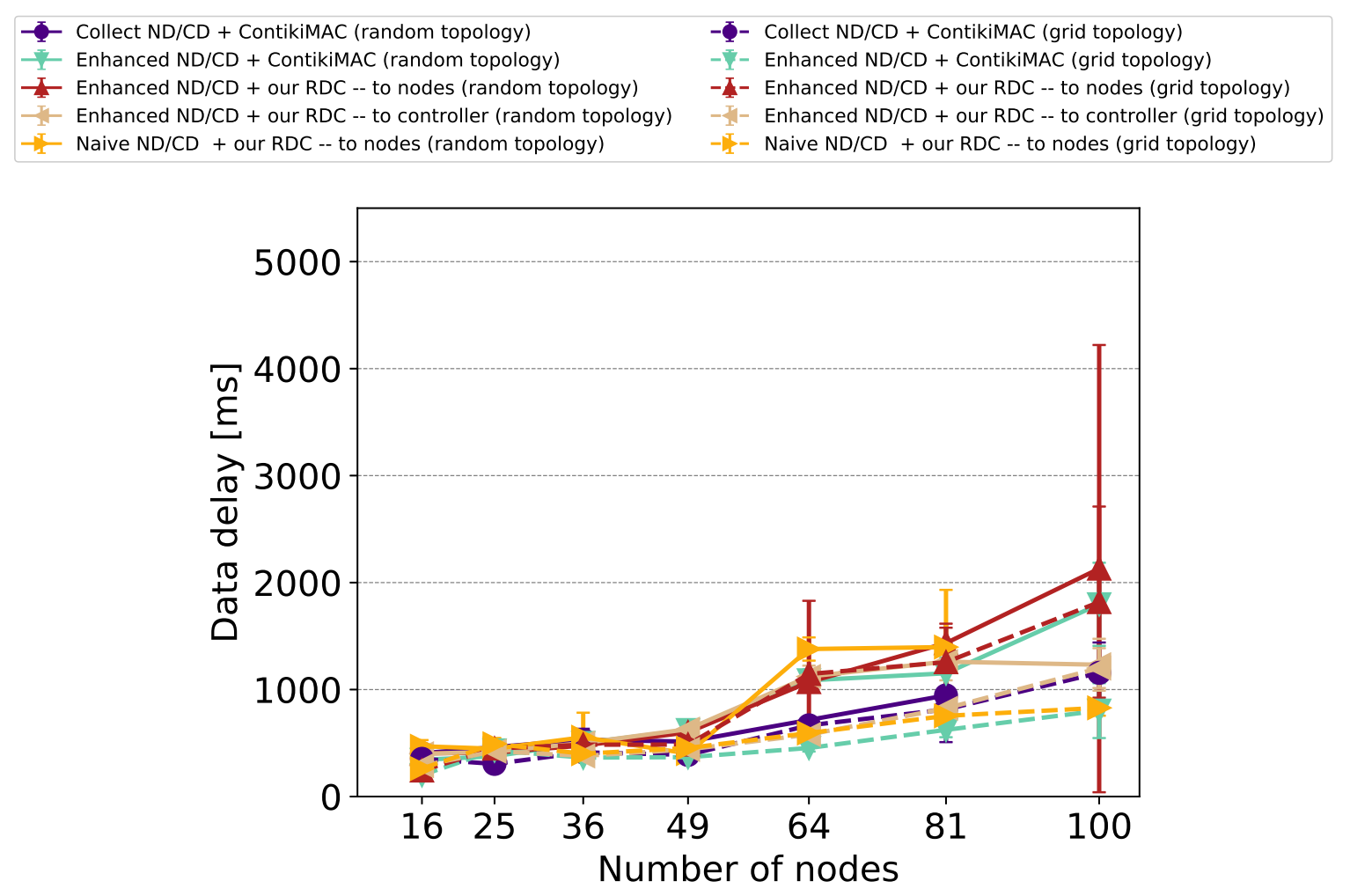

Source: author

Additionally, the confidence intervals are narrower, indicating a consistent steady state behavior. Nonetheless, the enhanced ND/CD algorithm combined with our RDC sending phase information directly to nodes still presents large dispersion in large networks, as some packets are occasionally delayed after many seconds even after convergence. There are two main sources of high delay after convergence: (i) the controller node have enough memory to store several packets in it sending queue, therefore, if a data packet through the controller node, it may be queued for a long time (several seconds) before reaching its destination; (ii) if the phase information used by our RDC is outdated, it may require several attempts before a transmission is successfully completed, increasing packet delay.

The control overhead metric results are shown in Figures 50, 51, 52, and 53. On small and medium networks (up to 64 nodes), the combination of enhanced ND/CD and our RDC (send to controller variation) presents less overhead than the combination this ND/CD and ContikiMAC. The difference reaches 40\% in some scenarios, mainly in the "controller to all" topology. This occurs due to the usage of unidirectional links, which decreases average route size. Shorter routes require fewer flow setup packets and are less prone to link layer errors (reducing retransmissions).

This advantage is less prominent in larger networks (81 and 100), due to the increased number of control messages used to keep the phase information of unidirectional link up-to-date. 
Figure 50 - Duty cycled. Metric: Control overhead. Link setting: Bidirectional links only.

$\begin{array}{ll}- \text { Collect ND/CD + ContikiMAC (random topology) } & -\mathbf{-} \text { Collect ND/CD + ContikiMAC (grid topology) } \\ - \text { Enhanced ND/CD + ContikiMAC (random topology) } & - \text { - Enhanced ND/CD + ContikiMAC (grid topology) } \\ - \text { Enhanced ND/CD + our RDC -- to nodes (random topology) } & - \text { - Enhanced ND/CD + our RDC -- to nodes (grid topology) } \\ - \text { Enhanced ND/CD + our RDC -- to controller (random topology) } & -1-\text { Enhanced ND/CD + our RDC -- to controller (grid topology) } \\ - \text { Naive ND/CD + our RDC -- to nodes (random topology) } & - \text { - Naive ND/CD + our RDC -- to nodes (grid topology) }\end{array}$

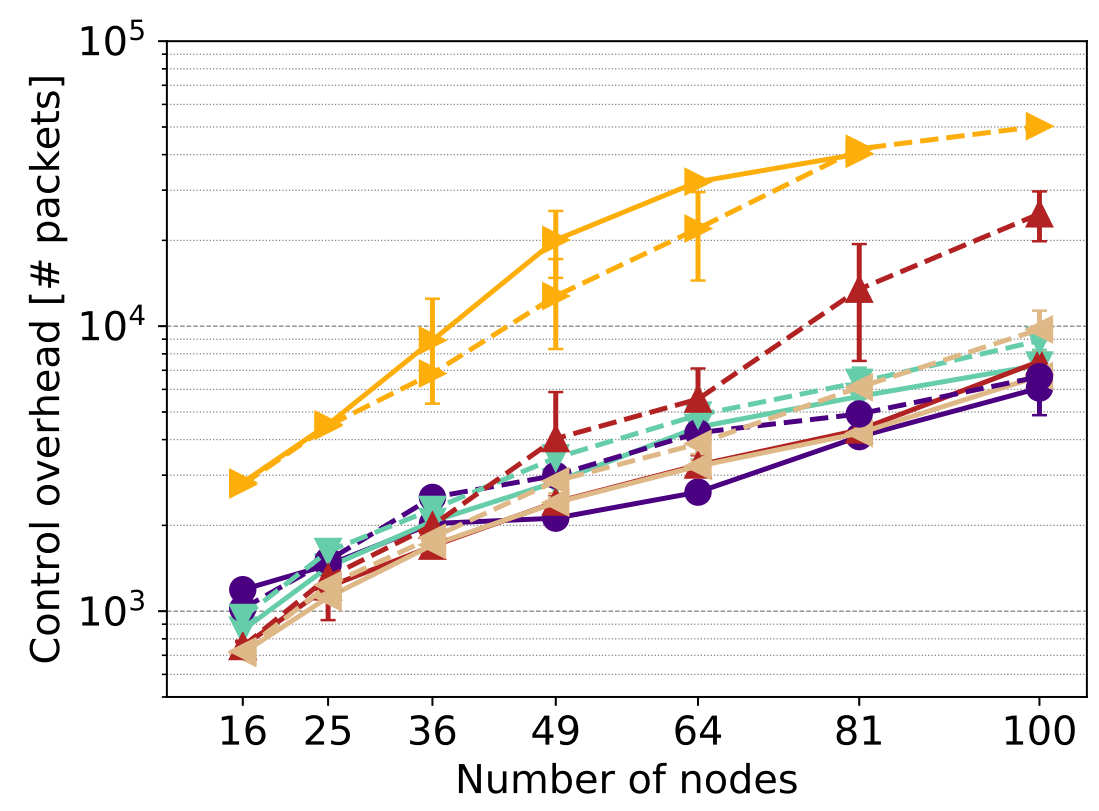

Source: author

Figure 51 - Duty cycled. Metric: Control overhead. Link setting: Controller to all.

$\begin{array}{ll}\text { - Collect ND/CD + ContikiMAC (random topology) } & -\mathbf{-} \text { - Collect ND/CD + ContikiMAC (grid topology) } \\ - \text { Enhanced ND/CD + ContikiMAC (random topology) } & - \text { - Enhanced ND/CD + ContikiMAC (grid topology) } \\ - \text { Enhanced ND/CD + our RDC -- to nodes (random topology) } & - \text { - Enhanced ND/CD + our RDC -- to nodes (grid topology) } \\ - \text { - Enhanced ND/CD + our RDC -- to controller (random topology) } & - \text { - Enhanced ND/CD + our RDC -- to controller (grid topology) } \\ - \text { Naive ND/CD + our RDC -- to nodes (random topology) } & - \text { - Naive ND/CD + our RDC -- to nodes (grid topology) }\end{array}$

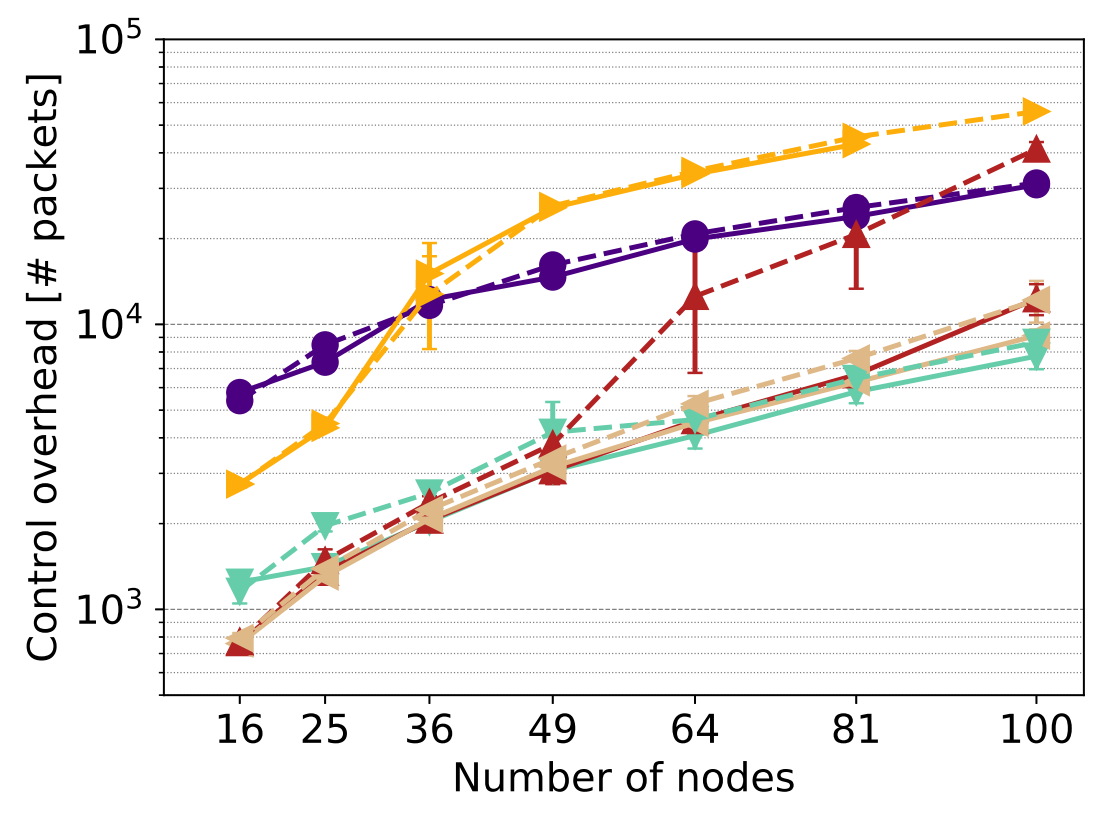

Source: author 
Figure 52 - Duty cycled. Metric: Control overhead. Link setting: Nodes with increased range.

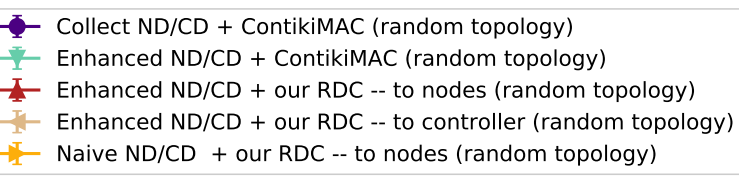

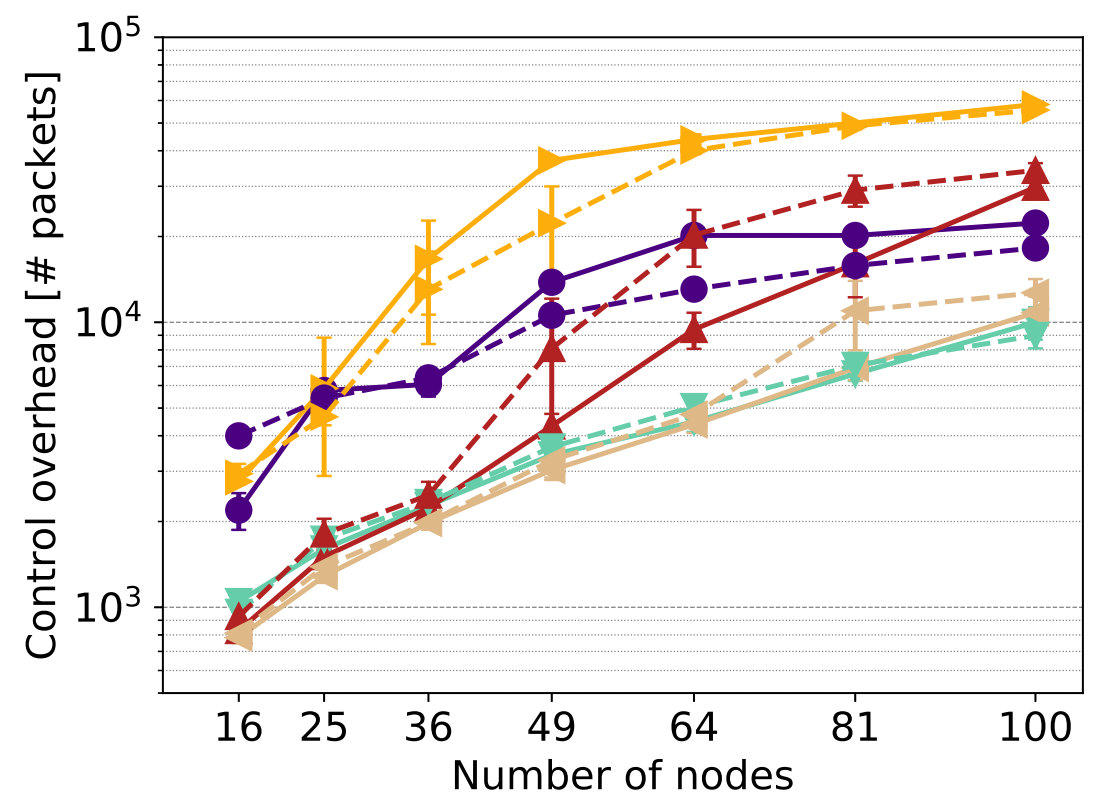

Source: author

Figure 53 - Duty cycled. Metric: Control overhead. Link setting: Random unidirectional links.

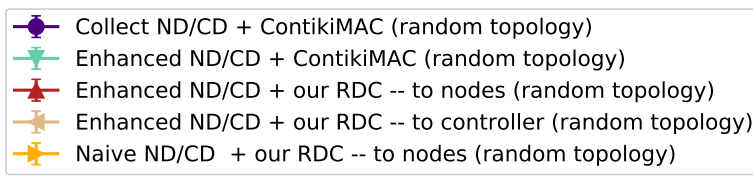

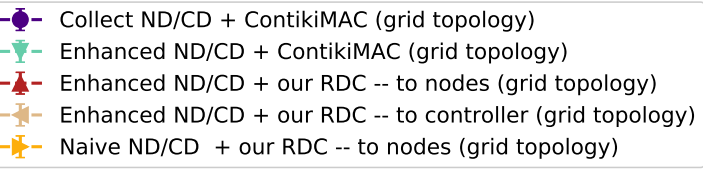


Our RDC variation that sends phase information directly to the nodes does not perform as good as the variation that sends phase information to the controller. Nodes request control routes even for links temporarily detected as unidirectional at network start up. Even if these routes are not further used throughout the experiment, the controller keeps updating the routes according to variations of link quality, increasing the overall overhead. The larger the network, the more pronounced this effect.

The Collect-based approach is only more efficient than the enhanced ND/CD in terms of control overhead in large fully bidirectional topologies. It performs poorly in other link configurations, mostly due to low control packets delivery ratio and consequent retransmissions.

The naive ND/CD, as expected, exhibits the largest control overhead in most scenarios, with the exception of a few cases in which the Collect ND/CD is worse.

Figure 54 - Duty cycled. Metric: Energy usage. Link setting: Bidirectional links only.

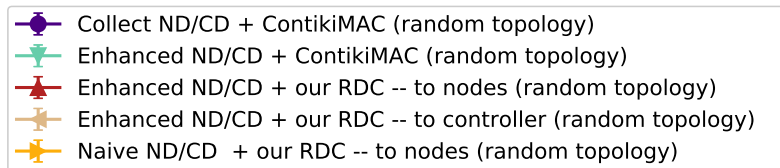

- $\mathbf{-}$ - Collect ND/CD + ContikiMAC (grid topology)

- Enhanced ND/CD + ContikiMAC (grid topology)

- - Enhanced ND/CD + our RDC -- to nodes (grid topology)

- Enhanced ND/CD + our RDC -- to controller (grid topology)

- Naive ND/CD + our RDC -- to nodes (grid topology)

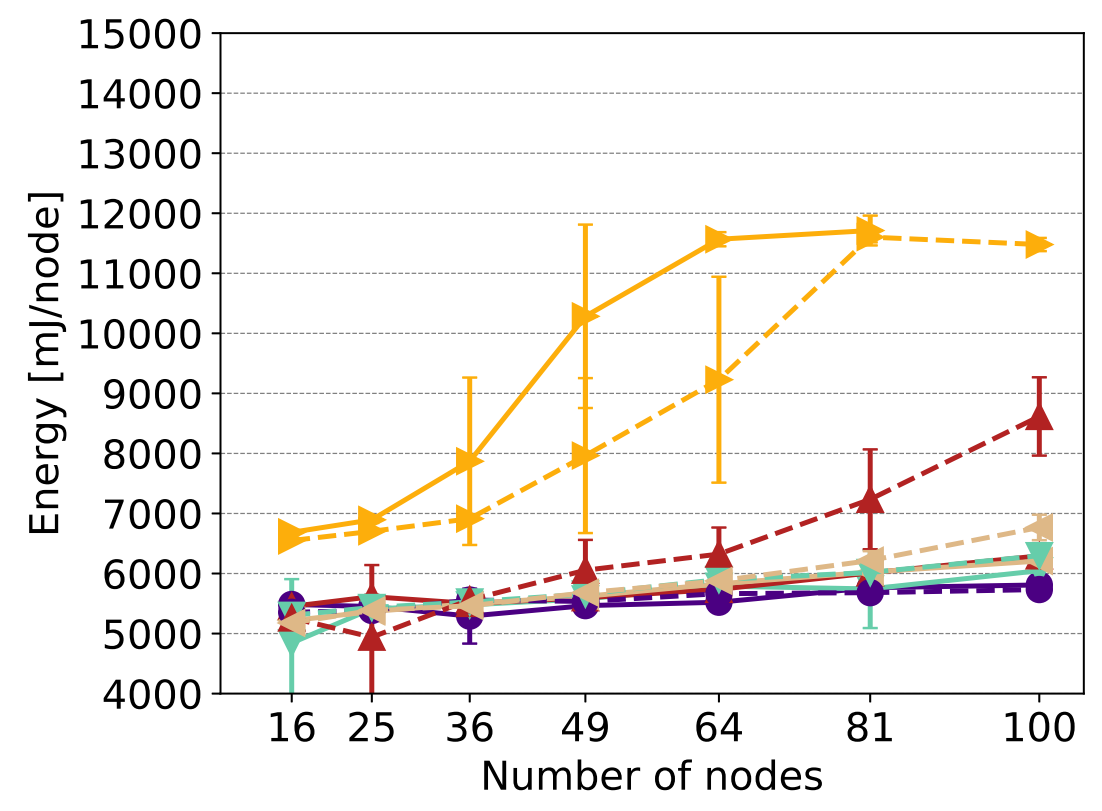

Source: author

We expected that our RDC would reduce overall energy consumption, since using unidirectional links reduces the average path length. However, as observed in Figures 54, 55, 56, and 57, gains from shortening the average length path are ultimately negated by the extra overhead of maintaining control routes and sending phase information.

For most scenarios, there are no significant differences (less than $\pm 5 \%$ ) in energy consumption between the enhanced ND/CD combined with ContikiMAC and the enhanced ND/CD combined with our RDC. Collect-based discovery combined with ContikiMAC 
Figure 55 - Duty cycled. Metric: Energy usage. Link setting: Controller to all.
Collect ND/CD + ContikiMAC (random topology)
Enhanced ND/CD + ContikiMAC (random topology)
Enhanced ND/CD + our RDC -- to nodes (random topology)
Enhanced ND/CD + our RDC -- to controller (random topology)
Naive ND/CD + our RDC -- to nodes (random topology)
- Collect ND/CD + ContikiMAC (grid topology)
- Enhanced ND/CD + ContikiMAC (grid topology)
- Enhanced ND/CD + our RDC -- to nodes (grid topology)
- Enhanced ND/CD + our RDC -- to controller (grid topology)
- Naive ND/CD + our RDC -- to nodes (grid topology)

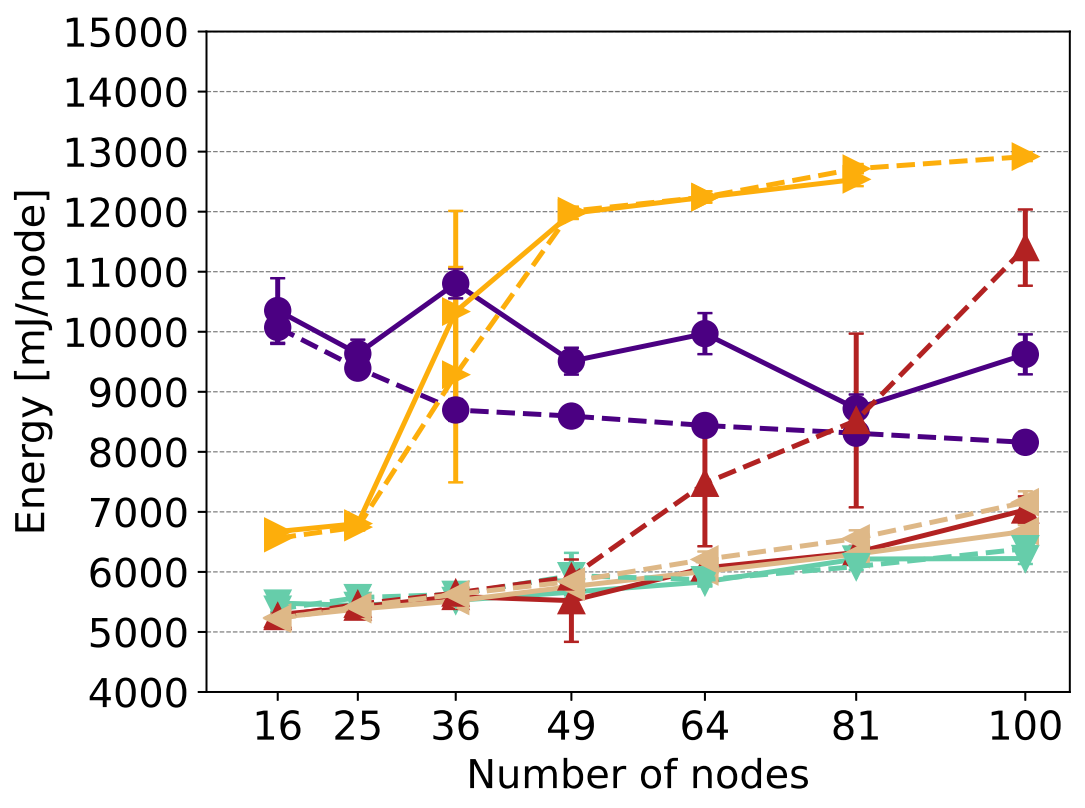

Source: author

Figure 56 - Duty cycled. Metric: Energy usage. Link setting: Nodes with increased range.

\begin{tabular}{|ll}
- - Collect ND/CD + ContikiMAC (random topology) & - - Collect ND/CD + ContikiMAC (grid topology) \\
- Enhanced ND/CD + ContikiMAC (random topology) & - - Enhanced ND/CD + ContikiMAC (grid topology) \\
- Enhanced ND/CD + our RDC -- to nodes (random topology) & - - Enhanced ND/CD + our RDC -- to nodes (grid topology) \\
- Enhanced ND/CD + our RDC -- to controller (random topology) & - - Enhanced ND/CD + our RDC -- to controller (grid topology) \\
- Naive ND/CD + our RDC -- to nodes (random topology) & - - Naive ND/CD + our RDC -- to nodes (grid topology)
\end{tabular}

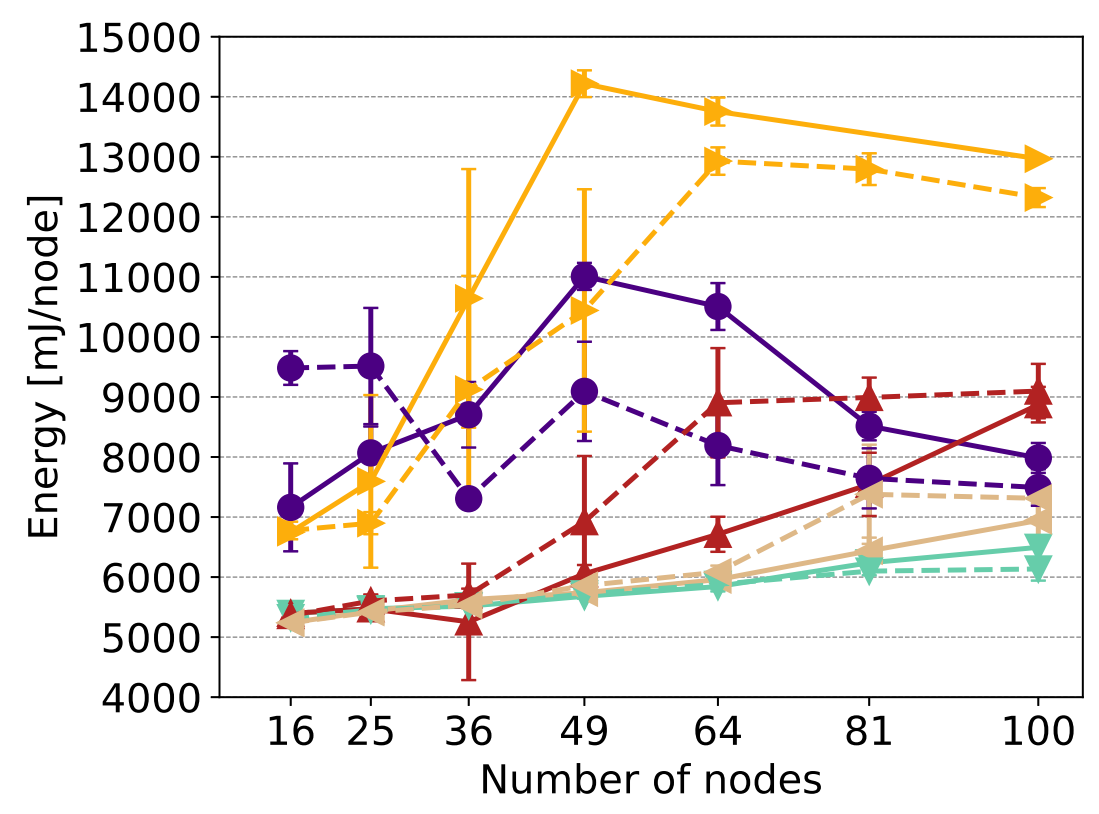

Source: author 
Figure 57 - Duty cycled. Metric: Energy usage. Link setting: Random unidirectional links.
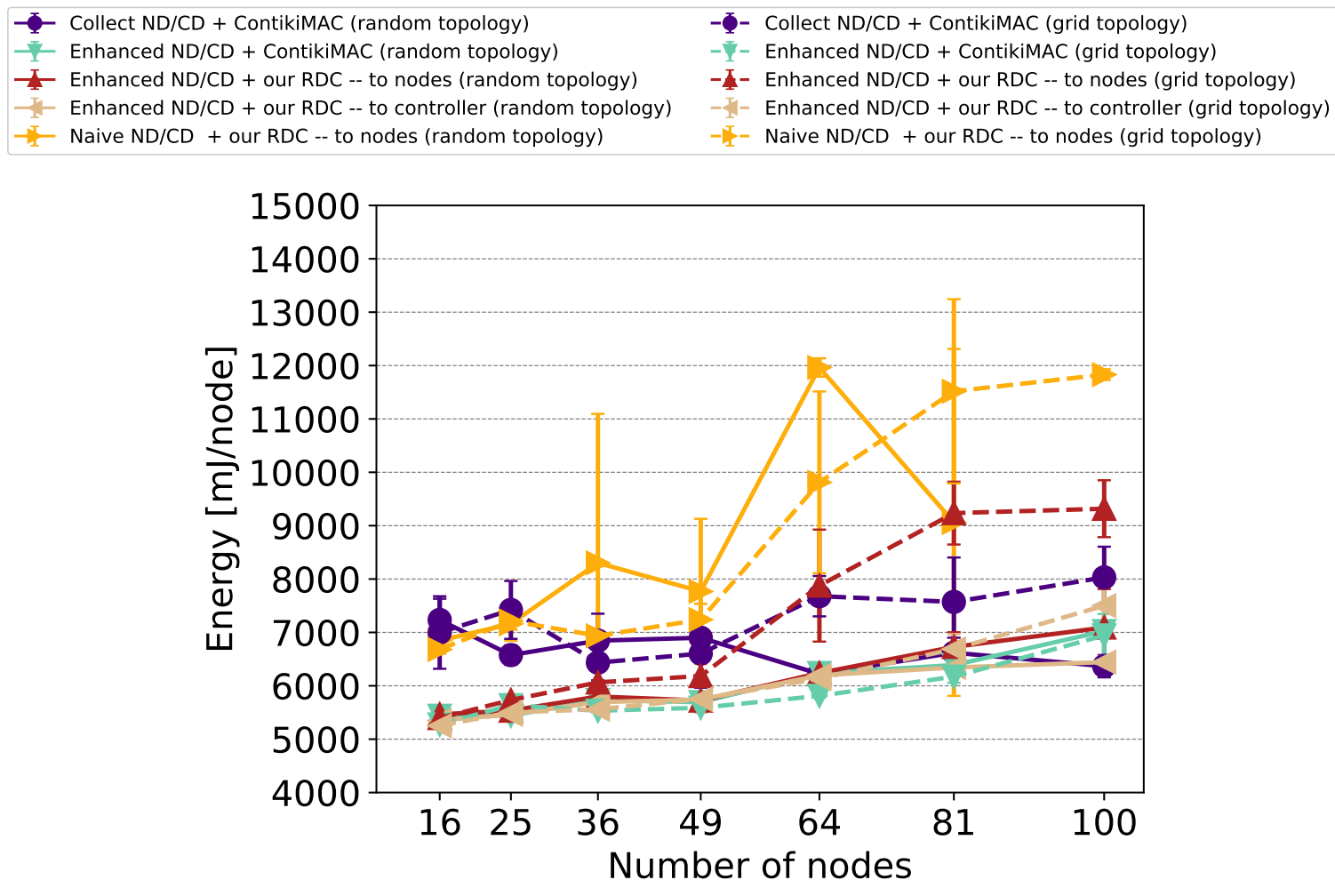

Source: author

also stays in that range when considering the topology with only bidirectional links. In large topologies, the large number of control packets raises the average energy consumption of the Collect-based approach, the naive ND/CD, and the enhanced ND/CD variation that sends phase information directly to the nodes.

\subsection{Conclusions}

At the beginning of this chapter we posed the following question: "What are the gains obtained from exploring unidirectional links in low-power wireless networks?". The results presented previously support the following answers to this question.

When an RDC is not present (Section 6.2.1), using unidirectional links for routing is definitely advantageous. Our enhanced discovery algorithms yield at least $90 \%$ packet delivery, while the same algorithms yielded $85 \%$ delivery on average when unidirectional links were blacklisted; the baseline approach delivered less than $80 \%$ on unidirectional links. Using unidirectional links had a positive effect on the delay metric as well: the average delay was at least halved on $50 \%$ of the test cases, although, using unidirectional links yielded higher delay on $14 \%$ of the test cases.

Prospects are not as positive when an RDC is used (Section 6.2.2). Considering our enhanced discovery algorithms, using unidirectional links with our RDC, more often than 
not $(62.5 \%)$, reduced the delivery rate by a few percentage points. Delay-wise, unidirectional links usage is able to be statistically better only in the presence of long-reaching links; on the other scenarios, although the trend to yield lower delay is also present, the data does not support statistical difference. 


\section{Final remarks}

Unidirectional links emerge naturally on low power wireless networks due to radio transceiver characteristics and environmental factors. Considering the Internet of Things, device heterogeneity is an expected trait that increases the odds of unidirectionality occurrence. Furthermore, the transmission power of non-energy-constrained devices can be purposely increased to save energy from its neighbors. We performed testbed experiments to verify these statements in Chapter 4 .

Therefore, it is essential that routing algorithms are able to detect whether a link is unidirectional or not, since assuming a link is bidirectional could lead to performance degradation or protocol failure. While some approaches blacklist the unidirectional links, we ask the question "What are the gains from exploring unidirectional links in low-power wireless networks?".

We solved the challenge of routing in the presence of unidirectional links by using the Software Defined Networking paradigm. The key idea is leveraging the centralized view of the network available at the controller to facilitate route calculation. To build such centralized view of the network, appropriate discovery algorithms were needed.

First, we provided a proof-concept simple neighbor discovery and controller discovery algorithms (Section 5.1). This set of algorithms demonstrated the potential of using unidirectional links for routing, but, due its simplicity, it has poor scalability. Next, we designed an enhanced version of our discovery algorithms (Section 5.2). Our performance evaluation showed using unidirectional links yields a better performance in terms of packet delivery rate and data delay.

Our last contribution was an asynchronous Radio Duty Cycling algorithm that supports unidirectional links. We tested our discovery algorithms combined with our RDC and combined with ContikiMAC, an state of the art asynchronous RDC. The results revealed that the overhead imposed by our RDC would often negate any gains obtained from exploring the unidirectional links. Minor data delivery gains were observed for shortranged unidirectional links in medium-sized networks. The most noticeable improvement was on the delay metric, when there are long-reaching unidirectional links. A potential future work is investigating the possibility of exploring synchronous RDC protocols to support unidirectional links.

Another future work strand consists on expanding the experiment scenarios. Varying the following parameters could provide further insights into the effect of unidirectional links on WSN performance: (i) vary the percentage of unidirectional links in the random unidirectional links test case; (ii) combine the link test cases (random unidirectional links, 
nodes with increased range, and controller-to-all); (iii) vary the number of data sinks; (iv) stress the network by increasing the data transmission rate and/or increasing the number of nodes; (v) consider composite metrics such as bandwidth-delay product.

At last, since our results show that exploring long-reaching unidirectional links is the most valuable way of exploring unidirectional links, a topic worth investigating, also delegated to future work, is studying how to induce unidirectional links to improve network performance. For example, the controller could be provided with a topology control algorithm to selectively and adaptively select which nodes should have larger or smaller transmission range according to a specified utility function. Such approach could be applicable to clustering and energy management strategies. Similarly, another idea is to explore the use of directional antennas, which may form unidirectional links in the network. If the beam direction is configurable on-the-fly, the network controller could leverage its centralized knowledge to optimally position the antennas.

\subsection{Publications}

Four research papers stemmed directly from the research present is this thesis, listed bellow chronologically:

- "Discovery protocols for sdn-based wireless sensor networks with unidirectional links", presented at the XXXV Brazilian Communications and Signal Processing Symposium (SBrT) (ALVES; MARGI, 2017);

- "No way back? An SDN protocol for directed IoT networks", presented at the 15th Wireless On-demand Network systems and Services Conference (WONS) (ALVES; MARGI; KUIPERS, 2019);

- "Know when to listen: SDN-based protocols for directed IoT networks", published at the Elsevier Computer Communications Journal (ALVES; MARGI; KUIPERS, 2020);

- "Can you hear me? a metric for link asymmetry", published at the Open Journal of Internet Of Things, and presented at the International Workshop on Very Large Internet of Things (ALVES; MARGI, 2020).

Additionally, three other papers, focused on porting the SDN paradigm to WSN, were published during the $\mathrm{PhD}$ in collaboration with research group fellows:

- "IT-SDN: Improved architecture for SDWSN", presented at the XXXV Brazilian Symposium on Computer Networks and Distributed Systems (SBRC) (ALVES et al., 2017); 
- "Software-defined wireless sensor networks approach: Southbound protocol and its performance evaluation", published at the Open Journal of Internet Of Things (MARGI et al., 2018);

- "The cost of software-defining things: A scalability study of software-defined sensor networks", published at the IEEE Access Journal (ALVES et al., 2019).

At last, the paper entitled "Cluster-based Flow Control in Hybrid Software-Defined Wireless Sensor Networks" is under review at the Elsevier Computer Networks Journal. The opportunity to collaborate on this paper arose during the Research Internships Abroad (BEPE - Bolsa Estágio de Pesquisa no Exterior, in Portuguese), under the supervision of Professor Fernando Kuipers. In this paper, we used IT-SDN to provide a proof-of-concept to a system that trades the amount of overhead for the percentage of centrally controlled devices. 


\section{References}

ADAY, L. O.; PASTOR, C. C.; FERNÁNDEZ, A. F. Current Trends of Topology Discovery in OpenFlow-based Software Defined Networks. Castelldefels, 2015. 37

AKKAYA, K.; YOUNIS, M. A survey on routing protocols for wireless sensor networks. Ad Hoc Networks, v. 3, n. 3, p. 325 - 349, 2005. ISSN 1570-8705. Available from Internet: <http://www.sciencedirect.com/science/article/pii/S1570870503000738>. 22

AL-FUQAHA, A. et al. Internet of things: A survey on enabling technologies, protocols, and applications. IEEE Communications Surveys Tutorials, v. 17, n. 4, p. 2347-2376, 2015. 15

ALVES, R. C. A. Redes de sensores sem fio: análise de desempenho da camada de enlace. Dissertação (Mestrado) - Escola Politécnica, Universidade de São Paulo, São Paulo, 2014. 22

ALVES, R. C. A.; MARGI, C. B. Discovery protocols for sdn-based wireless sensor networks with unidirectional links. In: XXXV SIMPÓSIO BRASILEIRO DE TELECOMUNICAÇÕES E PROCESSAMENTO DE SINAIS. São Pedro - SP, Brazil: Sociedade Brasileira de Telecomunicações, 2017. 51, 110

ALVES, R. C. A.; MARGI, C. B. Can you hear me? a metric for link asymmetry. Open Journal of Internet Of Things (OJIOT), RonPub, v. 6, n. 1, p. 82-88, 2020. ISSN 2364-7108. Available from Internet: < https://www.ronpub.com/ojiot/OJIOT_ 2020v6i1n08_Alves.html>. 41, 110

ALVES, R. C. A.; MARGI, C. B.; KUIPERS, F. A. No way back? An SDN protocol for directed IoT networks. In: 15TH WIRELESS ON-DEMAND NETWORK SYSTEMS AND SERVICES CONFERENCE. Wengen, Switzerland: IEEE, 2019. 58, 110

ALVES, R. C. A.; MARGI, C. B.; KUIPERS, F. A. Know when to listen: SDN-based protocols for directed IoT networks. Computer Communications, v. 150, p. 672 - 686, 2020. ISSN 0140-3664. 70, 110

ALVES, R. C. A. et al. IT-SDN: Improved architecture for SDWSN. In: XXXV SIMPÓSIO BRASILEIRO DE REDES DE COMPUTADORES. Belém: Sociedade Brasileira de Computação, 2017. Available at http://www.larc.usp.br/ cbmargi/it-sdn/. 27, 110

ALVES, R. C. A. et al. The cost of software-defining things: A scalability study of software-defined sensor networks. IEEE Access, v. 7, p. 115093-115108, 2019. 27, 29, 39, $76,77,111$

AWERBUCH, B.; BAR-NOY, A.; GOPAL, M. Approximate distributed bellman-ford algorithms. IEEE Transactions on Communications, v. 42, n. 8, p. 2515-2517, Aug 1994. ISSN 0090-6778. 54

BACCOUR, N. et al. F-lqe: A fuzzy link quality estimator for wireless sensor networks. In: SILVA, J. S.; KRISHNAMACHARI, B.; BOAVIDA, F. (Ed.). Wireless Sensor Networks. Berlin, Heidelberg: Springer Berlin Heidelberg, 2010. p. 240-255. ISBN 978-3-642-11917-0. 30 
BACCOUR, N. et al. Reliable link quality estimation in low-power wireless networks and its impact on tree-routing. Ad Hoc Networks, Elsevier Science Publishers B. V., Amsterdam, The Netherlands, The Netherlands, v. 27, n. C, p. 1-25, abr. 2015. ISSN 1570-8705. Available from Internet: <http://dx.doi.org/10.1016/j.adhoc.2014.11.011>. 60

BACCOUR, N. et al. Radio link quality estimation in wireless sensor networks: A survey. ACM Trans. Sen. Netw., Association for Computing Machinery, New York, NY, USA, v. 8, n. 4, set. 2012. ISSN 1550-4859. Available from Internet: $<$ https://doi.org/10.1145/2240116.2240123>. 30

BAI, Y.; CHEN, L. Extended multicast optimized link state routing protocol in manets with asymmetric links. In: GLOBECOM. Washington DC: IEEE, 2007. p. 1312-1317. ISSN 1930-529X. 31

BAKHT, M.; TROWER, M.; KRAVETS, R. H. Searchlight: Won't you be my neighbor? In: PROCEEDINGS OF THE 18TH ANNUAL INTERNATIONAL CONFERENCE ON MOBILE COMPUTING AND NETWORKING. New York, NY, USA: ACM, 2012. (Mobicom '12), p. 185-196. ISBN 978-1-4503-1159-5. Available from Internet: <http://doi.acm.org/10.1145/2348543.2348568>. 35, 36, 38

BARRENETXEA, G. et al. The hitchhiker's guide to successful wireless sensor network deployments. In: PROCEEDINGS OF THE 6TH ACM CONFERENCE ON EMBEDDED NETWORK SENSOR SYSTEMS. New York, NY, USA: ACM, 2008. (SenSys '08), p. 43-56. ISBN 978-1-59593-990-6. Available from Internet: <http://doi.acm.org/10.1145/1460412.1460418>. 59

BINDEL, S.; CHAUMETTE, S.; HILT, B. F-ETX: An Enhancement of ETX Metric for Wireless Mobile Networks. Communication Technologies for Vehicles, v. 9066, p. 117-128, 2015. Available from Internet: < http://link.springer.com/10.1007/978-3-319-17765-6>. 60

BURATTI, C. et al. Testing protocols for the internet of things on the euwin platform. IEEE Internet of Things Journal, v. 3, n. 1, p. 124-133, 2016. 27

CAMPBELL, A. T. et al. A survey of programmable networks. SIGCOMM Comput. Commun. Rev., ACM, New York, NY, USA, v. 29, n. 2, p. 7-23, abr. 1999. ISSN 0146-4833. Available from Internet: <http://doi.acm.org/10.1145/505733.505735>. 26

CHEN, L.; BIAN, K. Neighbor discovery in mobile sensing applications. Ad Hoc Netw., Elsevier Science Publishers B. V., Amsterdam, The Netherlands, The Netherlands, v. 48, n. C, p. 38-52, set. 2016. ISSN 1570-8705. Available from Internet: <http://dx.doi-org.ez67.periodicos.capes.gov.br/10.1016/j.adhoc.2016.05.005>. 36, 37

CHEN, L.; LI, Y.; VASILAKOS, A. V. On oblivious neighbor discovery in distributed wireless networks with directional antennas: Theoretical foundation and algorithm design. IEEE/ACM Transactions on Networking, v. 25, n. 4, p. 1982-1993, Aug 2017. ISSN 1063-6692. 37

CHEN, S. et al. Asynchronous neighbor discovery on duty-cycled mobile devices: Integer and non-integer schedules. In: Proceedings of the 16th ACM International Symposium on Mobile Ad Hoc Networking and Computing. New York, NY, USA: ACM, 2015. (MobiHoc '15), p. 47-56. ISBN 978-1-4503-3489-1. Available from Internet: $<$ http://doi.acm.org/10.1145/2746285.2746297>. 35 
CHEN, X. et al. Performance guaranteed routing protocols for asymmetric sensor networks. IEEE Transactions on Emerging Topics in Computing, v. 1, n. 1, p. 111-120, June 2013. ISSN 2168-6750. 31

COHEN, R.; KAPCHITS, B. Continuous neighbor discovery in asynchronous sensor networks. IEEE/ACM Trans. Netw., IEEE Press, Piscataway, NJ, USA, v. 19, n. 1, p. 69-79, fev. 2011. ISSN 1063-6692. Available from Internet: <http://dx.doi-org.ez67.periodicos.capes.gov.br/10.1109/TNET.2010.2053943>. 36, 38

COUTO, D. S. J. D. et al. A high-throughput path metric for multi-hop wireless routing. Wireless Networks, Springer-Verlag New York, Inc., Secaucus, NJ, USA, v. 11, n. 4, p. 419-434, jul. 2005. ISSN 1022-0038. Available from Internet: <http://dx.doi.org/10.1007/s11276-005-1766-z>. 30, 60

DUNKELS, A. The ContikiMAC Radio Duty Cycling Protocol. Stockholm, Sweden, 2011. Available from Internet: <http://dunkels.com/adam/dunkels11contikimac.pdf $>$. 70

DUNKELS, A.; GRONVALL, B.; VOIGT, T. Contiki - a lightweight and flexible operating system for tiny networked sensors. In: 29TH ANNUAL IEEE INTERNATIONAL CONFERENCE ON LOCAL COMPUTER NETWORKS. Tampa, FL: IEEE, 2004. p. 455-462. 24, 29

DUNKELS, A.; ÖSTERLIND, F.; HE, Z. An adaptive communication architecture for wireless sensor networks. In: PROCEEDINGS OF THE 5TH INTERNATIONAL CONFERENCE ON EMBEDDED NETWORKED SENSOR SYSTEMS. Sydney, Australia: ACM, 2007. p. 335-349. 43

DUNKELS, A. et al. Software-based on-line energy estimation for sensor nodes. In: PROCEEDINGS OF THE 4 TH WORKSHOP ON EMBEDDED NETWORKED SENSORS. Cork Ireland: ACM, 2007. p. 28-32. 80

DUTTA, P.; CULLER, D. Practical asynchronous neighbor discovery and rendezvous for mobile sensing applications. In: Proceedings of the 6th ACM Conference on Embedded Network Sensor Systems. New York, NY, USA: ACM, 2008. (SenSys '08), p. 71-84. ISBN 978-1-59593-990-6. Available from Internet: <http://doi.org/10.1145/1460412.1460420>. $35,36,38$

FERRARI, F. et al. Efficient network flooding and time synchronization with Glossy. In: PROCEEDINGS OF THE 10TH ACM/IEEE INTERNATIONAL CONFERENCE ON INFORMATION PROCESSING IN SENSOR NETWORKS. Chicago, IL: IEEE, 2011. p. 73-84. 23

GALLUCCIO, L. et al. SDN-WISE: Design, prototyping and experimentation of a stateful sdn solution for wireless sensor networks. In: 2015 IEEE CONFERENCE ON COMPUTER COMMUNICATIONS (INFOCOM). Kowloon, Hong Kong: IEEE, 2015. p. 513-521. ISSN 0743-166X. 26, 39

GNAWALI, O. et al. Collection tree protocol. In: . New York, NY, USA: ACM, 2009. (SenSys), p. 1-14. ISBN 978-1-60558-519-2. 31

HALEPLIDIS, E. et al. Software-Defined Networking (SDN): Layers and Architecture Terminology. IETF, 2015. RFC 7426 (Informational). (Request for Comments, 7426). Available from Internet: <http://www.ietf.org/rfc/rfc7426.txt>. 25 
HASAN, M. M. et al. COASYM-MAC: A cooperative asymmetric mac protocol for wireless sensor network. In: 2016 9TH INTERNATIONAL CONFERENCE ON ELECTRICAL AND COMPUTER ENGINEERING (ICECE). Dhaka, Bangladesh: IEEE, 2016. p. 558-561. 32

HEINZELMAN, W. R.; KULIK, J.; BALAKRISHNAN, H. Adaptive protocols for information dissemination in wireless sensor networks. In: PROCEEDINGS OF THE 5TH ANNUAL ACM/IEEE INTERNATIONAL CONFERENCE ON MOBILE COMPUTING AND NETWORKING. [S.l.: s.n.], 1999. p. 174-185. 15

HUI, J.; THUBERT, P. Compression Format for IPv6 Datagrams over IEEE 802.15.4-Based Networks. IETF, 2011. RFC 6282 (Proposed Standard). (Request for Comments, 6282). Available from Internet: <http://www.ietf.org/rfc/rfc6282.txt>. 22

IEEE. 802.15.4-2011 - IEEE Standard for Local and metropolitan area networks-Part 15.4: Low-Rate Wireless Personal Area Networks (LR-WPANs). 2011. 15, 22, 27, 43

IEEE. 802.15.4e-2012 - IEEE Standard for Local and metropolitan area networks-Part 15.4: Low-Rate Wireless Personal Area Networks (LR-WPANs) Amendment 1: MAC sublayer. 2012. 22

INTANAGONWIWAT, C.; GOVINDAN, R.; ESTRIN, D. Directed diffusion: A scalable and robust communication paradigm for sensor networks. In: PROCEEDINGS OF THE 6TH ANNUAL INTERNATIONAL CONFERENCE ON MOBILE COMPUTING AND NETWORKING. New York, NY, USA: Association for Computing Machinery, 2000. (MobiCom '00), p. 56-67. ISBN 1581131976. Available from Internet: <https://doi.org/10.1145/345910.345920>. 16

JAKLLARI, G.; LUO, W.; KRISHNAMURTHY, S. V. An integrated neighbor discovery and mac protocol for ad hoc networks using directional antennas. IEEE Transactions on Wireless Communications, v. 6, n. 3, p. 1114-1024, March 2007. ISSN 1536-1276. 37

JENSEN, G. Kernel probability estimation for binomial and multinomial data. 2015. <https://doi.org/10.7287/peerj.preprints.1156v1>. PeerJ PrePrints 3:e1156v1. 44

JHUMKA, A.; MOTTOLA, L. Neighborhood view consistency in wireless sensor networks. ACM Trans. Sen. Netw., ACM, New York, NY, USA, v. 12, n. 3, p. 19:1-19:41, jul. 2016. ISSN 1550-4859. Available from Internet: <http://doi.org/10.1145/2901296>. 21, 37, 38

JULIEN, C. et al. Blend: Practical continuous neighbor discovery for bluetooth low energy. In: PROCEEDINGS OF THE 16TH ACM/IEEE INTERNATIONAL CONFERENCE ON INFORMATION PROCESSING IN SENSOR NETWORKS. New York, NY, USA: ACM, 2017. (IPSN '17), p. 105-116. ISBN 978-1-4503-4890-4. Available from Internet: <http://doi.org/10.1145/3055031.3055086>. 36, 38

KANDHALU, A.; LAKSHMANAN, K.; RAJKUMAR, R. R. U-connect: A low-latency energy-efficient asynchronous neighbor discovery protocol. In: PROCEEDINGS OF THE 9TH ACM/IEEE INTERNATIONAL CONFERENCE ON INFORMATION PROCESSING IN SENSOR NETWORKS. New York, NY, USA: ACM, 2010. (IPSN '10), p. 350-361. ISBN 978-1-60558-988-6. Available from Internet: < http: //doi.acm.org/10.1145/1791212.1791253>. 35, 36, 38 
KARNAPKE, R.; NOLTE, J. Unidirectional link counter - a routing protocol for wireless sensor networks with many unidirectional links. In: Ad Hoc Networking Workshop (MED-HOC-NET). Vilamoura, Portugal: IEEE, 2015. p. 1-7. 33, 76

KESHAVARZIAN, A. et al. Energy-efficient link assessment in wireless sensor networks. In: IEEE INFOCOM 2004. Hong Kong, China: IEEE, 2004. v. 3, p. 1751-1761 vol.3. ISSN 0743-166X. 36, 38

KIM, H.-S. et al. Reliable and energy-efficient downward packet delivery in asymmetric transmission power-based networks. ACM Trans. Sen. Netw., ACM, New York, NY, USA, v. 12 , n. 4 , p. 34:1-34:25, set. 2016. ISSN 1550-4859. 32

KIM, K.-H.; SHIN, K. G. On accurate measurement of link quality in multi-hop wireless mesh networks. In: PROCEEDINGS OF THE 12TH ANNUAL INTERNATIONAL CONFERENCE ON MOBILE COMPUTING AND NETWORKING. New York, NY, USA: ACM, 2006. (MobiCom '06), p. 38-49. ISBN 1-59593-286-0. Available from Internet: $<$ http://doi.acm.org/10.1145/1161089.1161095>. 60

KINDT, P. H. et al. Griassdi: Mutually assisted slotless neighbor discovery. In: PROCEEDINGS OF THE 16TH ACM/IEEE INTERNATIONAL CONFERENCE ON INFORMATION PROCESSING IN SENSOR NETWORKS. New York, NY, USA: ACM, 2017. (IPSN '17), p. 93-104. ISBN 978-1-4503-4890-4. Available from Internet: <http://doi.acm.org/10.1145/3055031.3055074>. 36, 38

KREUTZ, D. et al. Software-defined networking: A comprehensive survey. Proceedings of the IEEE, v. 103, n. 1, p. 14-76, Jan 2015. ISSN 0018-9219. 24

KUSHALNAGAR, N.; MONTENEGRO, G.; SCHUMACHER, C. IPv6 over Low-Power Wireless Personal Area Networks (6LoWPANs): Overview, Assumptions, Problem Statement, and Goals. Wilmington, DE, 2007. <http://www.rfc-editor.org/rfc/rfc4919. txt>. Available from Internet: <http://www.rfc-editor.org/rfc/rfc4919.txt>. 15

LAI, S.; RAVINDRAN, B.; CHO, H. Heterogenous quorum-based wake-up scheduling in wireless sensor networks. IEEE Transactions on Computers, v. 59, n. 11, p. 1562-1575, Nov 2010. ISSN 0018-9340. 36, 38

LEVIS, P. et al. Tinyos: An operating system for sensor networks. In: Ambient Intelligence. Berlin, Heidelberg: Springer Berlin Heidelberg, 2005. p. 115-148. ISBN 978-3-540-27139-0. Available from Internet: <https://doi.org/10.1007/3-540-27139-2_7>. 24

LUO, T.; TAN, H.-P.; QUEK, T. Q. S. Sensor OpenFlow: Enabling Software-Defined Wireless Sensor Networks. Communications Letters, IEEE, v. 16, n. 11, p. 1896-1899, nov. 2012. Available from Internet: <http://dx.doi.org/10.1109/LCOMM.2012.092812.121712>. 26,37

MAHMUD, A.; RAHMANI, R. Exploitation of openflow in wireless sensor networks. In: INTERNATIONAL CONFERENCE ON COMPUTER SCIENCE AND NETWORK TECHNOLOGY (ICCSNT). Harbin, China: IEEE, 2011. v. 1, p. 594-600. 26, 37

MARGI, C. B. Comunicação, segurança e gerenciamento em redes de sensores sem fio. Tese (Livre Docência em Engenharia de Sistemas Digitais) - Escola Politécnica, Universidade de São Paulo, São Paulo, 2015. 21 
MARGI, C. B. et al. Software-defined wireless sensor networks approach: Southbound protocol and its performance evaluation. Open Journal of Internet Of Things (OJIOT), RonPub, v. 4, n. 1, p. 99-108, 2018. ISSN 2364-7108. Special Issue: PROCEEDINGS OF THE INTERNATIONAL WORKSHOP ON VERY LARGE INTERNET OF THINGS (VLIOT 2018) IN CONJUNCTION WITH THE VLDB 2018 CONFERENCE in Rio de Janeiro, Brazil. Available from Internet: <https://www.ronpub.com/OJIOT_2018v4i1n08_Margi.pdf>. 111

MARGI, C. B.; ALVES, R. C. A.; SEPUlVEDA, J. Sensing as a service: Secure wireless sensor network infrastructure sharing for the internet of things. Open Journal of Internet Of Things (OJIOT), RonPub, v. 3, n. 1, p. 91-102, 2017. ISSN 2364-7108. Special Issue: PROCEEDINGS OF THE INTERNATIONAL WORKSHOP ON VERY LARGE INTERNET OF THINGS (VLIOT 2017) IN CONJUNCTION WITH THE VLDB 2017 CONFERENCE in Munich, Germany. Available from Internet: <http://nbn-resolving.de/urn:nbn:de:101:1-2017080613467>. 26

MCGLYNN, M. J.; BORBASH, S. A. Birthday protocols for low energy deployment and flexible neighbor discovery in ad hoc wireless networks. In: PROCEEDINGS OF THE 2ND ACM INTERNATIONAL SYMPOSIUM ON MOBILE AD HOC NETWORKING \& COMPUTING. New York, NY, USA: ACM, 2001. (MobiHoc '01), p. 137-145. ISBN 1-58113-428-2. Available from Internet: <http://doi.acm.org/10.1145/501431.501435>. 34, 35,38

MCKEOWN, N. et al. Openflow: Enabling innovation in campus networks. SIGCOMM Comput. Commun. Rev., ACM, New York, NY, USA, v. 38, n. 2, p. 69-74, mar. 2008. ISSN 0146-4833. Available from Internet: <http://doi.acm.org/10.1145/1355734.1355746>. 26,37

MEMSIC. TelosB Datasheet. 2011. <http://www.memsic.com/userfiles/files/DataSheets/ WSN/telosb_datasheet.pdf $>$. 27, 79

MENG, T.; WU, F.; CHEN, G. On designing neighbor discovery protocols: A code-based approach. In: IEEE INFOCOM 2014 - IEEE Conference on Computer Communications. Toronto, ON, Canada: IEEE, 2014. p. 1689-1697. ISSN 0743-166X. 36, 38

MILIC, B.; MALEK, M. NPART - Node Placement Algorithm for Realistic Topologies in Wireless Multihop Network Simulation. In: PROCEEDINGS OF THE 2ND INTERNATIONAL CONFERENCE ON SIMULATION TOOLS AND TECHNIQUES. ICST, Brussels, Belgium: ICST (Institute for Computer Sciences, Social-Informatics and Telecommunications Engineering), 2009. (Simutools '09), p. 9:1-9:10. ISBN 978-963-9799-45-5. Software downloadable at <https: //www.informatik.hu-berlin.de/de/Members/milic/NPART $>$. Available from Internet: <https://doi.org/10.4108/ICST.SIMUTOOLS2009.5669>. 79

OLIVEIRA, B. T. de; MARGI, C. B.; GABRIEL, L. B. Tinysdn: Enabling multiple controllers for software-defined wireless sensor networks. In: 2014 IEEE Latin-America Conference on Communications (LATINCOM). Cartagena de Indias, Colombia: IEEE, 2014. p. 1-6. 26, 27, 37, 39

OSTERLIND, F. et al. Cross-level sensor network simulation with COOJA. In: PROCEEDINGS OF THE 31ST IEEE CONFERENCE ON LOCAL COMPUTER NETWORKS. Tampa, FL: IEEE, 2006. ISSN 0742-1303. 78 
PERKINS, C.; BELDING-ROYER, E.; DAS, S. Ad hoc On-Demand Distance Vector (AODV) Routing. IETF, 2003. RFC 3561 (Experimental). (Request for Comments, 3561). Available from Internet: <http://www.ietf.org/rfc/rfc3561.txt>. 31

POLASTRE, J.; HILL, J.; CULLER, D. Versatile low power media access for wireless sensor networks. In: PROCEEDINGS OF THE 2ND INTERNATIONAL CONFERENCE ON EMBEDDED NETWORKED SENSOR SYSTEMS. New York, NY, USA: ACM, 2004. (SenSys '04), p. 95-107. ISBN 1-58113-879-2. Available from Internet: $<$ http://doi.acm.org/10.1145/1031495.1031508>. 23

PUROHIT, A.; PRIYANTHA, B.; LIU, J. Wiflock: Collaborative group discovery and maintenance in mobile sensor networks. In: PROCEEDINGS OF THE 10TH ACM/IEEE INTERNATIONAL CONFERENCE ON INFORMATION PROCESSING IN SENSOR NETWORKS. Chicago, IL, USA: IEEE, 2011. p. 37-48. 35, 38

QIU, Y. et al. Talk more listen less: Energy-efficient neighbor discovery in wireless sensor networks. In: IEEE INFOCOM 2016 - THE 35TH ANNUAL IEEE INTERNATIONAL CONFERENCE ON COMPUTER COMMUNICATIONS. San Francisco, CA, USA: IEEE, 2016. p. 1-9. 35, 36, 38

RAMASUBRAMANIAN, V.; MOSSE, D. Bra: A bidirectional routing abstraction for asymmetric mobile ad hoc networks. IEEE/ACM Transactions on Networking, v. 16, n. 1, p. 116-129, Feb 2008. ISSN 1063-6692. 32, 76

RAYNAL, M. Distributed Algorithms for Message-Passing Systems. Switzerland: Springer Publishing Company, Incorporated, 2013. ISBN 3642381227, 9783642381225. 20, 21, 53, 54

REHMAN, A. U.; AGUIAR, R. L.; BARRACA, J. P. Fault-Tolerance in the Scope of Software-Defined Networking (SDN). IEEE Access, v. 7, p. 124474-124490, 2019. 27

SANG, L.; ARORA, A.; ZHANG, H. On link asymmetry and one-way estimation in wireless sensor networks. ACM Transactions on Sensor Networks, ACM, New York, NY, USA, v. 6, n. 2, p. 12:1-12:25, mar. 2010. ISSN 1550-4859. Available from Internet: <http://doi.acm.org/10.1145/1689239.1689242>. 30, 31, 60

SHIBATA, T. et al. Energy consumption and execution time characterization for the SensorTag IoT platform. In: XXXIV SIMPÓSIO BRASILEIRO DE TELECOMUNICAÇÕES E PROCESSAMENTO DE SINAIS (SBrT 2016). Santarém, Brazil: Sociedade Brasileira de Telecomunicações, 2016. p. 55-59. 70

SRINIVASAN, K. et al. An empirical study of low-power wireless. ACM Trans. Sen. Netw., Association for Computing Machinery, New York, NY, USA, v. 6, n. 2, mar. 2010. ISSN 1550-4859. Available from Internet: < https://doi.org/10.1145/1689239.1689246>. 31

SUN, W. et al. Hello: A generic flexible protocol for neighbor discovery. In: IEEE INFOCOM 2014 - IEEE Conference on Computer Communications. Toronto, ON, Canada: IEEE, 2014. p. 540-548. ISSN 0743-166X. 36, 38

TEXAS INSTRUMENTS. CC2420 2.4 GHz IEEE 802.15.4 / ZigBee-ready RF Transceiver. Dallas, Texas, 2004. (Rev. C). 41, 44, 80

TEXAS INSTRUMENTS. Multi-Standard CC2650 SensorTag Design Guide. Dallas, Texas, 2015. 41 
Theodorou, T.; Mamatas, L. Software defined topology control strategies for the internet of things. In: IEEE CONFERENCE ON NETWORK FUNCTION VIRTUALIZATION AND SOFTWARE DEFINED NETWORKS (NFV-SDN). Berlin, Germany: IEEE, 2017. p. 236-241. 39

VASUDEVAN, S. et al. Neighbor discovery in wireless networks and the coupon collector's problem. In: PROCEEDINGS OF THE 15TH ANNUAL INTERNATIONAL CONFERENCE ON MOBILE COMPUTING AND NETWORKING. New York, NY, USA: ACM, 2009. (MobiCom '09), p. 181-192. ISBN 978-1-60558-702-8. Available from Internet: <http://doi.acm.org/10.1145/1614320.1614341>. 34, 38

WANG, K.; MAO, X.; LIU, Y. Blinddate: A neighbor discovery protocol. In: 2013 42ND INTERNATIONAL CONFERENCE ON PARALLEL PROCESSING. Lyon, France: IEEE, 2013. p. 120-129. ISSN 0190-3918. 36, 38

WEI, L. et al. Lightning: A high-efficient neighbor discovery protocol for low duty cycle wsns. IEEE Communications Letters, v. 20, n. 5, p. 966-969, May 2016. ISSN 1089-7798. 35,38

WINTER, T. et al. RPL: IPv6 Routing Protocol for Low-Power and Lossy Networks. IETF, 2012. RFC 6550 (Proposed Standard). (Request for Comments, 6550). Available from Internet: <http://www.ietf.org/rfc/rfc6550.txt>. 16, 22, 31

WOO, A.; CULLER, D. Evaluation of Efficient Link Reliability Estimators for Low-Power Wireless Networks. Berkeley, 2003. Available from Internet: <http://www2.eecs.berkeley.edu/Pubs/TechRpts/2003/6239.html>. 60

WOO, A.; TONG, T.; CULLER, D. Taming the underlying challenges of reliable multihop routing in sensor networks. In: PROCEEDINGS OF THE 1 ST INTERNATIONAL CONFERENCE ON EMBEDDED NETWORKED SENSOR SYSTEMS. New York, NY, USA: ACM, 2003. (SenSys '03), p. 14-27. ISBN 1-58113-707-9. Available from Internet: <http://doi.acm.org/10.1145/958491.958494>. 59

YILDIRIM, K. S. et al. Ink: Reactive kernel for tiny batteryless sensors. In: PROCEEDINGS OF THE 16TH ACM CONFERENCE ON EMBEDDED NETWORKED SENSOR SYSTEMS. New York, NY, USA: Association for Computing Machinery, 2018. (SenSys '18), p. 41-53. ISBN 9781450359528. Available from Internet: <https://doi.org/10.1145/3274783.3274837>. 24

ZHOU, G. et al. Impact of radio irregularity on wireless sensor networks. In: Proceedings of the 2nd International Conference on Mobile Systems, Applications, and Services. New York, NY, USA: Association for Computing Machinery, 2004. (MobiSys '04), p. 125-138. ISBN 1581137931. Available from Internet: <https://doi.org/10.1145/990064.990081>. 31,41 